\title{
Social Interactions In Non-Monetary \\ Accommodation Sharing: \\ Host's Motivation and Value Co-Creation
}

\author{
By \\ Undhan Sevisari
}

A thesis

submitted to the Victoria University of Wellington in partial fulfilment of the requirements for the degree of Master of Tourism Management

Victoria University of Wellington

2019 


\section{Abstract}

Accommodation sharing, as collaborative consumption platforms, fosters memorable tourism experiences by allowing individuals to have direct interactions with hosts and local communities. Airbnb and CouchSurfing exemplify two of the most popular accommodation-sharing platforms, representing the distinction between monetary and non-monetary platforms. While Airbnb as a monetary accommodation sharing platform has gained much attention in the academic literature, little is known regarding CouchSurfing and its non-monetary model. Current research postulates that by the absence of financial involvement, CouchSurfing offers more intimacy and experience in the host-guest relationship. Meanwhile, shifts in tourist behaviour have determined the direction of value co-creation in the tourism industry. Together with tourism providers and other social actors, tourists are engaged in active participation and interaction to create value jointly and for all participants.

Motivated by the extant literature on accommodation sharing and value cocreation, this research contributes to the conceptualisation of value co-creation in non-monetary accommodation sharing through the perspective of the host. Taking CouchSurfing as the context, this research answers several questions: 1 ) what is the motivation of Indonesian CouchSurfing members to host? 2) Through what practices is value co-created between host and guest in CouchSurfing? 3) What resources are needed to co-create value in the context? 4) What value emerges from hosting in CouchSurfing?

Semi-structured interviews were conducted with 20 participants from Bandung, Yogyakarta, and Bali. In addition to the individual interviews, a focus group discussion with 6 participants was held in Yogyakarta. Findings from this research highlight the intrinsic, personal and emotion-based nature of hosts' motivations to participate in Couchsurfing, including the establishment of personal connections and friendships, an opportunity to behave in altruistic ways, and the increase of knowledge through other people's lives and experiences. To fulfil these motivations to the best possible extent, guest 
selection strategies were put into place by hosts, based on prior experience. The co-creational social practices then closely aligned to those motivations and are regulated by hosts' management strategies. This ensured a positive experience, resulting in a variety of value outcomes including new knowledge, positive selfidentity as well as professional opportunities.

Findings of this research also highlight the unique nature of co-creational experiences in non-monetary accommodation sharing and the unusually strong impact of motivations on all components of the value co-creation process. This research concludes with suggestions for future research and both theoretical and practical implications for harnessing intimacy and authenticity in the tourism industry. 


\section{Acknowledgement}

These past two years has been a challenging and amazing journey, but also years full of encouragement, support and inspiration from innumerable people. These past two years showed that I am lucky for being surrounded by many people who keep my level of enthusiasm high and put the words 'give up' away from my life.

Most importantly, I would like to thank to New Zealand Aid Scholarship for giving me support and opportunity to make my dream come true. New Zealand, especially Wellington, has treated me well and I have had an amazing experience here. I learned from the experts and hopefully I can contribute what I have learned here back home.

I owe myself gratitude for always searching motivation to keep going and never surrender, even in the worst possible state of mind. For Marsello, my dear son, being a happy single parent to you was the only idea came to my mind that saved me from ending this masters journey in the first three months. Without you, without the idea, I think I will never be in this point, regaining my happiness and confidence to face the future. For that, I am very grateful to you. Also for my parents and my sisters, I thank you all for the love. There will be no thesis or maybe even a masters degree without your encouragements and support.

Huge thanks to Ina Reichenberger, your advice and support are invaluable. I am so lucky to have the opportunity to learn a lot from you, but at the same time you let me enjoy the process of learning itself. It is so lucky to have a supervisor I could laugh with during the meeting but who would still keep encouraging me to meet the objectives. Thank you for trusting me.

I would also thank to all professors of SOM, especially Eerang Park, for giving me a chance to do master thesis; Mondher Sahli, Ian yeoman, and Douglas Pearce for the support during my master journey. It was an honor to be able to learn from you. For Megan Key and Louisa Acheson, I thank you for the incredible 
administrative support. To my honor and master colleagues, it has been a pleasure to share this experience with you.

To VUW International Student Officer (ISO), especially Ryan Stuart, thank you for your support since the very beginning I arrived in New Zealand and started this journey, for lending me an ear and encouraging me not to quit the journey. Thank you for convincing me that New Zealand Aid Scholarship never made a mistake in selecting the awardees. I hope I proved that.

To all my participants, I thank you for spending the time with me and sharing your experience. You have provided invaluable contribution to my thesis. Finally, to my Indonesian fellow students, I am glad to have incredible and supportive friends when away from family. For that, I know that I am so lucky! 


\section{Table of Contents}

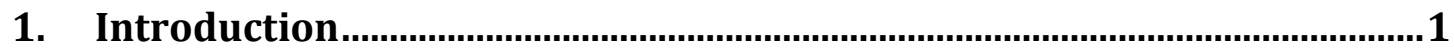

1.1. General Introduction ...................................................................................... 1

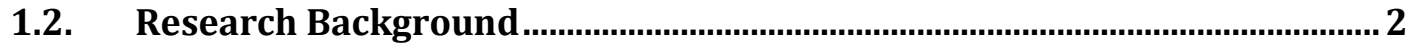

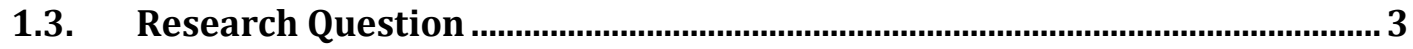

1.4. The Research Context: Tourism in Indonesia and CouchSurfing................... 5

1.4.1. General Background of Tourism in Indonesia .....................................................6

1.4.2. Couchsurfing as the Research Context.........................................................................

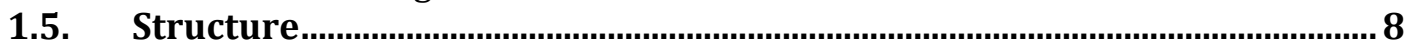

2. Literature Review .............................................................................. 10

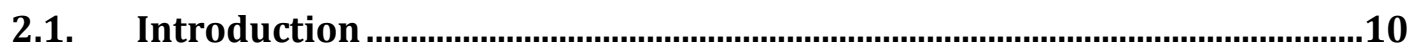

2.2. Motivation..............................................................................................11

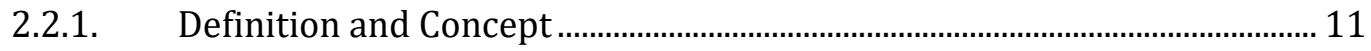

2.2.2. Social Exchange Theory ……………………………………………………….... 12

2.2.2.1. Resources of Exchange ........................................................................................................ 13

2.2.2.2. Rules of Exchange ..............................................................................................13

2.2.2.3. Review of Social Exchange Theory ..................................................................... 14

2.2.3. Social Capital Theory ………………………………………………………….... 15

2.2.3.1. Structural Factors ........................................................................................................... 17

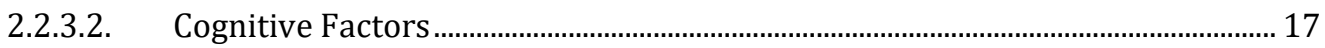

2.2.3.3. Relational Factors ............................................................................................................. 18

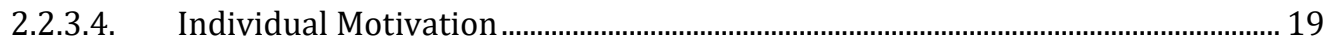

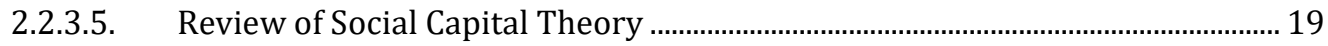

2.2.4. Motivation in Collaborative Consumption..................................................... 20

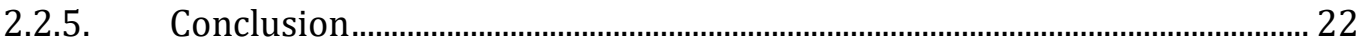

2.3. Value Co-creation ......................................................................................22

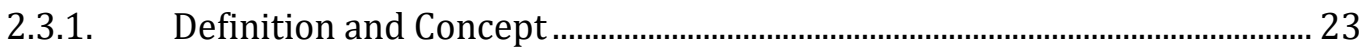

2.3.2. Value Co-Creation in Hospitality Context.............................................................. 24

2.3.2.1. Value Co-creation from the Tourist's Perspective.................................................. 25

2.3.2.2. Value Co-creation from Tourism Service Provider's Perspective ........................26

2.3.2.3. Review on Value Co-creation in a Hospitality Context..........................................29

2.3.3. Value Co-Creation in Collaborative Consumption........................................... 30

2.3.4. Conceptual Framework ........................................................................................... 32

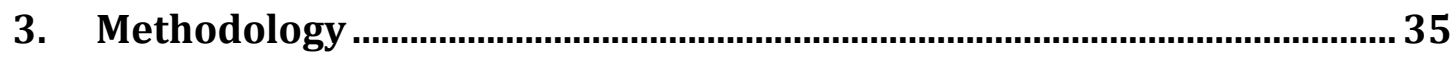

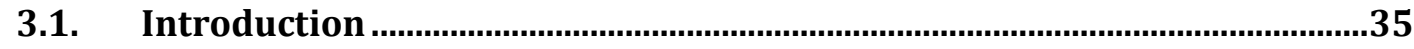

3.2. $\quad$ Research Questions .......................................................................................35

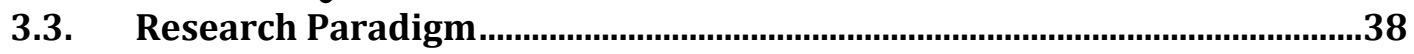

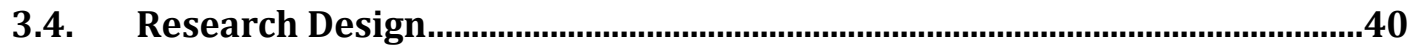

3.4.1. Qualitative Research............................................................................................ 40

3.4.2. Semi-Structured Interviews .................................................................................... 41

3.4.2.1. Interview Guide........................................................................................................................... 42

3.4.2.2. Sampling ......................................................................................................................................... 43

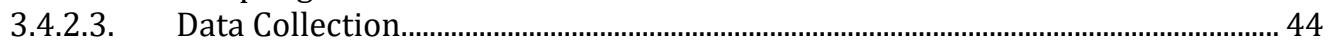

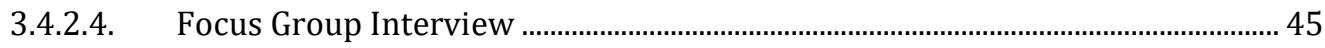

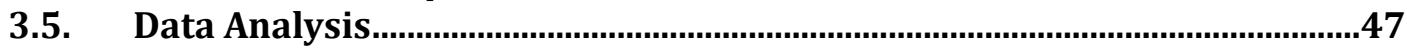

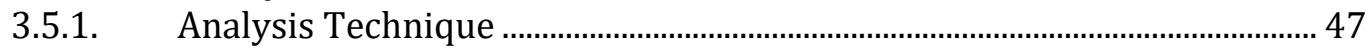

3.5.2. Analytical Framework........................................................................................ 48

3.6. Researcher Position in the Research ……………....................................51

3.7. Research Limitation and Strength ……………………………………....51

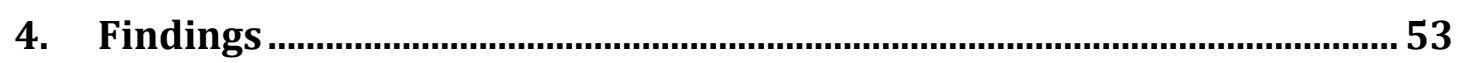

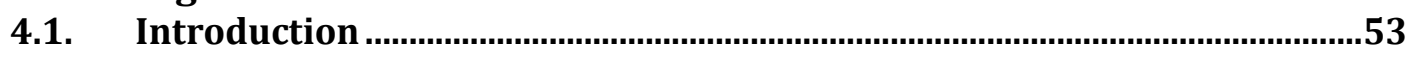

4.2. Sample Characteristics................................................................................53 
4.3. The Motivation for Non-Monetary Accommodation Sharing …...................55

4.3.1. Emotional Needs ........................................................................................... 57

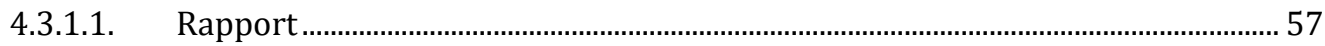

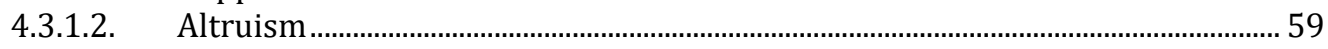

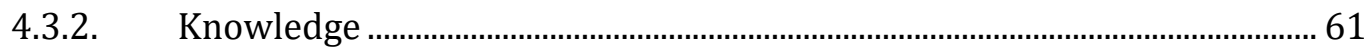

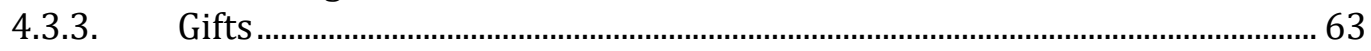

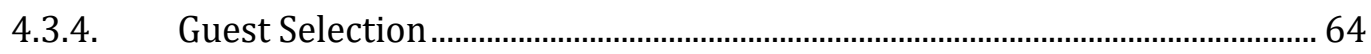

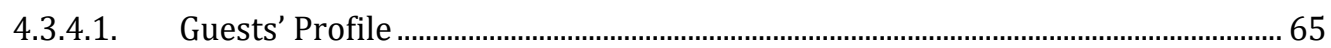

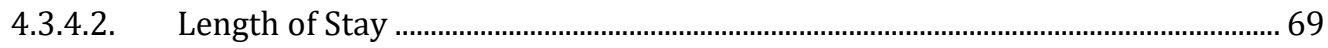

4.3.5. Conclusion Summary.................................................................................... 70

4.4. Value Co-Creation Practice …....................................................................

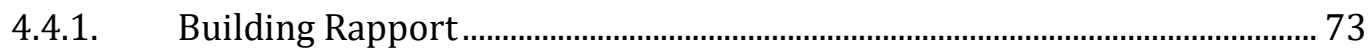

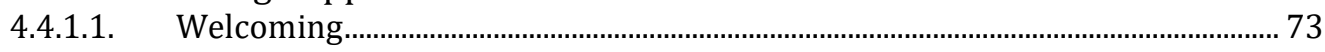

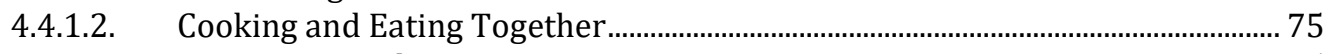

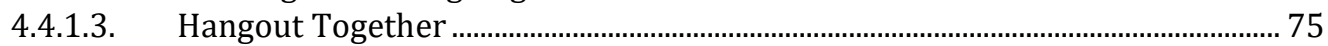

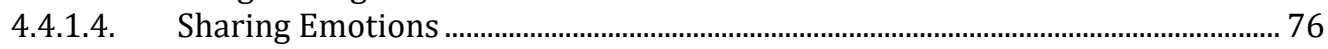

4.4.1.5. Touring with Guest.................................................................................................... 77

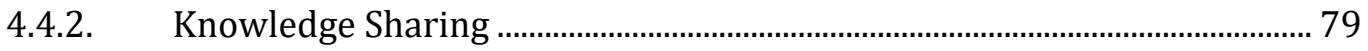

4.4.2.1. Deep Conversation............................................................................................. 79

4.4.2.2. Providing Tips and Advice .................................................................................... 80

4.4.2.3. Learning New Skills........................................................................................... 81

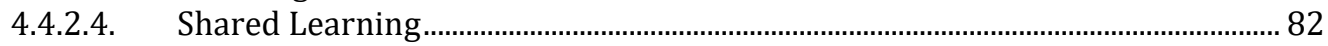

4.4.3. Insight into Local Life .................................................................................... 82

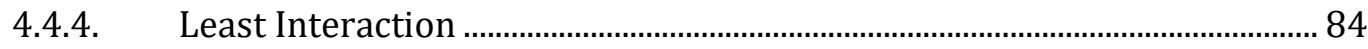

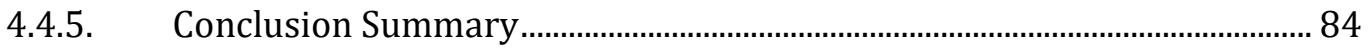

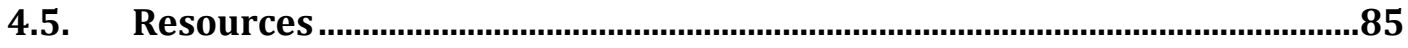

4.5.1. Management Strategies .................................................................................... 86

4.5.2. Host's Knowledge.............................................................................................. 88

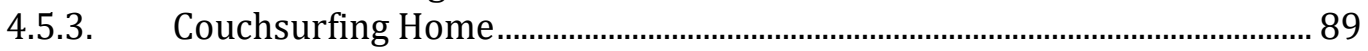

4.5.4. Conclusion Summary ................................................................................. 91

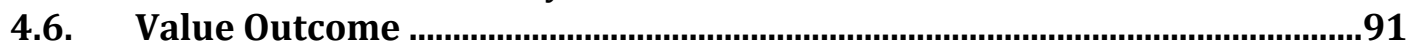

4.6.1. Rapport.......................................................................................................... 93

4.6.2. Knowledge .................................................................................................... 94

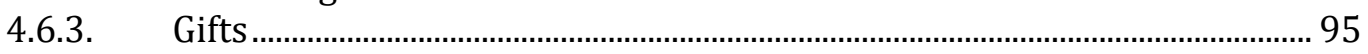

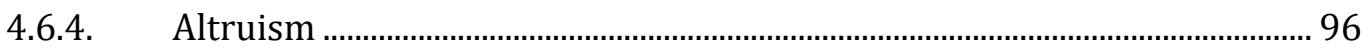

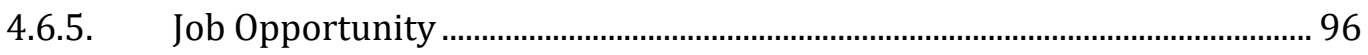

4.6.6. Conclusion Summary ...................................................................................... 97

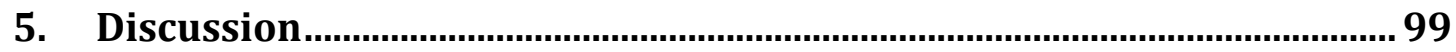

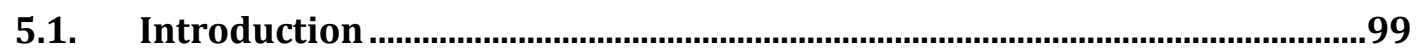

5.2. The Motivation for Non-Monetary Accommodation Sharing .....................99

5.3. Value Co-Creation Practice .....................................................................104

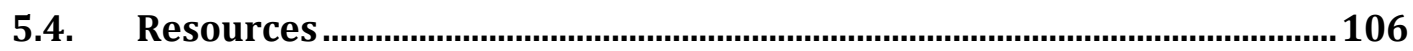

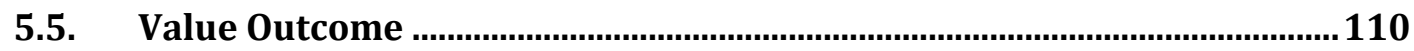

5.6. A Revised Conceptual Framework ..........................................................113

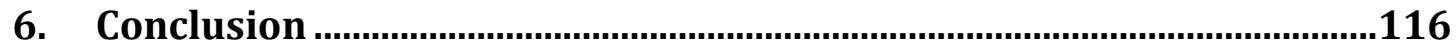

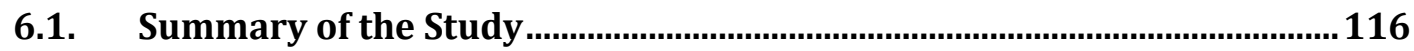

6.2. Limitation and Future Research .......................................................... 118

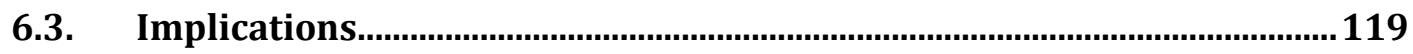

6.3.1. Theoretical implication .........................................................................120

6.3.2. Practical implications ................................................................................ 120

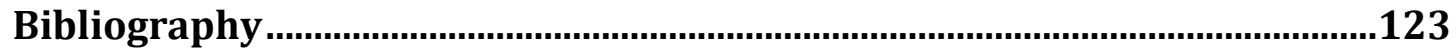

Appendices............................................................................................................129

Appendix A: Information sheet for participants ................................................. 130 
Appendix B: Information sheet for Focus Group Discussion .................................. 132

Appendix C: Consent Form .............................................................................. 134

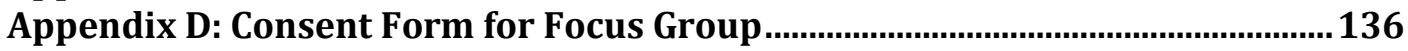

Appendix E: Interview Guidelines .................................................................... 138

Appendix F: Interview Guidelines for Focus Group ............................................... 140 


\section{List of Tables}

Table 3.1: Interview Guidelines Mapping............................................................... 43

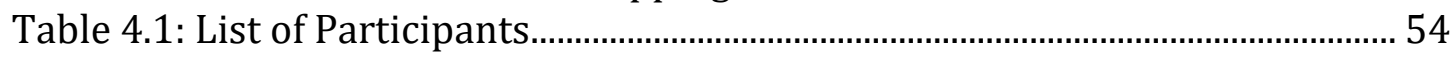

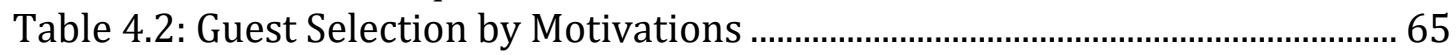

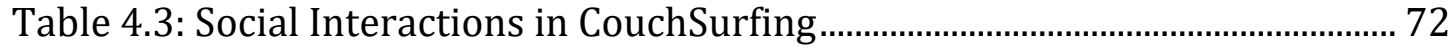

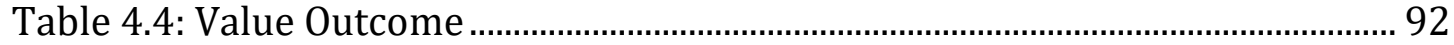




\section{List of Figures}

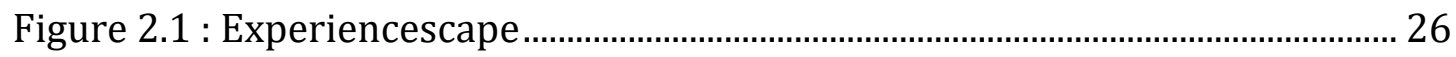

Figure 2.2 : Conceptual Framework of Hospitality Intelligence................................ 28

Figure 2.3 : Conceptual Framework for Value Co-creation in CouchSurfing......... 34

Figure 3.1 : Analytical Framework for Value Co-creation in CouchSurfing ........... 50

Figure 4.1 : Motivation in CouchSurfing ……............................................................ 56

Figure 4.2 : Resource for Value Co-creation............................................................... 86

Figure 5.1: Revised conceptual framework of Value Co-creation

in CouchSurfing 


\section{Introduction}

\subsection{General Introduction}

Collaborative consumption allows individuals to share access and offers value with less cost (Botsman \& Rogers, 2010; Hamari, Sjöklint, \& Ukkonen, 2016). In the context of accommodation sharing, collaborative consumption platform is a low-cost alternative to more traditional approaches to accommodation and one which can provide tourists with the opportunity to have direct interaction with hosts and local communities (Lampinen \& Cheshire, 2016; Tussyadiah \& Pesonen, 2016). Thus, collaborative consumption fosters memorable tourism experiences while at the same time providing the tourism industry with a diversification of accommodation choice.

Airbnb and CouchSurfing exemplify two of the most popular avenues for accommodation sharing. Airbnb is representative of monetary platforms and has gained much attention in the academic literature concerning tourism experiences (Johnson \& Neuhofer, 2017; Lampinen \& Cheshire, 2016; Tussyadiah \& Pesonen, 2016). Unlike Airbnb, CouchSurfing is an accommodation sharing without financial involvement as the exchange. Current research postulates that by the absenting of financial involvement, CouchSurfing offers more intimacy and experience in the host/guest relationship than monetary accommodation sharing (Decrop, Del Chiappa, Mallargé, \& Zidda, 2017; Molz, 2012; Schuckert, Peters, \& Pilz, 2017). However, little is known about the perspective of hosts who provide non-monetary accommodation sharing and the characteristics of host/guest relationship in the platform.

Meanwhile, there has also been a shift in tourist behaviour, in which tourists are now seen as the creator of their own experience (Cabiddu, Lui, \& Piccoli, 2013; Johnson \& Neuhofer, 2017; Prahalad \& Ramaswamy, 2004; Shaw, Bailey, \& Williams, 2011). They are no longer seen as "passive sightseers consuming sites in prescribed fashions" (Ek, Larsen, Hornskov, \& Mansfeldt, 2008, p. 126) but as those who want to participate actively and do multi-sensory exploration in the destination with other subjects (Campos, Mendes, do Valle, \& Scott, 2016; Campos, Mendes, Valle, \& Scott, 2015; Prahalad \& Ramaswamy, 
2004). Consequently, value is no longer determined and created by tourism service providers but through "joint creation of value" (Prahalad \& Ramaswamy, 2004, p. 8) with tourists.

Little is known regarding how social interaction occurs between host and guest in the context of interrelated value co-creation and non-monetary accommodation sharing. The absence of financial involvement means that the value and how the host derives value remain vague. Therefore, motivated by the notion of value co-creation and non-monetary accommodation sharing, this study aims at contributing to the conceptualisation of value co-creation in CouchSurfing from the perspective of the host, and examines factors that may contribute to the hosting experience.

As an introduction to the research, this chapter will first outline the importance of the study to the academic literature and describe the research context. Subsequently, the research questions as the fundamental core and guiding for the research will be introduced followed by the structure of the thesis.

\subsection{Research Background}

Shifts in tourist behaviour have impact on value creation in the tourism industry (Ek et al., 2008; Tussyadiah \& Pesonen, 2016). Tourists nowadays no longer perceive tourism activities as a way of seeing (Ek et al., 2008). Instead, they have changed into those who participate and engage in activities in the destination with other subjects to create a memorable experience (Campos et al., 2016; Campos et al., 2015; Prahalad \& Ramaswamy, 2004). The means that the tourism industry can no longer function as a service provider, in which they create value by selling service to be monetarily exchanged to tourists. Because of changing tourist behaviour, tourism providers are required to become experience providers and along with tourists, they co-create value through experience.

Campos et al. (2016) describe co-creation in the tourism industry as "a process of interrelated interactions and activities that connects the tourist and other actors, and experiences are the context in which those interactions and 
activities occur" (p. 1311). Apart from the fact that human interaction has already been acknowledged to provide significant substance in the tourism and hospitality industries (Campos et al., 2015), the study of co-creation perceives interaction between actors as a source of experience, in which they influence each other's lives to co-create value (Bharwani \& Jauhari, 2013; Campos et al., 2016; Campos et al., 2015; Prahalad \& Ramaswamy, 2004; Shaw et al., 2011; S. L. Vargo, Maglio, \& Akaka, 2008).

While the existing literature on value co-creation in the tourism industry has been significant, most of the research so far focuses on monetary accommodation providers. With financial involvement characterising the relationship between tourist and accommodation provider, the value derived from the experience is a business oriented. Thus, there is a gap in research in how the characteristics of the relationship between actors in non-monetary accommodation sharing take place, and little is known about how value cocreation proses occur in this context. In addition, there is a gap in the academic literature concerning the motivation encouraging individuals to join the platform.

In order to provide answers to these gaps, this research aims at contributing to the conceptualisation of value co-creation in non-monetary accommodation sharing by exploring the interactions occur between host and guest during the stay, the resources needed, and value as an outcome. However, before examining the process of value co-creation, the motivation of individuals to join the platform will first be explored. By so doing, this research provides a comprehensive investigation into the process of value co-creation, starting from the encouraging factors to host and how these factors exert influence on the process of value co-creation.

\subsection{Research Question}

As the extant literature on value co-creation in the hospitality context focuses more on monetary accommodation than non-monetary accommodation sharing, a comprehensive investigation to the value co-creation practice in CouchSurfing context is required. Particularly, motivation to join the platform 
and enter social interaction has not been addressed, and the process of value cocreation in the context is unclear. Drawing upon the conceptual framework of value co-creation, this study aims at addressing the following research questions:

1) What is the motivation of Indonesian CouchSurfing members to host?

Research question 1 explores the motivation and contributing factors in the decision to become a host with CouchSurfing. More specifically, this research question focuses on the rewards expected by Indonesian CouchSurfing members when they host. Knowing the motivation of hosts provides insight into the notion of taking and giving through the accommodation platform and how it impacts on the way hosts conduct social interactions and co-create value.

\section{2) Through what practices are values co-created between host and guest in CouchSurfing context?}

Research question 2 investigates which and how social interaction occurs between host and guests during CouchSurfing encounters. This research question aims to understand how the on-site co-creation experience characterises the host/guest relationship in CouchSurfing. Therefore, this research question will explore what types of interactions occur between host and guest and the purpose to which they conduct the interactions. Understanding this perspective will provide a foundation for understanding how values are co-created, including resources needed in the interactions.

\section{3) Which resources are needed for these social practices?}

The literature on value co-creation postulates that values are cocreated through the integration of resource and value co-creation practice (S. L. Vargo et al., 2008). This premise implies that to co-create value, actors will harness the resources and apply them to social interactions. Therefore, this research question will explore what resources exert their 
effect on value co-creation practices, and the way the hosts harness the resources of the interactions.

\section{4) What values emerge as outcomes for hosting in CouchSurfing?}

As motivation and value co-creation practices and resources as an integrated value co-creation process has been elaborated, value, as an outcome for the process, will now be addressed. This research question will examine how values are co-created from social interaction by drawing together findings from research question 1 to 3 . Further, this research question will interrelate value to motivation in order to examine how these parts are interrelated.

Providing answers to these questions, the study's main contribution lies in its theoretical framework grounded in motivation and co-creation by taking the context of non-monetary accommodation sharing into account. Further, this study also examines to what extent the concept of value co-creation is applicable in non-commercial accommodation sharing contexts. In addition to its academic contribution, this study provides insight for destination managers to set strategy in developing competitive tourism destinations. Through value co-created by the interaction between host and guest, destination managers could develop marketing platforms that offer intimacy and local authenticity in a destination. Finally, through the practice of social interaction, tourism destinations could benefit from skill development, language and cultural understanding.

\subsection{The Research Context: Tourism in Indonesia and CouchSurfing} Indonesia and CouchSurfing as the research context will strongly influence the methodological approach to this research. They will determine the characters of the sample that may be distinguished from members of another accommodation sharing platform and culture. Consequently, the character of the samples will reflect their motivation to join the platform and the relationship with guests during the encounters. 


\subsubsection{General Background of Tourism in Indonesia}

Using the slogan 'United in Diversity' (Bhineka Tunggal Ika), Indonesia declares itself as a country with at least 1300 ethnic groups (BPS, 2018) . Scattered across more than 17,500 islands and 34 provinces (BPS, 2018), each ethnic groups possesses its own set of customs and unique cultural values and objects (Leinbach, Mohamad, Wolters, \& Adam, 2019). Therefore, it is challenging to define and describe the general culture of Indonesia because there is no such Indonesian culture as one.

However, when it comes to tourism, Indonesia is the country with the highest tourism growth in South East Asia (WTTC, 2018). The country's natural and cultural assets have become major tourist attractions. With 15,806,191 international visitor arrivals (WTTC, 2018) and a contribution to the national GDP of USD 58,9 billion in 2017 (WTTC, 2018), tourism has become one of the leading sectors of the economy in Indonesia. Bali is one of the provinces with the most significant contribution to the tourism industry (BPS, 2018). With its natural and distinctive cultural assets, Bali has become the main point of entry for international tourists arriving in Indonesia (BPS, 2018).

\subsubsection{Couchsurfing as the Research Context}

CouchSurfing as the research context will strongly influence the methodological approach to this research. It determines the characteristics of the sample, who may be distinguished from members of another accommodation sharing platform. Consequently, the character of the samples will reflect their motivation to join the platform and the relationship with guests during the encounters.

Couchsurfing is one of the famous non-commercial accommodation sharing platforms, with the mission to create a better world through fostering cultural exchange and mutual respect (Couchsurfing, 2017). Therefore, CouchSurfing is well-known as "a web-based network for travellers who like to meet and interact with locals at their destination" (Schuckert et al., 2017, p. 2). It provides access to local life and authenticity while at the same time travellers can stay for free in a 
local house, although some hosts are only available for coffee or showing the travellers around town (Molz, 2012).

Putting aside financial motivation, Couchsurfing has a social mission in enabling people to meet new acquaintances and make connections through an online platform (Chen, 2012; Decrop et al., 2017; Molz, 2012). Through the site, members are enabled to search for a host in a particular destination, make 'couch' requests to stay with hosts, participate in active member discussion boards, or join groups based on shared interests (Molz, 2012). In order to see if host and guest will make compatible housemates, both of them can consult each other's profile pages for more information (Molz, 2012) or communicate to each other through e-mails and maybe phone calls.

Couchsurfing's interface is similar to other online social networking sites like Facebook, in which each member has an online profile with the details of their personal information, such as a photograph of themselves, age, gender, hometown, previous travelling experience, interests, and philosophy on life. However, unlike Facebook, most of the members' motivations to join the site is not to keep in contact with pre-existing friends but to connect to strangers (Rosen, Lafontaine, \& Hendrickson, 2011). Thus, it can be assumed that CouchSurfing members are those who want to experience personal and emotional connections and get involved in various social interactions with locals, which afford them with intimacy and familiarity with their destinations (Schuckert et al., 2017). However, Couchsurfing blurs the distinction between host and guest by encouraging guests also to be willing to offer their accommodation to travellers in need (Molz, 2012). With the role of the members shifting fluidly between host and guest, "the performances between 'hosting' and 'guesting' are relational" (Molz, 2012, p. 218). Hosts may be potential guests who happen to be at home, while guests are potential hosts who happen to be on the road (Molz, 2012).

CouchSurfing can be seen as "hospitality experience" (Schuckert et al., 2017, p. 2) characterised, and co-created by the host/guest relationship (P. Chathoth, Altinay, Harrington, Okumus, \& Chan, 2013; Schuckert et al., 2017). Further, Schuckert et al. (2017) argue that in addition to the ordinary hospitality 
products, CouchSurfing represents a cultural exchange and accommodation experience (Schuckert et al., 2017).

\subsection{Structure}

This thesis is structured into six chapters. The first chapter is the introduction, which outlines the research gaps in the academic literature on value co-creation in tourism. Following from these gaps, this chapter provided the foundation for the research presented in this thesis and formulated it through the research questions. Further, it introduced CouchSurfing as the research context and outlined the aim of the network and the general character of the members.

Chapter 2 is a literature review, and presents the relevant information related to motivation and value co-creation from the current literature. Firstly, the discourse on motivation in the academic literature will be presented to provide insight into the contributing factors of individuals' decision to host on CouchSurfing. Secondly, the literature on value co-creation will be presented to illuminate the process of value creation, starting from the definition and context of value co-creation in general, in the hospitality context, and value co-creation in collaborative consumption. At the end of the chapter, a conceptual framework will be presented as an integration of the literature and foundation for the methodology.

Chapter 3 will describe the methodology of the research, which provides information on the design of the study. First, the research questions will be revisited, and the data needed for each question will be outlined. The research paradigm then will explain the approach of the study, which will determine the technique of data collection and data analysis. At the end of the chapter, an analytical framework will be presented as a guide for data analysis and the structure of the findings. Chapter 4 will then explain the findings of the research, starting from the profile of the sampling, which provides the characteristics of the respondents of the research. This chapter is divided into four parts based on the sequence of value co-creation process, starting from motivation, value cocreation practice, resource and value. 
Chapter 5 is the discussion, which describes the academic contribution of the findings related to existing literature. This chapter is also divided into four parts based on the sequential process of value co-creation. As a summary of the academic contribution of this research, a revised conceptual framework will be presented at the end of the chapter. Finally, the last chapter is the conclusion to the study, and provides a summary of the study. This chapter will outline the theoretical and practical contribution to the research. The limitations of the research and the implications for future research will also be provided. 


\section{Literature Review}

\subsection{Introduction}

As explained in the introduction, while extant research on value cocreation has been undertaken in various tourism contexts from different perspectives, there are gaps in the literature on how the notion of value cocreation occurs in non-monetary accommodation sharing. Seen from the host perspective, this research aims to analyse the process of value co-creation by including motivation in the process to provide a comprehensive understanding of the hosting experience.

In order to provide a critical analysis of the current academic literature on motivation and value co-creation, this chapter will provide a review of the discourse of motivation and value co-creation. In the beginning, the literature on motivation be presented. Discussion on this section includes the definition of motivation in the tourism study, followed by motivation in the tourism context. Subsequently, social exchange and social capital theory will be elaborated to provide relevant information to examine hosting decisions and contributing factors that may relate to these decisions. Then, motivation on collaborative consumption will be delineated to put the theory into the research context.

The second part of the literature review will discuss the notion of value co-creation. In this part, firstly the definition and concept of value co-creation, in general, will be presented to provide an overview of the paradigm. Following the definition and concept, a section on value co-creation in the hospitality context presents a range of information related to current academic literature. Similarly to the reviewed literature on motivation, information on value co-creation in the context of collaborative consumption will be presented, as a foundation to discuss what is known in the research context related to current paradigms. To conclude, a conceptual framework will be introduced, which serves as the basis for the methodology employed in this research. 


\subsection{Motivation}

This chapter aims at providing a theoretical background on the motivation of individuals to host in CouchSurfing. Therefore, this chapter will first discuss the definition of motivation in general and in the tourism context. Secondly, social exchange and social capital theory will be introduced to explain the contributing factors related to hosting decisions. Lastly, motivation in collaborative consumption will be presented to scrutinise the motivation of individuals to join collaborative consumption platforms.

\subsubsection{Definition and Concept}

In order to provide a basic understanding of motivation, this section will discuss the definition and concept of motivation in general and in the tourism context. The discussion will include the definition and the impacts of motivation on individuals' behaviour.

The study of motivation is rooted in psychology. In tourism studies, the studies on motivation are used to understand the drivers encouraging tourists to come to a particular destination, and their decision-making and overall evaluation toward their holiday's consumption (Goeldner, 2009). In short, understanding motivation in the tourism context is a starting point for studying tourist behaviour and further understanding of the tourism system and development (Getz, 1986).

Motivation is seen as the influential force behind all behaviours (Fodness, 1994). In the study of tourism, P. L. Pearce (1998) defined tourist motivation as "the global integrating network of biological and cultural forces which gives values and direction to travel choices, behaviour and experience" (p. 33). Therefore, Gianna Moscardo, Morrison, Pearce, Lang, and O'Leary (1996); P. L. Pearce (1998) develop the travel career ladder framework, to illustrate a range of individual motives for pursuing holiday experiences, based on the theory of motivation proposed by Maslow. Maslow's framework organised the needs of travellers into a ladder with the relaxation needs at the lowest level, followed consecutively by safety needs, relationships needs, self-esteem, development needs and fulfilment needs at the highest level (P. L. Pearce \& Lee, 2005). 
However, in the latest approach, P. L. Pearce and Lee (2005) modified the framework into Travel Career Pattern (TCP), emphasising the pattern of motivation rather than a hierarchical ladder.

Understanding tourist motivation gives insight into the demands that influence destination choice, particularly travellers' satisfaction of their needs. To be exact, travellers will go to a certain destination where they can do certain activities that suit their personal psychological and motivational profiles (Goeldner, 2009). For this study, discourse on motivation will be taken from the supply perspective. Adapting the tourist' motivation perspective, it can be concluded that any individuals' actions are in the pursuit of satisfying their needs.

\subsubsection{Social Exchange Theory}

As the definition and general premise of motivation have been understood, the social exchange theory will now be presented as a theoretical foundation to examine motivation in CouchSurfing.

Ap (1992) refers to social exchange theory as "a general sociological theory to understand the exchange resources between individuals and groups in an interaction situation" (p. 668). Therefore, understanding individual's motivation to host in CouchSurfing using social exchange theory is based on the premise that human behaviour is an exchange of activity, tangible and intangible (G. C Homans, 1961; George Caspar Homans, 1961). Argyle (1981) further suggests that individuals decide to engage in interaction as they expect specific rewards. This decision has been previously structured through the analysis of benefits and costs, which later influence the pattern of interaction (Nunkoo, 2016). Therefore, interactions may continue if both parties perceive that they gain more benefit from the exchange (Ap, 1992; Nunkoo, 2016; P. L. Pearce \& Lee, 2005; Ward \& Berno, 2011).

In examining social behaviour, social exchange theory is considered as one of the oldest theories (Nunkoo, 2016), which strongly emphasises social interaction and relationships as an exchange process (Blau, 1964). These interactions and relationships are regarded as a process whereby actors provide 
one another with valued resources (Ap, 1992). However, social exchange is different from economic exchange in several fundamental ways. Instead of being formal and often contractual, benefits in social exchange are rarely negotiated (Blau, 1964). Exchange of benefits is also a voluntary action and involves unspecified future responsibilities (Ap, 1992). Therefore, there is no guarantee that there will be a reciprocal benefits although there is an expectation of future returns for the contributions between exchange actors, regardless the unknown or negotiated nature of the returns (Blau, 1964).

\subsubsection{Resources of Exchange}

Apart from the nature of the returns, individuals are driven by needs and satisfaction in deciding to engage in exchange relationships (Ap, 1992; Waitt, 2003). In term of resources of exchange, Blau (1964) distinguishes between intrinsic and extrinsic rewards. Intrinsic rewards can manifest in the form of love and respect, while extrinsic rewards can be money or job opportunities. However, building upon social exchange theory, B. F Meeker (1971) later identifies resources of exchange into six items, i.e., love, status, information, money, goods and services.

\subsubsection{Rules of Exchange}

Interpersonal exchanges can be considered individual, personal decisions (B. F. Meeker, 1971). Therefore, individuals require rules to guide the decisions they have made (Cropanzano \& Mitchell, 2016). Based upon this B. F. Meeker (1971) posits six underlying rules: reciprocity, rationality, altruism, group gain, status consistency, and competition.

Reciprocity is characterised as interdependence, meaning that it involves a mutual and complementary arrangement between parties (L. D. Molm, 1994). Accordingly, the outcomes of the exchange are based on the effort and collaboration between parties. Further, a reciprocal exchange is understood as one that does not involve explicit bargaining (L. Molm, 2003). Instead, an action of one actor depends on another's behaviour, meaning that an action of one party leads to a reaction by another. Therefore, the process of reciprocal 
exchange commences when at least one participant initiates an action and if the other reciprocates, new cycles of exchange begin (Cropanzano \& Mitchell, 2005, 2016).

Rationality refers to using logic to determine the expected outcomes and how one should achieve those valued outcomes (Cropanzano \& Mitchell, 2005, 2016; Hellwing, Morhart, Kocher, \& Zisiadis; B. F. Meeker, 1971). A rational action has two parts - "an end of value maximisation and a means of logic" (Cropanzano \& Mitchell, 2016, p. 879). However, B. F. Meeker (1971) also argues that one does not always act rationally. Therefore, other principles of social exchange are added.

Altruism is a rule in which people try to benefit another person even at cost to themselves. Conceptualising altruism refers to broad personal motives that drive people to help others (Wilson, 2015). Group gain does not include interpersonal exchange because all things are held in common. According to the group gain rule, the benefits are put into "a single common pot" (Cropanzano \& Mitchell, 2005, p. 879), where individuals take what they need from this shared pool regardless of their particular contribution. Status consistency or rank equilibration is the distribution of benefits based on one's position within a social group (Cropanzano \& Mitchell, 2016). Legacy status, then, is "a status consideration" (Cropanzano \& Mitchell, 2016, p. 879) where someone with a higher rank will get more significant benefits. Meanwhile, competition is the opposite of altruism (Cropanzano \& Mitchell, 2016). Where altruism is assisting others even when it potentially upsets oneself, competition is maximising one's earnings even when it harms others (B. F Meeker, 1971).

\subsubsection{Review of Social Exchange Theory}

Social exchange theory has been popular in anthropology, sociology and social psychology studies concerned with the perception of the relative costs and benefits of relationships and their consequences for relationship satisfaction (Ap, 1992; Cropanzano \& Mitchell, 2005; Waitt, 2003; Ward \& Berno, 2011). In the tourism context, social exchange theory is applied to examine the perception of host residents toward tourism development (Andriotis \& Vaughan, 2016; Hsieh, 
Tsai, \& Chen, 2017; Nunkoo, 2016; P. L. Pearce \& Lee, 2005; Ward \& Berno, 2011). According to the literature, residents tend to support tourism developments, as they perceive them to have positive economic, environmental, and sociocultural impacts (Nunkoo, 2016; Paraskevaidis \& Andriotis, 2017; Ward \& Berno, 2011). In that context, social exchange theory has provided a conceptual foundation for the examination of inter-relationships among perceptions of costs and rewards, positive and negative impacts, and support for tourism. Being applied at the individual level, social exchange theory proposes an explanation for the robust findings that those employed in the tourism sector, who receive more direct benefits from the industry, have more positive attitudes toward tourists and tourism development (Haley, Snaith, \& Miller, 2005).

Regardless, it is still unknown which rules apply to which resources (Cropanzano \& Mitchell, 2005, 2016). Different resources may be exchanged in different ways. However, although the theory has been widely used in tourism studies to explain the perception of residents toward tourism development, most studies only focus on extrinsic goals (Paraskevaidis \& Andriotis, 2017). They concern economic benefits such as personal income and employment, but lack research on intrinsic and intangible rewards. Meanwhile, this research takes the context of CouchSurfing, in which there is no financial involvement in the relationship of actors within. In order to fill this gap, social capital theory will be used in conjunction with social exchange theory. This theory provides an explanation of the motivation to host on CouchSurfing through the relationship of its members with CouchSurfing as a network.

\subsubsection{Social Capital Theory}

The previous section presented an overview of social exchange theory and its application in the tourism industry. The theory suggests that individuals seek involvement in social interaction as they expect specific rewards. However, a review of social exchange theory concerning its application in tourism studies implies that it is not adequate to fully explain motivation in the context of tourism. Therefore, the social capital theory will now be presented as a theoretical companion to social exchange theory by which to examine 
CouchSurfing as a network.

The idea of social capital comes from an additional feature to elements that build organisational capital: physical capital, financial capital, and human capital (Huysman \& Wulf, 2005). Unlike human capital, which refers to individual capability (Becker, 1975), social capital signifies the collective abilities gained from social networks (Huysman \& Wulf, 2004). Thus, in the organisational context, social capital can be described as "features of social organisation such as networks, norms, and social trust that facilitate coordination and cooperation for mutual benefit" (Putnam, 1995, p. 2).

Nahapiet and Ghoshal (1998) identified social capital as "the sum of the actual and potential resources embedded within, available through, and derived from the network of relationships possessed by an individual or social unit" (p. 243). Later on, G. Moscardo (2014) defined social capital as "the stock of goodwill, potential assistance, and access to resources that individuals and groups may have" (p. 69). In other words, networks of relationships form a valuable resource, which provides the members of the network with collectively intangible capital (Nahapiet \& Ghoshal, 1998).

Social capital inhabits the structure of relationships between individuals and in individuals' relationship with their networks (Wasko, McLure, \& Faraj, 2005). Social capital can only appear from social networks characterised by common obligations, shared norms and values, and relationships built on trust and reciprocity (Flora, 2004; Gianna Moscardo, Konovalov, Murphy, McGehee, \& Schurmann, 2017). In these networks, interaction and connections between members develop into shared trust, norms, and reciprocity that promote collaboration to accomplish common goals (Ecclestone \& Field, 2003). Having social capital, as long as individuals are within the network, they can expect rewards (tangible/ intangible) from their social networks, even if the rewards might not generate immediate economic benefits (Kim, Lee, Koo, \& Yang, 2017).

Nahapiet and Ghoshal (1998) suggest social capital theory as an integrative framework for understanding the sharing and creation of knowledge in organisations. To justify intellectual capital creation within organisations on a group level, Nahapiet and Ghoshal (1998) suggested that knowledge sharing and creation within the network can be facilitated under these conditions: 1) 
individuals are encouraged to engage in its exchange, 2) there are connections between individuals (structural capital), 3) individuals possess the cognitive capability to understand and apply the knowledge (cognitive capital), and 4) the relationship between individuals is stable and possesses positive characteristics (relational capital).

\subsubsection{Structural Factors}

It is assumed that the connections between individuals in a network determine the collective action of the members. When a network consists of a huge proportion of strong, direct connections between members, collective action is comparatively easy to achieve (Nahapiet \& Ghoshal, 1998), and it is more likely that members will develop a habit of cooperation and collaboration (Marwell, Oliver, \& Prahl, 1988). In examining individual actions, Wasko et al. (2005) suggest that individuals who are centrally embedded and are likely to have developed habit of cooperation tend to understand and comply with group norms and expectations .

\subsubsection{Cognitive Factors}

Cognitive factor refers to "those resources that make possible shared interpretations and meanings within a collective" (Wasko et al., 2005, p. 41). This capital involves of both individual expertise and mastering the application of expertise, which takes experience (Nahapiet \& Ghoshal, 1998; M. M. Wasko \& Faraj, 2000; Molly McLure Wasko \& Samer Faraj, 2005). Individuals' expertise and abilities should increase their possibility to contribute to the network. Therefore, those who are motivated to contribute within the network are individuals who possess the resources. On the contrary, individuals who think that they have inadequate expertise are less likely to contribute.

However, individuals' cognitive capital improves as they interact intensely with others and sharing the same practice and learns the "skills, knowledge, specialized discourse, and norms of the practice" (Molly McLure Wasko \& Samer Faraj, 2005, p. 41). This capital may be gained either through practical experience or through narratives told over time. Correspondingly, 
individuals with longer duration in the shared practice are likely to understand better how their knowledge is relevant, and are better able to share knowledge with other members (Kim et al., 2017; Molly McLure Wasko \& Samer Faraj, 2005).

\subsubsection{Relational Factors}

Relational factor refers to "the kind of personal relationships people have developed with each other through a history of interaction" (Nahapiet \& Ghoshal, 1998, p. 224). This concept emphasises the certain relationships that people have, such as respect and rapport, which impact their behaviour. Relational capital exists if members have a strong bonding with the network (Lewicki \& Bunker, 1996), have trust to other members (Putnam, 1995), perceive a responsibility to participate in the network (Coleman, 1990), and understand and abide by its mutual norms (Putnam, 1995). The main purpose of relational capital is to enable actions for individuals within the structure (Coleman, 1990). Through this aspect, individuals fulfil such social motives as "sociability, approval and prestige" (Nahapiet \& Ghoshal, 1998, p. 244).

Relational factor involves commitment (self-identification) to the community and reciprocity (a sense of obligation) (Nahapiet \& Ghoshal, 1998; Wasko et al., 2005). Commitment arises from the repeated interaction, and it signifies a duty or responsibility to involve oneself in future action (Coleman, 1990). Commitment to a network expresses an obligation to help others by shared membership (Wasko et al., 2005). Furthermore, it is suggested that individuals participate in the network because they perceive a moral obligation to return the favour of the network (M. M. Wasko \& Faraj, 2000). Therefore, members are eager to help other members, even strangers, as they consider it an act of duty to assist other members (Wasko et al., 2005) and only because they are part of the network with collective goal orientation (Leana III \& Van Buren, 1999).

However, while being a popular theory to examine knowledge sharing in the particular context of organisations, it is argued that social capital theory is also relevant to be applied to individual context (Molly McLure Wasko \& Samer Faraj, 2005). At the group level, social capital reflects "the affective nature and 
quality of relationships" (Wasko et al., 2005, p. 39). Meanwhile, at the individual level, social capital enables individuals' actions and reflect their access to network resources (Coleman, 1990), which explains why the social capital theory is applicable as theoretical background in this research. Therefore, in order to adjust the concept of social capital into the individual level and explain individuals' contribution within the network, Wasko et al. (2005) propose an extended framework of social capital adapted from Nahapiet and Ghoshal (1998) by adding individual motivation.

\subsubsection{Individual Motivation}

This individual motivational dimension perceives enhancing reputation and the enjoyment of helping others as the primary reasons to actively participate in specific networks (Molly McLure Wasko \& Samer Faraj, 2005). Individuals think that their contributions toward others within the network will be worth the effort and generate new values, which they expect for themselves (Hippel \& Krogh, 2003; Nahapiet \& Ghoshal, 1998). Regardless the absence of personal acquaintance or the possibility of direct reciprocity (Constant, Sproull, \& Kiesler, 1996), these expectations of personal benefits is analogous to social exchange theory, in which individuals engage in social interaction because they expect rewards such as approval, status, and respect (Blau, 1964).

Besides, to increase reputation, individuals' active participation in the network is encouraged by their enjoyment of helping others. Thus, individuals may voluntarily help other because they perceive that helping others with challenging problems is exciting and because it feels good to help other people (M. M. Wasko \& Faraj, 2000; Molly McLure Wasko \& Samer Faraj, 2005).

\subsubsection{Review of Social Capital Theory}

The social capital theory has been widely used in various academic studies in order to explain individuals' pro-social behaviours, such as collective action, community involvement, differential social achievements, and knowledge sharing in online communities (Kim et al., 2017; Wasko et al., 2005). In tourism studies, social capital theory is used to examine community development (Jones, 
2005), trust toward government and governance organisations, and resident's attitude and behaviour. Most of the studies with social capital theory as theoretical background use a quantitative method in the research. This raises criticisms that the quantitative methodology only captures the positive side of social capital, but explores less than the whole picture (Svendsen, 2006). Therefore, this research uses the qualitative methodology in order to understand better, explore and capture the host's experience from a social capital perspective.

By and large, the perspectives of social exchange and social capital theory promote the idea that the decisions of an individual to engage in an activity are driven by factors that are valued by them. While social exchange theory posits that interaction between individuals is based on the expectation of specific rewards, social capital theory relates that the individual's behaviour is structured into their relationships with their network. Therefore, as a basis for individuals' behaviour has been elaborated, the following section will present an overview of the motivation of an individual's behaviour in the collaborative consumption context.

\subsubsection{Motivation in Collaborative Consumption}

The previous chapters, presenting the perspective of social exchange and social capital theory, have provided an essential substance to understanding individuals' motivation in a certain context. Being applied to the collaborative consumption platform, it can be assumed that individuals' decisions to participate in the platform may relate to certain benefits they perceive and also their relationship to the collaborative consumption platform as a network. Therefore, in order to build a comprehensive understanding of the research, this section will now provide an overview of motivation in the collaborative consumption context.

Collaborative consumption emerged as "the peer-to-peer based activity of obtaining, giving, or sharing access to goods and services, coordinated through community-based online services" (Hamari et al., 2016, p. 2049). It gained much attention in the academic literature as it provided an alternative for 
consumption behaviour (Belk, 2014). Through the lens of supply, collaborative consumption enables individuals to monetise their resources, including possessions, skills, and time (Belk, 2014; Hamari et al., 2016; Lampinen \& Cheshire, 2016). However, this premise generalises motivation in all contexts of collaborative consumption as monetary exchange. Thus, it ignores the characteristics of non-monetary exchange platforms.

Accommodation sharing is one of the popular collaborative consumption platforms in the study of tourism. Two types of accommodation are common: monetary and non-monetary types. Of the monetary type, Airbnb is one of the favourite research subjects in the tourism study. Therefore, when it comes to motivation to host on the platform, Johnson and Neuhofer (2017); (Lampinen \& Cheshire, 2016) describe money as the dominant motive, followed by social motives. However, when questioning the decision to host in non-monetary accommodation sharing, little is known about the motivation that encourages them to host. Meanwhile, hosting in non-monetary accommodation sharing involves an unspecified return; thus the notion of sharing in this context could be seen as the sole sacrifice of the hosts.

However, individuals' decision to engage in a certain activity is not spontaneous action without prior consideration (see Chapter 2.2.2). Geiger, Horbel, and Germelmann (2017) provide insight that CouchSurfing's hosts are motivated by an altruism to their destination, so they decide to act as city guides voluntarily. In addition, Geiger et al. (2017) notes that they have needed to spend time with their guests, and learn about other culture. However, Decrop et al. (2017) also postulates that hosting in CouchSurfing provides opportunities for its members to meet like-minded people. Therefore, according to these premises, individuals share accommodation without economic benefits for personal motivation.

Meanwhile, Kim et al. (2017) see the motivation to host from a network perspective. They postulate that sharing decisions in CouchSurfing is a sense of obligation toward the network. This statement is supported by the fact that hosts of CouchSurfing might be future guests or guests that happen to be at home (Molz, 2012). Thus, these experiences arise from commitment and responsibility to involvement in the future, to help strangers based on shared membership, and 
as a moral obligation to return the favour to the network (M. M. Wasko \& Faraj, 2000).

\subsubsection{Conclusion}

Little empirical effort has been made to identify factors behind individuals' attention to sharing free accommodation. There is not much literature to explain the host's behaviour. However, it can be concluded that sharing is a personal issue, which is structured through the personal judgment of rewards and costs. Works of literature on motivation shed light on hosts' motivations in non-commercial accommodation sharing serve as influencers of social interaction between hosts and guests. Thus, values are determined by hosts' intentions to give free accommodation and collaboration between both parties.

Furthermore, it is worth noting that the notion of give and take in collaborative consumption also depends on the contextual characteristics. As in CouchSurfing, shared values and norms of the network influence the decision of individuals to participate in the network and socialize with like-minded people. Therefore, value in this context also depends on people that hosts share with.

\subsection{Value Co-creation}

The previous chapter on motivation has provided a comprehensive overview of theories and works of literature, which is essential to examine individuals' motivation to host in CouchSurfing. Having an understanding of motivation provides insight to predicting individuals' behaviour and scrutinising the social interaction between host and guest during the encounter. Thus, the literature on motivation provides a fundamental input to the process of value cocreation. Subsequently, this section will break down the literature on value cocreation to provide a theoretical foundation for the analysis of value co-creation in CouchSurfing. 


\subsubsection{Definition and Concept}

This section provides the primary substance to understand the concept of value co-creation. Firstly, an approach to value co-creation will be briefly elaborated to provide a firm foundation for the concept. Secondly, the general definition and concept of value co-creation will be discussed, followed by a brief explanation of the components that build upon the concept of value co-creation.

The concept of value co-creation comes from the dualism of value creation, namely goods-dominant logic and the service-dominant logic (S. L. Vargo et al., 2008). Goods-dominant logic or traditional value creation focuses on the company's output and price as the representative of value (Prahalad \& Ramaswamy, 2004; S. L. Vargo et al., 2008). The focus of this view is making and distributing tangible goods to be sold to customers. Therefore, it is clear that in this view, the company performs as the sole value creator (Prahalad \& Ramaswamy, 2004; S. L. Vargo et al., 2008). Meanwhile, service-dominant logic views that all exchanges are based on service, which is defined as "the application of specialised competencies (knowledge and skills) through deeds, processes, and performances for the benefit of another entity or the entity itself" (S. L. Vargo et al., 2008, p. 2). From the definition, it can be implied that knowledge and skills are central to value co-creation as resources that take action in the process of value creation. S. Vargo and Lusch (2004) use the term "operant resource" to refer to knowledge and skills, and "operand resource" (such as goods, spaces) to label resources on which an act is performed by operant resources to produce an effect. The premise of service-dominant logic then serves the basic foundation for value co-creation, a "joint creation of value by the company and the customer" (Prahalad \& Ramaswamy, 2004, p. 8).

Further, S. L. Vargo et al. (2008) postulates that in the notion of value cocreation, a value will emerge and be determined through integration and application of operant and (sometimes) operand resources. While the operant resource acts as the primary key, which performs on other resources, the operand resource is a tool that enables the operant resource to derive value (S. L. Vargo et al., 2008). Meanwhile, this also implies that value co-creation does not perceive value as pre-existing (Campos et al., 2015; S. L. Vargo et al., 2008) but 
something that customers and manage in their consumption context (S. L. Vargo et al., 2008).

Value co-creation sees the customer as an active actor that determines and creates value in their values creating process (Campos et al., 2015; Prahalad \& Ramaswamy, 2004; Shaw et al., 2011; S. L. Vargo et al., 2008). By this premise, experience and perception are crucial in determining the value and therefore value co-creation is always personal, experiential, and related to specific context and rich in meaning (Lusch \& Vargo, 2006).

As the definition and the general concept of value co-creation has been elaborated, a contextual aspect of value co-creation is required. To provide a context for value co-creation in the thesis, the following section will now discuss how the literature posits value co-creation in the tourism and hospitality study.

\subsubsection{Value Co-Creation in Hospitality Context}

As the general concept and definition of value co-creation has been described in the previous section, the literature of value co-creation in the context of hospitality will now be examined. This chapter will provide a contextual application of value co-creation and elaborate on how the concept has developed. Hence, the definition of co-creation in the study of tourism will first be briefly delineated, followed by the literature reviewed from the perspective of tourist and business.

Co-creation in tourism studies can be defined as "a process of interrelated interactions and activities that connects the tourist and other actors, and experiences are the context in which those interactions and activities occur" (Campos et al., 2016, p. 1311). The definition implies that co-creation is a context, wherein tourist engages with other actors in the destination through interactions and active participation. The co-created value thus emerges as a result from interaction, active participation, and collaborative effort that build up the experience (Agrawal \& Rahman, 2015; Saarijärvi, Kannan, \& Kuusela, 2013; S. Vargo \& Lusch, 2004; S. L. Vargo et al., 2008).

Value co-creation has been applied widely in tourism context, including in hospitality studies (Bharwani \& Jauhari, 2013; Cabiddu et al., 2013; P. Chathoth 
et al., 2013; P. K. Chathoth, Ungson, Harrington, \& Chan, 2016; Shaw et al., 2011). However, reviewing the literature on value co-creation should be seen from two perspectives, from tourist and business perspectives. By thus, it provides a comprehensive understanding of value co-creation from two different perspectives of value creators or beneficiaries.

\subsubsection{Value Co-creation from the Tourist's Perspective}

Instead of being passive and consuming tourism activities as the way of seeing (Urry, 2002), tourists take an essential role in value co-creation as a value co-creator (Rihova, Buhalis, Moital, \& Gouthro, 2015). The literature depicts the

tourist as an actor, who contributes to "some aspect, phase or the overall tourism experience" (Campos et al., 2015, p. 19), by engaging in on-site personal experience through active participation and interaction with other subjects during the experience. Therefore, the value appears as the meaning tourists derive from the experience they design, create and consume (Bharwani \& Jauhari, 2013; Campos et al., 2016).

Campos et al. (2015) depicts the relationship between actors during the encounters (on-site) on the experiencescape framework (see Figure 2.1). In the framework of on-site value co-creation, a tourist is a central actor, who engages mentally and physically in active participation and interaction with other subjects and environments at the destination. Values in the interactive relationship then emerge as psychological states and process such as sensations, perceptions, images, feeling, and emotion. Aside from that, the experiencescape integrates dimensions, namely physical aspects, social actors and organisational features in the co-creation experience. Being called as influencers, these dimensions are resources, which manage and harness by the host to influence the way the tourist lives the experience. 
Figure 2.1 Experiencescape

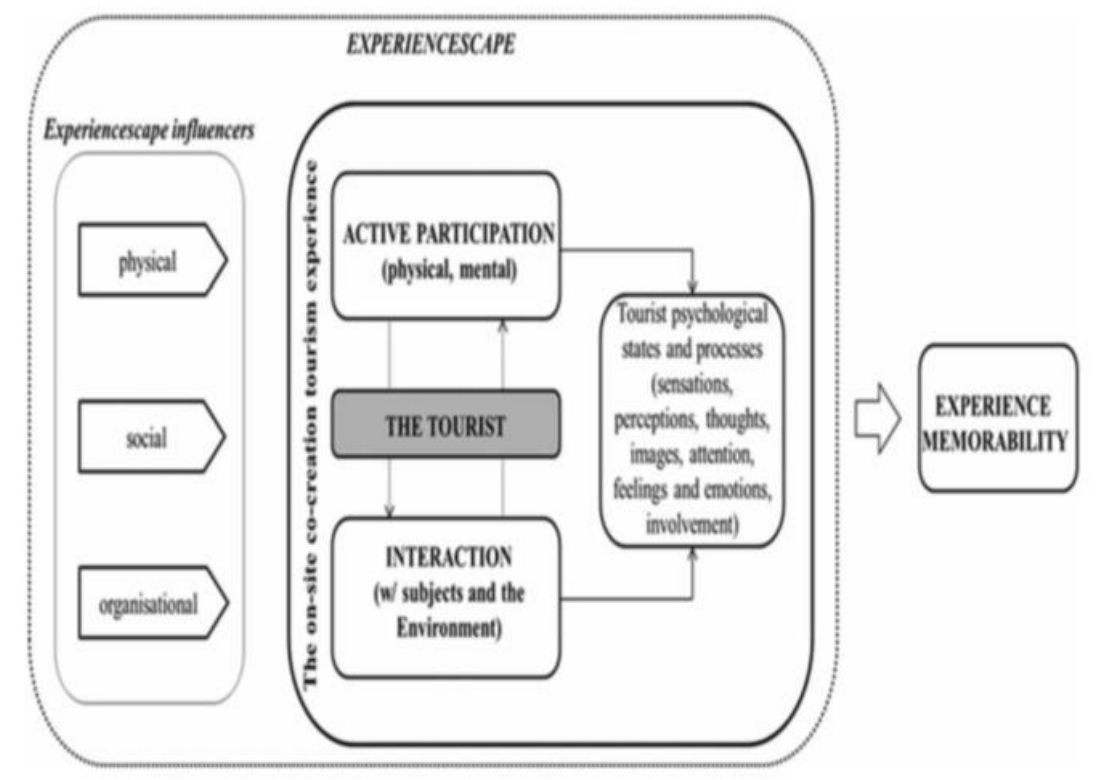

Source: Campos et al. (2015)

\subsubsection{Value Co-creation from Tourism Service Provider's Perspective}

Value co-creation in the hospitality industry from the perspective of guests provides insight into the process of value co-creation and which resources exert influence. Meanwhile, this study takes the perspective of host in analysing value co-creation. Therefore, value co-creation from the perspective of a business provider will now be presented as a counter perspective and provide a theoretical perspective for the study.

From the business perspective, the literature usually adopts an industry perspective, which perceives tourism as a system comprised of its people and the tourist, determined by a high level of interaction (Neuhofer, Buhalis, \& Ladkin, 2012). Therefore, this industry perspective considers interactions as elements of the tourist experience that need to be "carefully designed, integrated and managed to ensure an emotional connection, loyalty and satisfaction" (Lugosi \& Walls, 2013, p. 52). Further, from the business point of view, cocreation is a business orientation and strategy toward competitiveness, which relates to management and marketing principle (Campos et al., 2015). As a business orientation, tourism service providers involve tourists in the joint value creation in creating new products and services (Bharwani \& Jauhari, 2013; 
Cabiddu et al., 2013; Campos et al., 2015; Johnson \& Neuhofer, 2017). Therefore, to successfully conduct this function, competencies and behaviour from hospitality employees are crucial in determining and deriving values. Similarly, as a way toward competitiveness, co-creation is an innovation to overcome the shifts in tourist behaviour (P. K. Chathoth et al., 2016; Johnson \& Neuhofer, 2017) by engaging customers on an emotional level and enhancing their satisfaction. In order to do that, hospitality organisation must be able to provide "holistic experiential service offerings" (Bharwani \& Jauhari, 2013, p. 824), which engage each customer on a personal and emotional level.

Reviewing the literature, to derive value from co-creation experiences, tourism service providers must shift their role as 'service providers' into 'experience providers' (Bharwani \& Jauhari, 2013). Therefore, they are required to harness and manage their resources to deliver memorable experiences for tourists, by developing hospitality competencies (Bharwani \& Jauhari, 2013) and building social connections (P. K. Chathoth et al., 2016; Shaw et al., 2011). Developing hospitality competencies relates to the needs of connecting emotionally with tourists and achieving a significant level of engagement by using a set of integrated skills required to conduct an excellent quality of interaction (see Figure 2.2). In order to achieve that, Bharwani and Jauhari (2013) argue that aside from technical ability, the employee in the hospitality industry is required to have hospitality intelligence to deliver an interactive social interaction. Thus, Bharwani and Jauhari (2013) propose a framework of hospitality intelligence (Figure 2.2) to illustrate a set of required skills, which enable an employee to lift guest experience. 


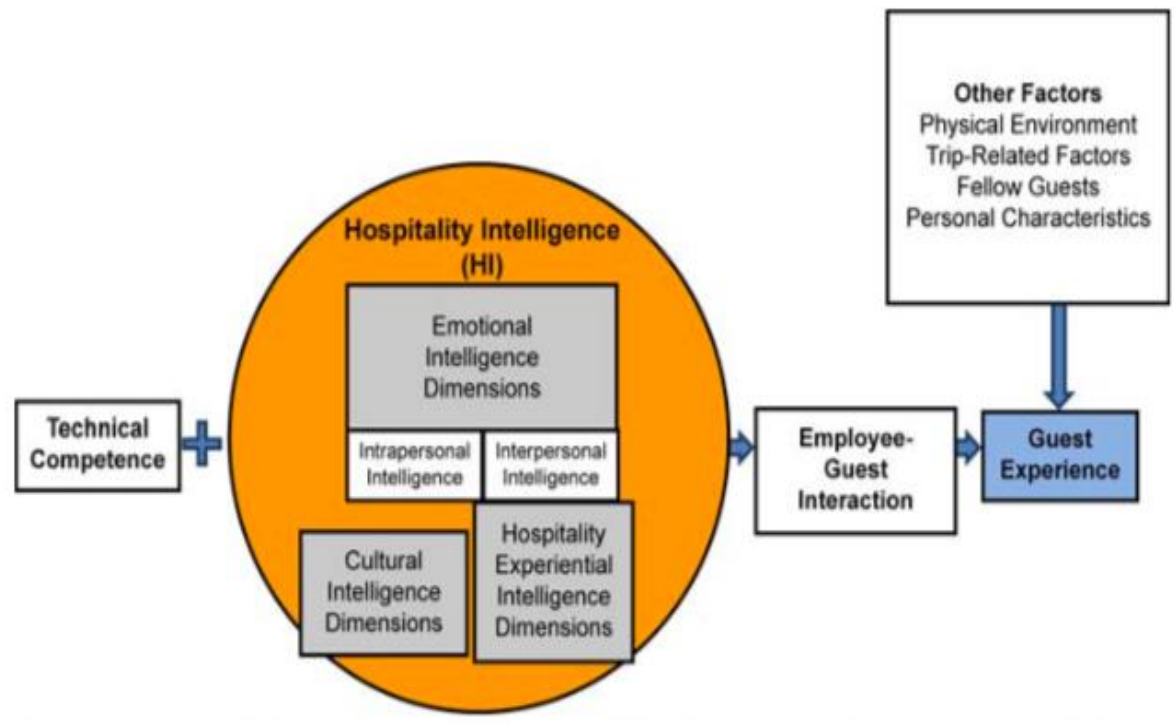

Source: Bharwani and Jauhari (2013)

The first skill under hospitality intelligence is emotional intelligence, the ability to use emotions as guidance for interpersonal effectiveness (Bharwani \& Jauhari, 2013) and manage emotion in "a culture-neutral context" (Bharwani \& Jauhari, 2013, p. 832). This intelligence comprises intrapersonal intelligence and interpersonal intelligence. While intrapersonal relates to dealing with one's self, interpersonal competencies deal with managing relationships with others. Aside from emotional intelligence, there is cultural intelligence, which refers to the flexibility of individuals to function and interact effectively in an intercultural setting (Bharwani \& Jauhari, 2013). This competency provides individuals with insights and capabilities to deal with multi-cultural situations and engage in cross-cultural encounters. Having emotional and cultural intelligence, an employee should also be equipped with hospitality experiential intelligence, the ability of employees to deal with problems or new situation by using knowledge and skills in hospitality. Bharwani and Jauhari (2013) explain that this intelligence is related to the ability to personalise and enrich guest experiences and make them central to the consumption process; creativity and innovation, and also the professional behaviour, like respecting guest privacy or having a genuinely welcoming attitude. 
In addition to developing hospitality competencies, another resource needed for the tourism service providers to co-create value is building a social connection with a consumer. This strategy requires a hospitality organisation to proactively and effectively improve customer/employee interface from a simple interaction to a positive, memorable experience (Bharwani \& Jauhari, 2013; P. K. Chathoth et al., 2016; Shaw et al., 2011). This can be done through utilising the customer as an operant resource (P. K. Chathoth et al., 2016), meaning using customer's skill and knowledge in the process of value co-creation (Shaw et al., 2011); creating a 'touch point', wherein customer and employee interface occurs, and having social interaction (P. K. Chathoth et al., 2016), and accessing consumer information as a strategy to cater to guests with personalised offerings (Bharwani \& Jauhari, 2013; Prahalad \& Ramaswamy, 2004). Customers will engage with the company only if they are convinced that firms have the willingness to engage with them in order to create positive experiences and value (Bharwani \& Jauhari, 2013). If they are not convinced, they will not share any personal information, and thus, customer experiences cannot be maximised. Therefore, hospitality organisations should manage and consider this "paradox of customer information, engagement and experiences" (Bharwani \& Jauhari, 2013 , p. 230) carefully to be successful in value.

\subsubsection{Review on Value Co-creation in a Hospitality Context}

Interaction and active participation between actors is the primary requirement to derive value from co-creation experiences (Campos et al., 2015; Prahalad \& Ramaswamy, 2004; S. L. Vargo et al., 2008). However, to holistically understand the application of value co-creation in the hospitality industry, two perspectives of beneficiaries are required. From the perspective of guests, cocreation experience is a system in which a tourist engages mentally, physically, and cognitively with other actors during their tourism experience (Campos et al., 2015). Thus, the value is the psychological states and processes, which impact the degree of memorability of their experience. Meanwhile, from the perspective of the hospitality industry, co-creation is a business orientation toward competitiveness (Prahalad \& Ramaswamy, 2004; Shaw et al., 2011; S. L. Vargo et 
al., 2008). Businesses see co-creation as a step toward competitiveness and a solution to the challenge of shifting tourist behaviour, by developing a set of skills to elevate consumers in a higher degree of engagements (Bharwani \& Jauhari, 2013).

However, existing literature shows a limitation in the way they use popular high-end hotels as case studies. Concerning this thesis, information provided by the existing literature neglects accommodation sharing platforms, which have a specific and distinct nature of interaction, and whose consumer have distinct characteristics. Therefore, in order to fill the gap, the following section will present the literature of value co-creation and relate it to a collaborative consumption context.

\subsubsection{Value Co-Creation in Collaborative Consumption}

Extant literature of value co-creation puts the focus on the hotel as the empirical context and ignores different characteristics of accommodation. Therefore, research on value co-creation in the hospitality industry has a gap in understanding of how values are co-created in collaborative consumption accommodation platforms. This thesis is looking for value co-creation in the context of non-monetary accommodation sharing. Apparently, the reviewed literature is not adequate to portray the relationship between host and guest within the context because of the different nature of the interaction. Therefore, in order to provide contextual value co-creation background, this section will now elaborate value co-creation on collaborative consumption platform.

Compared to conventional accommodation providers such as hotels, accommodation sharing has a different kind of interaction between host and guest. It is argued that the relationship between host and guest in the collaborative consumption platform is more intimate and authentic (Molz, 2012; Pietilä, 2011; Schuckert et al., 2017). This intimacy and authenticity are reflected in the way accommodation sharing provides access to the local community (Guttentag, 2015) to learn the norms, behaviours, habit and cultures of the destination (Holtz, 2014; Schuckert et al., 2017). However, unlike conventional accommodation services, guests in accommodation sharing have access to 
"homely amenities" (Johnson \& Neuhofer, 2017, p. 2364) such as the kitchen and living room. Guests also have a direct encounter with the host, and they have the opportunity to conduct social practices during their stay. Past research on value co-creation in collaborative consumption enumerate these social practices manifest in activities such as cooking and cleaning at home, cultural learning (Johnson \& Neuhofer, 2017; Schuckert et al., 2017) and doing activities beyond usual tourist maps (Molz, 2012).

These activities between host and guest in accommodation sharing confirm the importance of joint value creation (Guttentag, 2015; Johnson \& Neuhofer, 2017; Kim et al., 2017; Molz, 2012; Schuckert et al., 2017). Being located within the local community provides collaborative consumption platform with a value proposition, the access to authenticity. Empirically studied from the perspective of a guest, these studies emphasise the importance of host as an operant resource (S. Vargo \& Lusch, 2004) with skills and knowledge to directly assist guests in living their experience to add value proposition. Also, the studies reveal that value will emerge as hosts integrate their skills and knowledge (operant resource) with physical attributes (operand resource) to deliver excellent service to guests. Through this, hosts become key to influencing social practices, in which their ability as the integrator of resources will define the quality of interactions and consequently value determined from the interactions.

While research on value co-creation has gained attention in the study of tourism, not much literature explains the process of value co-creation in collaborative consumption platform. Moreover, the extant literature on value cocreation in collaborative consumption platform is empirically examined in the context of monetary accommodation sharing and through the perspective of the guest. Therefore, this raises a question as to if the findings apply to the context of non-monetary accommodation sharing. With little is known about the practice of value co-creation in non-monetary accommodation sharing contexts; thus, how and what values are co-created in the context remain an assumption.

However, the literature on motivation and value co-creation has been elaborated and reviewed to provide a theoretical foundation in conducting the thesis. In order to provide further investigation, it is essential to set out the key concepts and relationships between these concepts in motivation and value co- 
creation practices in non-monetary accommodation sharing. Hence, the conceptual framework will be presented in the following section, as integrated literature and a basis for the data collection, explaining how the analysis will be undertaken.

\subsubsection{Conceptual Framework}

While the literature on motivation and value co-creation has been elaborated upon, this section will present a conceptual framework in order to identify and bound knowledge from the reviewed literature. The purpose of the conceptual framework is to illustrate the focus of the research, from which the data will be collected, and analysis will be conducted.

Reviewing the literature, the study on human behaviour provides insight into individuals' motivation to engage in certain activities (see Chapter 2.2.2; Chapter 2.2.3). Individuals decide to enter social interactions as they are after something valued to them, and they are affected by their experience and relationship to the network. Meanwhile, in the light of value co-creation, values result as integration and application of a set of resources in social interaction (S. L. Vargo et al., 2008). While motivation is reflected in the way hosts conduct social interaction, it can be inferred that value is an illustration of the motivation.

The conceptual framework of social interaction between host and guest in CouchSurfing displayed in Figure 2.3 consists of four parts, namely motivation, value co-creation practice, resource, and value outcome. The first part of the framework is motivation. This part is the beginning of the entire process, in which hosts determine their intention to host on CouchSurfing. Social exchange theory provides a basic understanding of individuals' decision to host as it relates to certain rewards: love, status, information, goods, money, or services. Meanwhile, this decision is also influenced by their previous experience/ exchange, which is perceived by the individuals as an obligation to return the favour, a sense of responsibility for past actions. In conjunction with social exchange theory, the social capital theory postulates that individuals' decisions to provide non-monetary accommodation sharing relate to their relationship with CouchSurfing as a network. It postulates that individual's relationship with 
the network and how they perceive other members determines their sharing behaviour. Further, little is known about the motivation of individuals to participate in collaborative consumption platforms, especially in noncommercial accommodation sharing. As the current studies of motivation in collaborative consumption examine from the perspective of guests and monetary accommodation sharing, it remains a question if the findings apply to the context of non-monetary accommodation sharing.

The second part of the framework is value co-creation practice. In this part, the host is actively having social interactions with the guest. This stage is the process of determining value, in which hosts are trying to practice their motivation. However, to be successful in deriving value from social interaction, hosts must integrate and apply their resources, which influence the way they conduct social interaction. Grouped under the third part, these resources involve social and physical resources. Social resources are related to the host as a social actor who possesses skills and knowledge. These might include communication skills, cultural intelligence, hosting experience, and so on. On the other hand, the physical resource relates to the physical aspect of the interaction (such as space, cleanliness, environment, and so on). In the framework, the social resource is appointed to the physical resource, which illustrates that social resources take action or perform on physical resources. This also implies that these resources are not separate entities but need to be integrated to take action successfully. The last part of the framework is value outcome. As an outcome, this part is the result of the host/guest relationship in part 2 and 3. Besides, this part is interrelated to motivation, a reflection of the rewards expected by individuals.

However, with the limited works of literature of the study, it is unclear which type of interactions characterise the host/guest relationship in CouchSurfing and which resources are required and exert influence on emergent values. While the literature on value co-creation in the hospitality context mostly focuses on monetary accommodation and mainly through the perspective of a guest, it also remains questionable to describe the process of value co-creation and thus value resulting from the interaction.

To sum up, the conceptual framework provides a wide range of information to examine value co-creation in non-commercial accommodation 
sharing. While the gap is an illustrative representation of the extant literature, it also highlights which data will be explored in data collection. The conceptual framework serves as guidance to design research instruments and methodology, which will be discussed in the following chapter.

Figure 2.3 Conceptual Framework for Value Co-creation in CouchSurfing

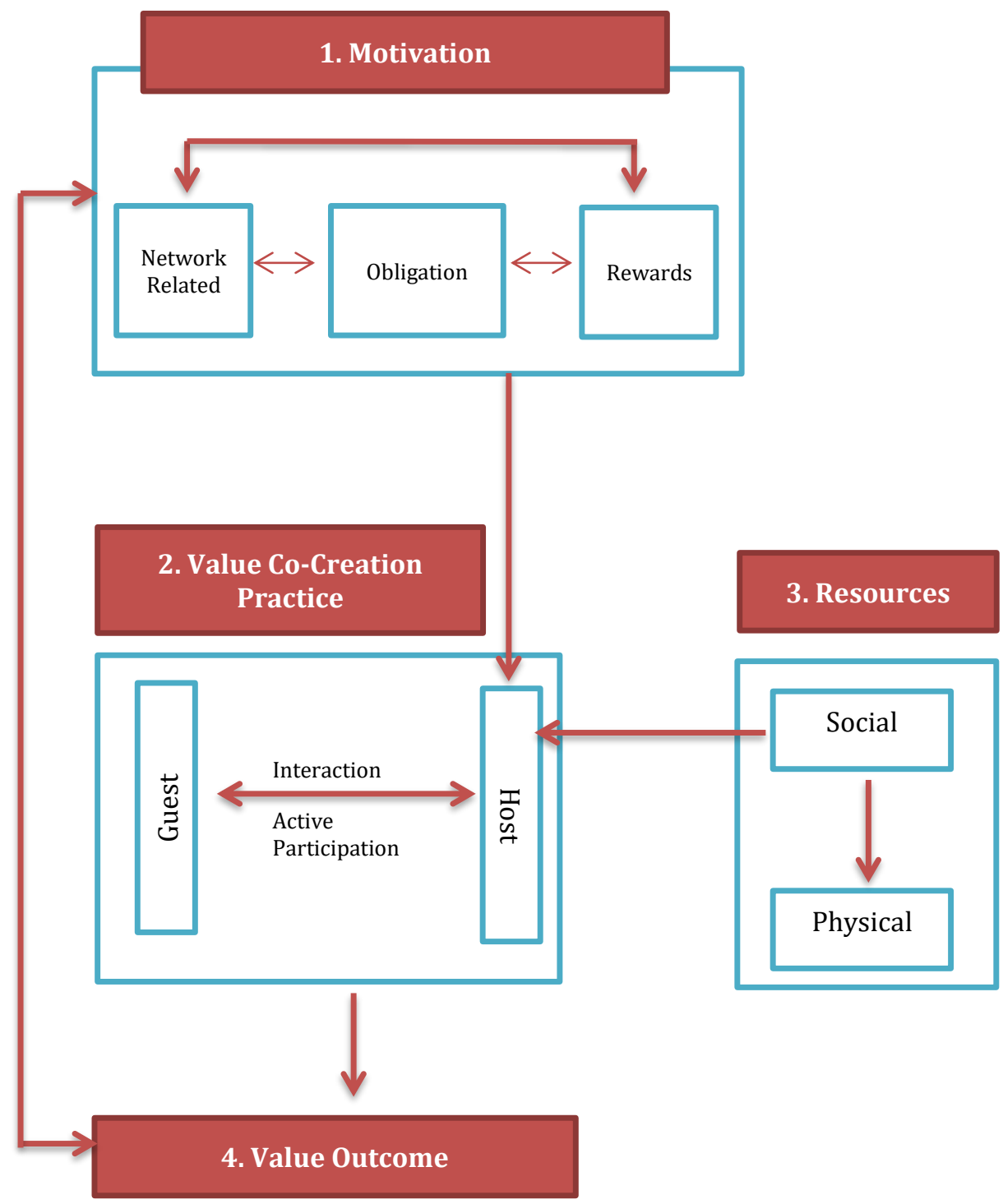




\section{Methodology}

\subsection{Introduction}

The previous chapter has provided an overview of literature in the motivation and value co-creation, followed by a conceptual framework. Serving as integrated literature, the conceptual framework also functions as the basis for the methodology of this research. Regarding that, this chapter will now elaborate on the steps performed in conducting the research. First, the research questions will be revisited, and the data needed for each question will be detailed. This step is essential to ensuring that issues carried by each research question are accommodated in the data collection. The following section is the research paradigm, which will elaborate direction of the research. Subsequently, the research design will be presented, covering a set of the methodology in collecting the data. Finally, data analysis will describe the techniques of analysis, followed by an analytical framework, which serve as a guide to present the findings.

\subsection{Research Questions}

The conceptual framework presented in Figure 2.3 provides an overview of the literature in motivation and value co-creation in non-monetary accommodation sharing. However, the reviewed literature shows that the current study does not provide an adequate theoretical foundation to examine motivation and value co-creation in the context of non-monetary accommodation sharing. Therefore, some information is required to meet the objectives of the research and provide an answer to the research questions:

\section{1) What is the motivation of Indonesian CouchSurfing members to host?}

This research question concerns contributing factors that encourage individuals to host in CouchSurfing. Reviewed literature provides insight that individuals' decision to host may relate to certain rewards and the need to return the favour they have received in the past. Further, the literature suggests that motivation to host may also relate to 
individuals' relationship to a specific network, which generates a sense of responsibility to help others based on shared membership. While reviewed literature informs the possible reasons to host, it is important to note that these studies are not correctly applied to non-monetary accommodation sharing. Consequently, the particular reason for individuals to host in CouchSurfing is unclear. Therefore, in order to provide an answer to these questions, the following issues need to be considered:

- What rewards are hosts after, and why?

- Do they have experience as a guest from CouchSurfing platform? How do they perceive that?

- How are embedded individuals in the network? How do they perceive CouchSurfing and its members?

\section{2) Through what practices are values co-created between host and guest in a CouchSurfing context?}

While research on value co-creation in hospitality has its focus on hotels and monetary accommodation sharing as its empirical study, it is important to note that there is financial involvement in the exchange. Meanwhile, the context of this research is non-monetary accommodation sharing, which develops different characteristics of exchange than monetary platforms. Therefore, it is sufficient to say that the current literature on value co-creation is not adequate to examine the process of value co-creation in non-monetary accommodation sharing. Concerning the theoretical background, it is arguable whether the concept of value cocreation empirically studied in monetary accommodation platforms applies to non-monetary ones. Consequently, when it comes to the relationship between host and guest in non-commercial accommodation sharing, little is known about how and why that occurs.

In order to fill the gap, research question 2 explores how interactions occur between hosts and guest in non-monetary accommodation sharing contexts. Issues that need to be addressed are as follows: 
- How do the members see themselves as hosts? What is their role?

- How do hosts interact with guests? Which interactions occur?

- How does motivation influence interaction?

\section{3) Which resources needed for these social practices?}

Research on value co-creation postulates that social practice between actors is affected by the ability of the actors to integrate their resources (Campos et al., 2015; P. K. Chathoth et al., 2016; S. L. Vargo et al., 2008). While the literature delineates the resources to value cocreation practice as social and physical resources (S. L. Vargo et al., 2008), which resources exert their influence in the interactions of non-monetary accommodation sharing are questionable. Moreover, how hosts utilise these resources, and to what extent these resources influence social interaction, is unknown. Therefore, research question 3 explores the resources needed to conduct social interaction. This research question will address the following issues:

- Which resources belong to the social and physical category?

- How do social and physical resources utilised by hosts in conducting social interaction?

- How do these resources affect the interaction between host and guest?

\section{4) What values emerge as outcomes for hosting in CouchSurfing?}

Reviewed literature postulates that in co-creation, value is always co-created through the integration of a set of resources to value cocreation practice (Prahalad \& Ramaswamy, 2004; S. L. Vargo et al., 2008). As the literature postulates that motivation will affect individuals' behaviour in social interaction, it is assumed that motivation will have interrelation with value. Meanwhile, little has emerged from the literature concerning value as the outcome of value co-creation in non-monetary accommodation sharing. Consequently, research question 4 addresses the following issues: 
- What values are co-created?

- How do hosts compare values to motivations?

\subsection{Research Paradigm}

This research paradigm will comprise the guidelines and principles in conducting the research in this thesis, and determines the methods and techniques used in the research design.

The research paradigm adopted for this study is social constructionism. In this view, "the construction of the social world is seen as occurring through a shared process of communication and social interaction" (Hammersley, 2013, p. 34). This paradigm sees that individuals seek to understand the world they live in and develop subjective meanings that correspond to their experiences (Creswell, 2013). These meanings are varied and multiple (Creswell, 2013) and they are not merely imprinted within individuals. Instead, these meanings are formed through interaction with others. Knowledge then is perceived as something that people create and enact together (Burr, 2015; Creswell, 2013; Hammersley, 2013).

Further, social constructionism concerns social practices engaged by individuals and their interaction with others (Burr, 2015; Creswell, 2013; Hammersley, 2013). It focuses on the dynamic of the social interaction (Burr, 2015), what is going on between people in their everyday lives, and the process of the phenomena (Burr, 2015; Creswell, 2013). Social constructionism insists on looking into objects behind these phenomena and how their characters are generated. Therefore, social constructionism leads researchers to examine the complexity of views rather than narrow their meaning into a few categories or ideas. The goal of this research, then, is to consider as much as possible about participants' views of the situation (Au, 1998; Creswell, 2013).

In practice, social constructionism regards objectivity as an impossibility (Burr, 2015; Creswell, 2013). Researchers are involved in the processes whereby social phenomena are constructed, and they cannot escape this (Creswell, 2013). In other words, researchers acknowledge that their background shapes their interpretation (Creswell, 2013), and they posit themselves in the research to 
admit how their interpretation flows from their own personal, cultural, and historical experiences (Burr, 2015; Creswell, 2013). In other words, researchers interpret their findings, shaped by their own experiences and background. Therefore, the researcher must recognise their intrinsic involvement in the research process and acknowledge this part in the findings (Creswell, 2013). The researcher must also view the research as a co-production between themselves and the researched (Au, 1998; Burr, 2015; Creswell, 2013). The researcher's focus, then, is to "make sense (or interpret) the meanings others have about the world" (Creswell, 2013, p. 21).

Social constructionism is adopted in this study in several ways. Generally, it supports the focus of the study in examining the social interaction between host and guest. It explores how this social interaction is constructed through the perspective of hosts by investigating motivation and what resources hosts must make the social interaction valuable. Research question 1 questions the motivation behind the hosts' decision and social interactions. From the social constructionist perspective, the inquiry lies in factors contributed to provide free accommodation and engage in social interaction. It does not only focus on the rewards but also put the inquiry on hosts' experience and their embeddedness in the CouchSurfing as contributing factors.

Moreover, in research question 2, 3 and 4, this thesis questions how the practice of social interaction is conducted. Social constructionism examines this in the way hosts and guests make interactions and how the contributing factors shape these interactions. Lastly, the social constructionism paradigm reflects on how collaboration between host and guest generate values, specifically for hosts. It questions values co-created between the actors and how values interlink with hosts' motivation.

Overall, the social constructionism paradigm supports the focus of the study in the way it provides perspective to answer the research questions. From the methodological approach, the nature of social constructionism leads this thesis to adapt qualitative research. It is considered the most suitable method of enquiry to gain linguistic and textual data to provide space for the respondents in narrating their experiences (Burr, 2015; Creswell, 2013). By thus, qualitative methods are considered less likely to decontextualize the experience and 
account of respondents (Burr, 2015). Subsequently, the following sub-chapter will present steps undertaken to collect the data.

\subsection{Research Design}

As the research paradigm has been established, the following section will discuss in detail the implementation of the paradigm into the research design, which involves qualitative research as a method of inquiry, semi-structured interview, interview guidelines, and sampling design.

\subsubsection{Qualitative Research}

Social constructionism is associated with qualitative research in the method of enquiry, in the way it accommodates linguistic and textual data for the respondents to narrate their experiences (Burr, 2015; Creswell, 2013). Qualitative research aims to discover "the patterns that emerge after close observation, careful documentation and thoughtful analysis" (Cavana, Delahaye, \& Sekaran, 2001, p. 135). It is argued that until these patterns are identified, the quantitative proof of the causal nature of the variables cannot be investigated (Cavana et al., 2001). Further, qualitative research investigates "people's value, interpretative schemes, mind maps, belief systems and rules of living" so that participants' reality can be comprehended (Cavana et al., 2001, p. 34), to discover how individuals construct meaning in their contextual setting (Hammersley, 2013). Therefore, qualitative research sees the world as complex and interconnected, and it takes a perspectival view rather than taking an objective position in understanding reality, meaning that reality is socially and subjectively interpreted instead of being objectively determined (Cavana et al., 2001; Hammersley, 2013; Myers, 2009).

This study is looking into social interaction between host and guest in non-commercial accommodation sharing setting through investigating motivation and value co-creation. Therefore, a method, which allows individuals to narrate and explain the way they live their experience in the context was required. This scheme could only be established through direct communication with hosts to let them reveal the whole experience. Hence, qualitative research was adopted to explore a complex and detailed understanding of individuals' 
experience.

\subsubsection{Semi-Structured Interviews}

While qualitative interview has been established as a method to collect data, this section will now present the approach to conduct the interview. Taking constructionism as a paradigm to conduct the research, the researcher should address the process of interaction among individuals (Creswell, 2013) by listening carefully to what people say or do in their live setting. Therefore, the approach to the participants was established through the more open-ended the questions in order to provide them with space or 'freedom' to narrate their story (Cavana et al., 2001; Galletta, 2013; Hammersley, 2013; Myers, 2009; Sekaran, 2010).

Regarding the aim of the study, little is known about the practice of value co-creation in non-commercial accommodation sharing. Although the concept of value co-creation has been widely studied and has gained much attention in tourism and hospitality studies, it does not adequately explain social interaction in non-commercial accommodation sharing and how actors derive values in practice. Therefore, a scheme, which allowed the researcher to obtain in-depth information on social interaction and value co-creation, and ensures the participants describe their experiences comfortably and openly, was required. Thus, considering the paradigm and the need for the study, a semi-structured interview was adopted as an approach to the participants.

The semi-structured interview is a hybrid method of an unstructured and structured interview (Cavana et al., 2001; Galletta, 2013; Sekaran, 2010). It is considered a suitable approach to interview as it "reflects variation in its use of questions, prompts, and accompanying tools and resources to draw participants more fully into the topic" (Galletta, 2013, p. 45). It involves both open-ended and more theoretically driven questions, which allows the researcher to elicit data grounded in the experience of the participant as well as data guided by the existing study (Cavana et al., 2001; Galletta, 2013). The open-ended questions allow the researcher to decide what variables or information should need further, in-depth investigation as some preliminary issues arise (Cavana et al., 2001; Sekaran, 2010) but also are considered theoretically to more reflect the 
world of the interviewee (Cavana et al., 2001). Besides, semi-structured interviews are conducted with specific individuals who are invited to participate because of their knowledge and/or expertise in a particular field.

However, although the semi-structured interviews are designed more closely on the unstructured type of interview than on the structured one (Minichiello, 2008) in the way there are no fixed wording or order of the questions (Minichiello, 2008), the focus of the interview is still tied to the content raised by the research questions (Cavana et al., 2001; Galletta, 2013; Minichiello, 2008). Therefore, by using the semi-structured interview, the participants were guided by structure based on the content of the thesis. The interview thus did not wander away from the research object, but at the same time, did provide the flexibility for participants to narrate their experience.

\subsubsection{Interview Guide}

In the semi-structured interview, guidelines are essential to allow the engagement of the participants (Galletta, 2013) and to let the interview attach to the content of the research (Cavana et al., 2001; Myers, 2009; Sekaran, 2010). Therefore, Galletta (2013) argued that the interview guidelines (see Appendix E) should be carefully designed in order to accommodate the purpose of the study.

In the opening segment of the interview, the participants are asked some open-ended questions, with the purpose to create space for them to narrate their experience (Galletta, 2013). Initially, the participants were asked some openended questions about CouchSurfing and their experience in the CouchSurfing. These questions were on their tenure/duration of their membership in CouchSurfing, and the reasons for them to become members and provide free accommodation. In the next segment, the participants were required to explore deeper their experience in CouchSurfing, especially on the interaction with the guest. They were asked to describe themselves as a host and narrate their latest experience of hosting, followed by questions on positive and negative experiences in hosting. Through this, the researcher sought to understand their expectations toward hosting activity or guests. Also, through these two 
questions, it could be seen how they constructed the meaning of hosting and interaction with guest.

In the last segment, the researcher revisited the previous two segments and questioned the participants on any topics needing to be further explored. While the participants could add information, they were informed on the further research process and how they could provide feedback or withdraw from the research.

\section{Table 3.1 Interview Guidelines Mapping}

\begin{tabular}{|ll|}
\multicolumn{1}{|c|}{ Research Question } & \multicolumn{1}{c|}{ Questions } \\
\hline RQ 1 & How long have you been a \\
& CouchSurfing member? \\
\hline RQ 1 & Why are you a CouchSurfing member? \\
\hline RQ 1; RQ 4 & Have you had any experience as a \\
& guest in CouchSurfing? \\
\hline RQ 2; RQ 3 & Why do you want to give free \\
& accommodation? What does it mean \\
& to you? \\
\hline RQ1; RQ2; RQ 3; RQ 4 & Can you describe yourself as a host? \\
\hline RQ1; RQ2; RQ 3; RQ 4 & Could you tell me your latest hosting \\
& experience in CouchSurfing? \\
& Can you share some memorable \\
& experience with a guest during their \\
& stay? \\
\hline RQ1; RQ2; RQ 3; RQ 4 & Can you share any experience that \\
& you do not perceive in such a positive \\
& way? \\
\hline
\end{tabular}

\subsubsection{Sampling}

Conducting semi-structured interviews involves inviting people with certain knowledge or expertise in a particular field to be the participants 
(Cavana et al., 2001; Myers, 2009; Sekaran, 2010). Therefore, determining the sample for research is essential as by studying the sample and understanding its characteristics, it would be possible to generalise the properties or characteristics to the population elements (Cavana et al., 2001; Sekaran, 2010). The purpose of sampling for research is to identify, select and obtain access to the relevant data sources, which may either belong to or related with the broader population (Sekaran, 2010). The sampling strategy needs to construct either a representative sample of a chosen population or sample that may represent a situation, get insight, or collect information about a particular event (Sekaran, 2010). Consequently, defining the right sample results in a more reliable result.

This research targets hosts of CouchSurfing in Indonesia. With a total number of 267, 886 hosts in Indonesia (Couchsurfing, 2018), it was impossible to collect the data from the whole population of CouchSurfing hosts. Therefore, in the process of selecting a sufficient number of hosts to be interviewed, some characteristics were applied as the sampling frame. The research took hosts with characteristics as follows: Indonesian, host of CouchSurfing, residing in Java and Bali, have at least one reference from guest, and active as members within the last six months. These sampling frames were applied through filters in CouchSurfing host searching feature.

As the research took simple random sampling design in collecting the participants, every element in the population within the sample frame had a known and equal chance of being chosen as subjects in the sample (Cavana et al., 2001). Therefore, every host with the matching characteristics was contacted, and 20 participants were selected for the interview. The selection was based on the 20 first participants to contact the researcher.

\subsubsection{Data Collection}

The process of selecting the participants was conducted through the CouchSurfing platform. They were filtered based on their nationality, residency, active membership, and reference. However, during the selection process, using only 'having reference' filter did not accurately select participants with the 
hosting experience, as the platform did not differentiate reference as a host and as a guest. Hence, 'host experience' filter was added.

Upon the first contact, the potential participants were contacted through CouchSurfing's message feature along with the link, which directed them to information on the research and the consent form. If the members agreed to take part in the research, they could contact the researcher by replying the message or through WhatsApp application. By then, the researcher and the participants could arrange time and place for the interview.

The responding members were those from Bandung, Jogjakarta, and Bali. Therefore, the interviews were located in these cities and taken place in public spaces such as café or library, which were convenient to the participants. As all participants are Indonesian-native speaker, the interviews were conducted in Indonesian to uncover deeper information and to provide the participants with the flexibility to narrate their story. Mostly, the interviews were conducted in the evening, after office hours, or on the weekend. Before the interviews, the participants were briefly explained the purpose and the ethical procedure of the research. As they understood the research process and agreed to participate, they were required to sign the consent form before proceeding to the interview. Interviews were digitally recorded, and notes were taken during the interviews.

During the interview, an interview guideline was used (see Appendix E), which detailed participants' motivation in CouchSurfing and their interaction with guests. At the end of the interview, participants were asked if there was anything to be added concerning their experience in CouchSurfing. Interviews lasted between 30 and 60 minutes each and were conducted between Augusts September 2018.

\subsubsection{Focus Group Interview}

During the process of contacting participants, the researcher was approached by one of CouchSurfing's members to join the local weekly gathering. The meeting serves as a forum for local CouchSurfing members and those who happen to be at the city to meet up and get to know each other. Being 
encouraged to meet other members and collect their experience on CouchSurfing, a focus group interview was conducted.

Building upon the social capital theory (see Chapter 2.2.2), the focus group interview allowed the researcher to investigate the affecting nature of CouchSurfing as a network to its members' behaviour in developing the habit of cooperation, especially in providing free accommodation. The interview guideline was used during the focus group interview (see Appendix F). The questions were open-ended, which was formulated based on the social capital theory to explore structural, cognitive, relational capital, and individual motivation in CouchSurfing non-monetary accommodation sharing behaviour. Regarding this, the participants were initially asked their motivations to join CouchSurfing and the importance of membership to them. While exploring individual motivation, this question also examined how they developed relationships with other members (relational capital) and how their duration of membership (cognitive capital) impacted on their decision-making. The following questions were on structural capital. The participants were asked about CouchSurfing community and its activities in order to examine how participants' behaviours related to regular contact or connection with other members. The last question was regarding participants' roles in the CouchSurfing community. In this section, participants were asked to narrate their involvement in the community. This question explored the possibility of participants' roles in the community raises the responsibility to help others.

Conducted after the gathering, the focus group interview lasted approximately 40 minutes. It involved 6 participants, who voluntarily participated in the interview. The interview was digitally recorded, and handwritten notes were taken. Following the Human Ethics Committee, the participants were informed about the purpose of the research before the interview. As they understood and agreed to participate, they were required to sign a consent form to proceed to the interview. 


\subsection{Data Analysis}

As the process of the data collection was completed, the following research process was analysing the data. The data set was imported, organized and analysed by NVivo software. Following a qualitative thematic analytical approach (Braun \& Clarke, 2006), a comprehensive multi-step coding strategy was adopted, which included: (1) data familiarisation; (2) generating initial code; (3) grouping the codes into potential sub-codes; (4) reviewing the sub-codes; (5) defining and naming the sub-codes, and (6) producing the report. Concerning the analytical approach, the following sub-chapter will now discuss in detail the technique used to analyse the data. Following these multiple steps of coding, sorting, merging and forming final themes (Johnson \& Neuhofer, 2017) an analytical framework will be presented to explain and guide the presentation of the findings.

\subsubsection{Analysis Technique}

The thematic analysis approach used in this research was underpinned by the social constructionism paradigm (see Chapter 3.2), which examined the social processes and interaction behind certain phenomena (Braun \& Clarke, 2006; Burr, 2015). The analysis aimed to go beyond the literal meaning and analyse the assumptions, and systems of meaning underpinning the participants' overt sense making (Braun \& Clarke, 2006).

Therefore, the first step in analysing data was getting familiar with the data. The collected data was restored into NVivo software to be fully transcribed, and every transcript was read in its entirety to build an in-depth understanding of all aspects of data. Subsequently, the following step was generating initial codes. In this step, the data was sorted and categorized into four groups; generated from the conceptual framework (see Figure 2.3), namely motivation, value co-creation practice, resources, and value outcome. The third step was grouping the data in every four preliminary groups into potential sub-codes. They were organised into a broader pattern of meaning (Braun \& Clarke, 2006), followed by reviewing these sub-codes as the fourth step. In this stage, the developed sub-codes were re-examined to check they worked for the entire data 
set (Braun \& Clarke, 2006). Here, pattern and relationship between codes and sub-codes were identified.

The following step was defining and naming the codes, meaning the substances of what every four big codes are about and aspects of the data captured are identified. At this level, the data within the codes was analysed by providing an accompanying narrative for identifying what was interesting about the data and considering how the codes fit into the overall story related to the research questions. During this stage, an analytical framework as a guide to present the findings was developed and will be presented in the following subchapters.

\subsubsection{Analytical Framework}

The multi-steps of coding, sorting and forming the final themes presented in the previous sub-chapter provides an overview of how the data was approached and analysed. Based on this, the following analytical framework was developed.

The analytical framework is generated as a guide to structure the analysis of the data and communicate the findings (D. G. Pearce, 2012). As presented in Figure 3.1, the analytical framework was developed following the conceptual framework and presents the findings sequentially according to the research questions. Starting with a discussion on motivation, part 1 of the analytical framework concerned the reasons for joining CouchSurfing and the decision to share free accommodation. According to the literature, the hosts' intentions for sharing determine the practice of social interaction and explain how values are co-created (Johnson \& Neuhofer, 2017). Therefore, it is important to look closely at the intention of the host for sharing, which is followed by guest preference as the host's judgment of reward and cost before entering the social interaction.

Part 2 concerns the practice of value co-creation between host and guest. As the literature on value co-creation postulates, value emerges as a collaborative effort between host and guest (Prahalad \& Ramaswamy, 2004), meaning that value depends on the interactions between host and guest. Hence, knowing hosts' motivations and how they structure the rewards and costs, and 
social interactions between host and guest will be described. These include the purpose of making interaction, types of interaction that construct the relationship between host and guest, and how motivations influence the interaction.

Part 3 presents finding on resources, components that influence the nature of social interaction (Campos et al., 2015). It is postulated that these resources belong to the domain of the hosts to be applied in making interaction (Campos et al., 2015; S. Vargo \& Lusch, 2004). The success of hosts to generate value from social interactions is affected by these resources. Therefore, the discussions in this part include whether resources belong to the social or physical category. Further, how do the resources affect the interaction between host and guest? Also, how do hosts utilize these resources in conducting social interaction?

The last part of the analytical framework is value outcome. In this part, the findings from part 1 to part 3 are drawn together in order to comprehend the experience of the hosts thoroughly. Therefore, the perception of hosts toward social interaction is explored and compared to the prior motivation before entering the social interaction. 
Figure 3.1 Analytical Framework for Value Co-creation in CouchSurfing

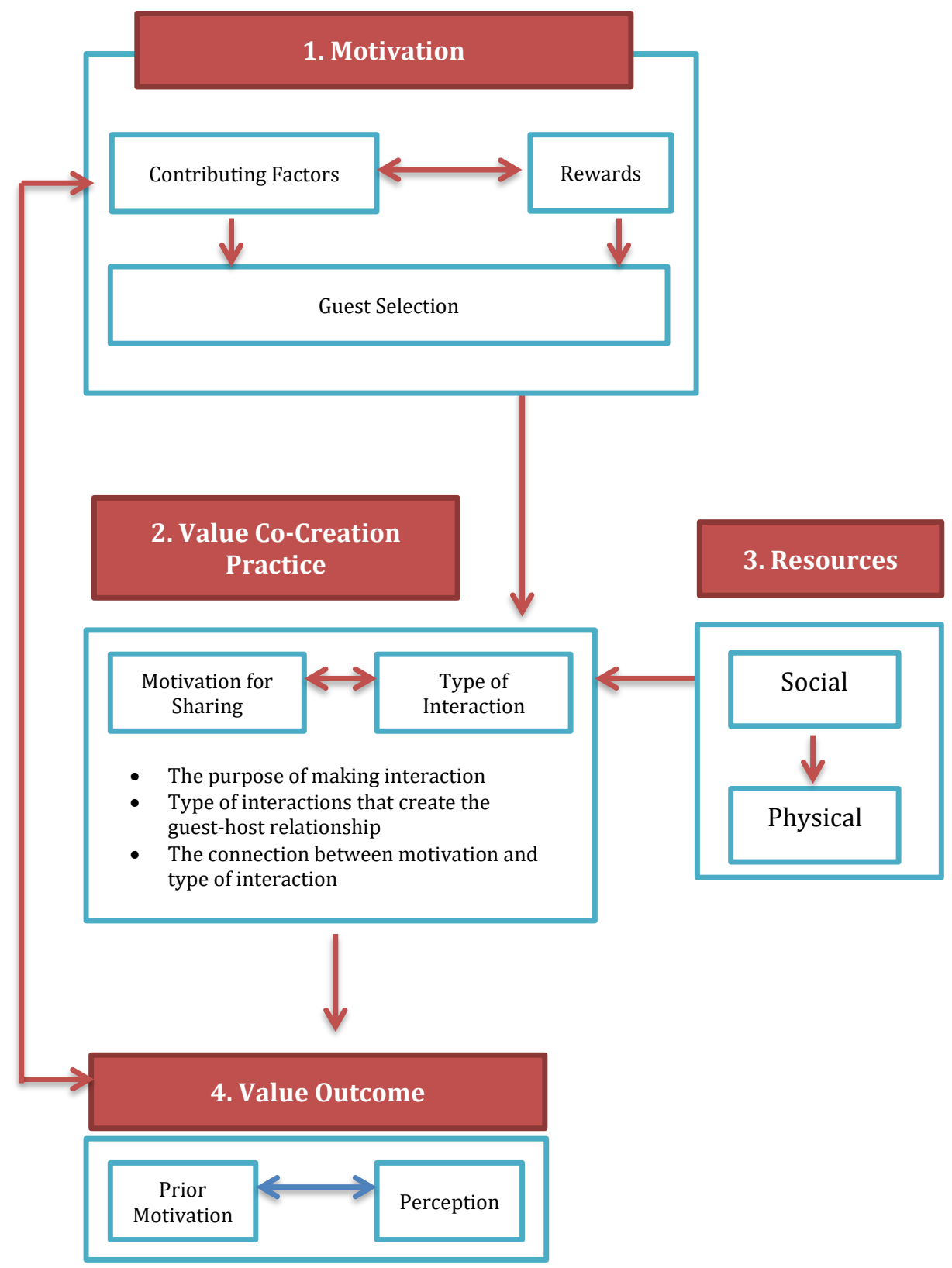




\subsection{Researcher Position in the Research}

The social constructivism paradigm adopted in this research regards objectivity as an impossibility (Burr, 2015; Creswell, 2013). This paradigm perceives researchers are involved in the processes of constructing social phenomena (Creswell, 2013). Therefore, researchers must acknowledge that their background shapes the interpretation (Creswell, 2013), and posit themselves in the research to admit how their interpretation flows from their personal, cultural, and historical experiences (Burr, 2015; Creswell, 2013).

As a researcher, I was born and grown up in Indonesia, which allows me the opportunity to know the different characteristics of people in cities and rural places in Indonesia. Thus, I understand that different regions may have different culture and characteristics of people, which may need a different approach to get along with them. However, my professional career in the Ministry of Tourism equips me with knowledge and the flexibility to approach people from different culture. Therefore, being knowledgeable about the destination and culture was helpful for me to understand the stories the participants shared and comprehend the message they conveyed.

However, being a native influence the way I analyse and interpret the data. Although I tried to be considerate toward participants' perspectives and maintain an unbiased position by distancing myself from the participants, I realize that I understand their stories from my own experiences. Therefore, my position as a researcher provides this research with both strength and limitation, which will be explored in the following section.

\subsection{Research Limitation and Strength}

One of the challenges in conducting the study was to provide an equal number of participants from each region to provide a more objective and comprehensive data. However, the approaching technique did not provide the flexibility to choose participants based on the location. Because $50 \%$ of the respondents were from Bali and the rest from Yogyakarta and Bandung, the collected data mostly reflects hosting experience in the most touristic destination in Indonesia. Thus, it is questionable if the data is also representative of hosting experience in other places in Indonesia. 
Meanwhile, the research method used in the research shows strength in the chosen research paradigm. Social constructivism as the research paradigm allows the researcher to examine the complexity of phenomena by examining the objects behind them and how their characters are generated $\mathrm{Au}, 1998$; Burr, 2015). Thus, social constructivism will lead the research into a comprehensive examination of the motivation and the practice of value co-creation. Further, qualitative research and semi-structured interview adopted in the methodology provides the research with an approach to collect information from the participants in an in-depth manner. Conducting face-to-face interviews and the position of the researcher as a native enabled the researcher to build rapport with the participants, gaining trust to obtain an authentic narrative. 


\section{Findings}

\subsection{Introduction}

The previous section on methodology outlined a set of actions to be taken to investigate the research problems, starting from selecting the appropriate research paradigm, then determining the procedures for data collection and the analytical techniques to identify the information gathered during data collection. The whole process of methodology led to the establishment of the analytical framework in Figure 3.1, which functions as a structure for presenting the findings. This chapter will elaborate on those findings, based on the information gathered in data collection.

The analytical framework presents the data in four different parts, which address each research question presented in a logical sequence following the process of value co-creation. Therefore, the findings discussion will start from host motivation as the basis for value co-creation practice, followed by value cocreation practice, then resource, and finally value outcome. However, before entering the details of the findings, chapter 4.2 will firstly present the characteristics of the respondents, including gender, location, and accommodation type. In addition to that, sample characteristics may also provide insight into how value co-creation practice occurs in the setting.

\subsection{Sample Characteristics}

A total of 20 respondents for individual interviews and six participants for a focus group joined interviews conducted between $10^{\text {th }}$ August and $9^{\text {th }}$ September 2018. As previously explained, a simple random sampling was used in contacting the participants. They were selected based on their location, hosting experience, reference, and active duration of membership. However, according to the ethical research code, the identity of the participants will remain confidential. Therefore, in presenting the sample characteristics, there is nothing, which allows the respondents to be identified. Instead, the respondents will be given pseudonyms, and only some of their characteristics like gender, location and type of accommodation will be presented (see Table 4.1). 
The selection of the participants focused on Java and Bali Island as the most touristic areas in Indonesia. Using CouchSurfing host filtering, participants who resided in Java and Bali were contacted through the CouchSurfing message feature. As a result, on Java Island, participants who agreed to participate were those from big cities such as Jakarta, Yogyakarta, and Bandung. Meanwhile, in Bali, the respondents were scattered in many touristic regions such as Denpasar, Seminyak, Kuta, and Ubud. Since regions in Bali are small compared to those on Java Island, and in order to prevent the respondents being identified, the specific location of participants from Bali will not be revealed. However, during the process of data collection, participants from Jakarta were not confirming their availability for interview, which left participants only from Bandung, Yogyakarta, and Bali. For the focus group interview, it was conducted only in Yogyakarta, which involved six local CouchSurfing members as the participants.

Table 4.1 shows that for the individual interviews, the respondents were equal in numbers between male and female, which limited gender bias in the findings. However, according to the location-based, $50 \%$ of the respondents came from Bali while only 8 participants came from Yogyakarta and the rest were from Bandung. This means that although participants' location was not the most critical factor in undertaking this research, the findings do mostly reflect the most touristic places in Indonesia.

\section{Table 4.1 $\quad$ List of Participants}

\begin{tabular}{|c|c|c|c|}
\hline No & Name & Gender & Location \\
\hline $\mathbf{1}$ & Cinta & F & Yogyakarta \\
\hline $\mathbf{2}$ & Malik & M & Bali \\
\hline $\mathbf{3}$ & Siwi & F & Yogyakarta \\
\hline $\mathbf{4}$ & Karyadi & M & Bali \\
\hline $\mathbf{5}$ & Lily & F & Bali \\
\hline $\mathbf{6}$ & Leo & M & Yogyakarta \\
\hline $\mathbf{7}$ & Wahyu & M & Bali \\
\hline $\mathbf{8}$ & Lia & F & Bali \\
\hline $\mathbf{9}$ & Nadia & F & Bali- Yogyakarta \\
\hline
\end{tabular}




\begin{tabular}{|c|c|c|c|}
\hline $\mathbf{1 0}$ & Agus & M & Yogyakarta \\
\hline $\mathbf{1 1}$ & Ricky & M & Bali \\
\hline $\mathbf{1 2}$ & Miko & M & Bali \\
\hline $\mathbf{1 3}$ & Avelina & F & Bali \\
\hline $\mathbf{1 4}$ & Priyo & M & Bandung \\
\hline $\mathbf{1 5}$ & Umar & M & Bandung \\
\hline $\mathbf{1 6}$ & Arie & F & Yogyakarta \\
\hline $\mathbf{1 7}$ & Angga & F & Yogyakarta \\
\hline $\mathbf{1 8}$ & Maura & F & Bali \\
\hline $\mathbf{1 9}$ & Rio & M & Yogyakarta \\
\hline $\mathbf{2 0}$ & Insan & M & \\
\hline
\end{tabular}

\subsection{The Motivation for Non-Monetary Accommodation Sharing}

'Motivation for non-monetary accommodation sharing' addresses the first research question, which concerns the factors contributing to the decision of hosting in CouchSurfing. In detail, this research question examines which particular rewards were expected by the participants and if the decision to host was related to the obligation to experience and CouchSurfing as a network.

Referring to the literature, motivation is primarily the driving factors behind individuals' behaviour (Fodness, 1994). Thus, looking into individuals' motivation provides the possibility to predict their action. In the context of CouchSurfing, examining individuals' motivation is a basis to understand their behaviour during the encounter with their guest/s and how social interaction between them may occur. Concerning that, knowing individuals' motivation to host will be useful to scrutinise how value can be derived from hosting activity. Consequently, findings on motivation will provide an essential substance for a thorough understanding of hosting experience with CouchSurfing.

Findings on motivation reveal several reasons to provide non-monetary accommodation sharing, which divided into three categories: emotional needs, knowledge, and gifts. Emotional needs relate to pleasure or satisfaction, which centres on basic feelings such as love and empathy. Two motivations are identified under this category: rapport and altruism. Rapport is assuming as the 
need to build a personal connection with others, while altruism is behaviour that shows concerns towards other people.

The second category is knowledge, which relates to the needs of individuals to satisfy their cognitive ability. This motivation encourages individuals to conduct social interaction with the guest to fulfil the need of obtaining knowledge or improving skill. Lastly, the interviews identified gifts from the guest/s as motivation. Having this motivation, participants expected an immediate or tangible reward in return for their accommodation sharing. However, the findings reveal that before entering the social interaction, participants will select their guest based on the criteria they have. It turns out that guest selection appears as a subjective evaluation to maximise the expected value.

Figure 4.1 Motivation in CouchSurfing

Motivation in CouchSurfing:

- Emotional needs

○ Rapport

○ Altruism

- Enjoyment of helping others

- Return the favor

- Indirect Return

- Promoting local culture

- Knowledge

- $\quad$ Gifts 


\subsubsection{Emotional Needs}

Emotional needs relate to individuals' pleasure or satisfaction. Findings reveal that being a host in CouchSurfing provides the opportunity for individuals to fulfil their emotional needs through building rapport with the guest and behave altruistically (altruism).

\subsubsection{Rapport}

Rapport is assumed to be a friendly relationship with other people. During the interview, participants often reveal 'friendship', 'networking' and 'meeting new people' when they were asked about their hosting motivation. These findings indicate participants' eagerness to connect with other people and build a connection with them.

For Insan who had just moved to a new city, hosting through CouchSurfing offered him the opportunity to meet new people and build a relationship. Through CouchSurfing, he got the chance to let or invite someone to stay in his house and build a relationship during the stay. Thus, CouchSurfing connects one individual with another and fulfils their emotional need of making a friend:

well, I live together with a friend in the house. Both of us have this travelling website (Couchsurfing), and we felt that the house was often quiet. Both of us have different activities, whether he went somewhere, and I stayed or vice versa. In that case, the house seemed empty, and I did not know whom I was going to look for, as I was new in the city. I have just moved to the city, and then finally, there was CouchSurfing. (Insan)

For participants who live apart from their family, having guests around them prompted the feeling of being surrounded by family. Guests' presence fulfilled the role of the family, in which participant could have an intimate conversation with and do activities together at home: 
also, it was fun there was someone at home. I live alone in this city, while my parents are in Jakarta (another city). We were spending time together, having many conversations. They were staying for four days, but I was happy because I had got friends to chat, they cooked for me every day. (Nadia)

Meanwhile, for Miko, meeting new people meant distracting him from the negative thoughts he had during his downtime. Companionship provided him with the opportunity to change his perspective through encouragement and support from people he spent time with. Therefore, he joined CouchSurfing as it offered him these opportunities by connecting him with other members:

I just happened to break up with my fiancée at that time ... When I moved to this city, I felt so lonely and so heartbroken. My family suggested me to join CouchSurfing and meet many people from there, distract me from having those negative thoughts, I am not that lonely. (Miko)

Similar to Miko, for Lily, meeting new people allowed her to find encouragement and support from people as she could frankly discussing her personal life without being judged.

one of the reasons why I host in CouchSurfing is that it is easier to talk about personal life with a stranger because they do not judge, they do not know you or the people that we talked about. (Lily)

However, building rapport is not always intended to provoke immediate companionship. Meeting new people from different countries aroused participants' expectation that their guest will return their deed by hosting them or at least give them company when participants visit the guest's country. In this case, building rapport with guest also provides the participants with a sense of security by the expectation that they will not be alone when they are in a foreign country: 
at least when I have friends from different country. Just in case I go to the country someday in the future, at least I have a friend there. However, I do not expect them to return what I have given to them by living in their house, no. (Nadia)

\subsubsection{Altruism}

Altruism can be understood as the behaviour of seeking to benefit other people even at absolute cost (B. F. Meeker, 1971). Providing non-monetary accommodation sharing, participants feel a sense of achievement as they accomplish things of value. They found their deed as something valuable and from there they gain happiness from helping others. The findings reveal that altruism in this study manifest in four different forms, namely enjoyment of helping others, returning the favour, indirect return, and promoting local culture.

In the first identified altruistic motivation, enjoyment of helping others, participants found contentment by providing non-monetary accommodation sharing. They perceived that being able to help others was a self-achievement:

I am not looking for anything (by offering free accommodation sharing). If I can help other people, I feel happy, and content. (Ricky)

For some participants with this motivation, they also perceived that guests with CouchSurfing are travellers on a budget. Therefore, they believe that helping guest in CouchSurfing is an urgent matter and they do not need reasons to help someone in need:

in the case of CouchSurfing, I think people should offer help. If someone asks for help, help, we do not need to consider who is this person asking for help and whether our deeds will be paid or be exchanged into something else. Those sending requests to stay for free are those in needs, maybe they need the help of settling in a new destination. You know, travel is costly. (Agus) 
The second identified form of altruism is returning the favour the participants had received when they travelled as guests through CouchSurfing. Having received friendly treatment without any compensation from the host, who is entirely a stranger, leaves participants with gratitude and indebted. Therefore, hosting in CouchSurfing offers them the opportunity to return the kindness they received by doing the same thing to their guest:

when I returned to Indonesia, I felt like I wanted to give it (the experience) back and I thought it was pleasant when someone who is entirely stranger hosted you without expecting anything. (Nadia)

However, although the definition of altruism indicates that participants do not expect something in return, it does not mean that there is no expectation at all. Some participants believe in karma, and they believe that their good deed of helping people with CouchSurfing will be returned to them indirectly, either to them or to someone close to them:

I believe our good deed will return. I never expect anything though. However, maybe someday when my kids go abroad there will be someone who helps my children, especially in urgency matter ... It may not directly be returning to me, but maybe to my children or my grandchildren... (Avelina)

The reason for doing it [hosting] is because I am paying forward. I am doing this with the hope if someone I love, or I care needs help, there will be someone to help him or her. I believe in Karma. (Miko)

The last form of altruistic motivation identified in the CouchSurfing context is promoting local culture as part of participants' concern for the culture. Through hosting and meeting people from different background, participants see an opportunity to introduce and promote their local culture. Thus, they perceive that hosting is part of their duty towards their culture: 
if you are interested [with my profile], please come. I will introduce you to Javanese culture: gamelan [music instrument], dance, and beliefs. I can offer you to live like a Javanese. (Agus)

Still regarding promoting local culture, hosting in CouchSurfing allows Karyadi to share his perspective. He recognised that Bali in the past offered a better intimacy than nowadays. Encouraged by the desire to make Bali friendlier and more welcoming, he decided to join CouchSurfing and show people he met what the true Bali looked like, according to his viewpoint:

on the other hands, I perceive that Bali is not Bali as what people used to remember as friendly Bali. People no longer show intimacy. Bali is leading to more commercial right now. I want Bali as what people captured, friendly and welcoming. (Karyadi)

\subsubsection{Knowledge}

Hosting in CouchSurfing attracts participants in the way they can exchange knowledge with the guest. As became evident in interview, participants explicitly did not require any monetary exchange by offering people accommodation sharing in CouchSurfing. However, they expected their guest could give knowledge as something in return. In other words, participants want the guest to share their resource:

[I expect] knowledge ... I expect they [guests] have something I can learn, something that they can share. Just consider it as a way to pay the services [accommodation, information, and so on] I have given to them. I know they are all free monetarily, but they can pay the services through the knowledge they have. (Agus)

The findings also reveal that CouchSurfing facilitates participants to gain knowledge by allowing them to experience a different culture. Living with their guest, the participant has the opportunity to interact with guests, exchange ideas 
or observe a guest's habit to learn about their culture:

I am more into the cultural exchange. I like to see the behaviour of people from other countries or tribes in Indonesia, their daily life. These are interesting, and that is why I am interested in culture and people from CouchSurfing. (Leo)

In addition to cultural exchange, CouchSurfing allows participants to expand their travel knowledge by sharing ideas and stories with their guest. Sharing travel experience provides the participant with information needed for their hobbies and also factual knowledge on a specific destination:

I am searching for some travelling experience. I like travelling so much, so that is why I want my guest to share (their experience) with me. (Insan)

However, for the participant with financial constraints to travel, exchanging travel experience is the same thing as travelling. Sharing stories and experiences, participants gain information on touristic destination other than Indonesia. This, it increases participants' horizons and knowledge about a specific destination:

I am a gregarious person. I love to chat, hang out. The thing is I cannot afford travelling by my expenses. I do not say it is impossible, but it is so hard to achieve. So, I am travelling by making friends, through their story they share. (Wahyu)

For participants whose interest is learning a language, CouchSurfing is a useful place to improve their language skills. Through CouchSurfing, participants can meet people who speak different languages. In that way, the participant can communicate in the language they wish to improve with the participants:

at first, I just wanted to make friends, but then I wanted something else, my English was poor. So, I thought I was going to improve my English through 
them [the guest]. Why not? They stay for free, but I can have an English conversation with them. Then I got a friend as well. (Wahyu)

Lastly, the findings also show that in less touristic places such as Bandung (compared to Bali), the interaction between foreigners and local people is rare. There is a gap between foreigners and local people, which create a negative stigma towards foreigners. By hosting, Arie wanted to minimise the gap between local people, at least within her family, by facilitating knowledge sharing during the stay:

because of the lack of knowledge itself, Indonesians have a certain stigma about foreigners. Hanging out with strangers can erode that stigma. It also makes us more open-minded and open to new things. (Arie)

\subsubsection{Gifts}

Obtaining a gift was not a dominant motivation showed by the involved participants. Two participants acknowledged their motivation to join CouchSurfing and provide non-monetary accommodation sharing as gifts. Further, they mention that they explicitly stated this motivation in their profile and conveyed directly to their guest during their correspondence. They believe that bringing gifts to someone's house is part of Indonesian culture and guests should know the culture:

at first, it did not mention about the gift, but for sometimes, I think they have to know our culture. Then, I wrote in the profile to bring something if you come and visit someone's house in Indonesia. (Angga)

Apart from being considered, as part of the culture, for Leo, a gift is a resource of exchange between him and guest. He perceived gifts as something in return for his deed in providing accommodation sharing: 
there is no money attached, but there is a currency that you [guest] have to pay, that is kindness. If you come to somebody's house, you do not come empty-handed. You should bring something, especially if that is Indonesian/ Asian culture ... I say nothing is free, and there is another currency you have to pay, that is kindness. (Leo)

While the gift is not a dominant motivation in CouchSurfing context, it is interesting to note that this motivation is not purely based on the needs of tangible rewards. Instead, findings show that aside from the need for a gift, there is an altruistic motivation underlying the decision to host, in which participant feels the sense of obligation to introduce the guest to their culture.

\subsubsection{Guest Selection}

The previous chapter on motivation has described the motivations developed by participants in CouchSurfing. It has been previously mentioned at the beginning of the chapter that having the motivations, participants develop an effort to maximize the outcome by selecting their guest. Following that, this chapter will now present thus the process of selecting guest, in which participants set some criteria to help them decide which guest they are going to accept.

Most participants select their guest according to their subjective consideration of reward and cost. On that account, participants will accept a guest, who provides them with benefits. The findings reveal that guest profile and length of stay are essential to help participants in the decision to accept a request. Through a guest profile, participants can decide their compatibility with the characteristics of the person with whom they are going to stay. While in the length of stay, participants consider if the guest's duration of staying provides them enough time to achieve their mission.

Before proceeding to discuss the criteria of guest selection in detail, it is essential to first look into how these criteria link to participants' motivation. Table 4.2 shows that participants with specific motivations will set different criteria than those with other motivations. Participants with rapport, knowledge, 
and business motivation are looking for a guest who wants to build a relationship. For rapport aspiration, having common ground with the guest is another point to note in selecting the guest. For a host with knowledge aspirations, having someone knowledgeable is a must on the list. However, both rapport and knowledge aspirational hosts agree that length of stay is essential in considering the decision.

Meanwhile, it is difficult to identify the criteria set up by altruistic aspiration, as most participants with altruism do not show their aspiration as a single motivation in their acts, only Agus and Ricky. Having no other motivations, Agus and Ricky will accept all requests from CouchSurfing without any guest filters. The same thing goes for gifts as motivation, in which participants with this motivation also showed another motivation. Therefore, it is difficult to identify for participants with gift motivation, which filter they use in selecting their guest.

Table 4.2 Guest Selection by Motivations

\begin{tabular}{|l|ll|}
\hline \multicolumn{1}{|c|}{ Motivation } & \multicolumn{1}{c|}{ Guest Profile } \\
\hline Rapport & $\bullet$ & Build a relationship (making \\
& & interaction) \\
& $\bullet$ & Common ground \\
& $\bullet$ & Length of stay \\
\hline Altruism & - & \\
\hline Knowledge & $\bullet$ & Knowledgeable \\
& $\bullet$ & Build a relationship \\
& $\bullet$ & Length of stay \\
\hline Gifts & - & \\
\hline
\end{tabular}

\subsubsection{Guests'Profile}

It is assumed that certain motivations will lead participants into considering different criteria for guest in their selection. They will choose guests based on what they are looking for in CouchSurfing. The findings show that in 
selecting guests, most of the participants will look into guest's profile and see if what is written in the profile is able to benefit them in certain ways.

On the CouchSurfing platform, a member's profile will contain detailed information about the member, including gender, age, character (what do like and dislike), reason for joining CouchSurfing, what member can offer as guest/host (in teach, learn, and share section), references from previous contacts both from host and guest, and photos the member has taken during travelling. Exploring the profile, participants investigate to what extent the owner of the profile will be compatible with them, and supportive in the accomplishment of their mission before they make the decision. Alternatively stated, the profile picture is essential in CouchSurfing hosting decision-making. To show its importance, Leo will not wait for an interesting profile to request to stay. Instead, he will first invite the person and offer the person his house to stay:

[in case I see] an interesting profile, I will approach the member. I will take the initiative to offer the person to stay at my house, instead of waiting for strangers to request. (Leo)

Despite that, participants with rapport and knowledge as motivation tend to choose sociable guests, those who want to build a relationship with participants. In searching for this type of guest, participants will subjectively make a judgment from the profile or by having correspondence several times with the guest candidate before they decide. Mostly, these participants are concerned with there being something in common between guest candidates and themselves. Those who possess common ground with participants manage to have more significant opportunities to be accepted as guests in participants' house:

before they arrived, they had to send an email, and I had to have correspondences several times even though only via email. [I must know] what they look like, their characters, and so on. If there was not something in common between us, it was better to decline than having boring conversations during the stay. (Rio) 
Malik, while looking for something in common, also uses photo galleries of his candidate. For him, photos are useful to identify if the candidate has the willingness to interact with him during the stay. Through the photos, Participant tries to indicate the candidate's behaviour and travel style:

mainly I see what they [the guest] wrote. Their profile, is there anything mutual with me? ... The second one is their photos because it is said that photos can say more than what people write. ... If they took a photo with local people, it would be more interesting for me because I may conclude that they travel not to take photos in mainstream places, but because they want to connect with local people. (Malik)

Lily, meanwhile, preferred candidates who were sociable people and was eager to make the interaction between them through correspondences they have. During the correspondence, Lily would see if the candidate is enthusiastic in meeting her or just in things about the candidate or that the candidate cares about. Based on that evaluation, she will favour the candidate with enthusiasm to meet her as host:

I will usually have correspondence with the candidate first [through CouchSurfing chatting feature]. I need to know first, what is it he or she talks about? Is the candidate excited to meet me? Alternatively, is the candidate excited about him or herself only? I can tell from there. (Lily)

Another thing to consider from the candidate's profile is the reference from his or her previous contact, both from a host and from a guest. An opinion written by someone else about the candidate provides insight into the characters of the candidate in CouchSurfing situation:

I will read the references, both the positive and the negative ones ... I did not select the guest before. However, now I am into those with few references. Those with many references tend to be acting up; they play drama. 
However, a newbie in CouchSurfing, their expressions seem to be sincerer. (Rio)

For Maura, reference and recommendation from a mutual friend will guarantee the candidate's behaviour. For this reason, the candidate will have significant opportunity to be accepted as a guest:

I am more into surfers who were recommended by a friend. Their chance to accepted as my host was bigger. However, still, I will ask them to request formally from CouchSurfing platform, because it is an appreciation for people who give the accommodation. (Maura)

However, based on the host perspective, the way candidates approached participants through their request could also predict their behaviour. Guests who wrote less personal requests and were not communicative in conveying their message had little opportunity to be accepted. For Miko, this kind of guest is a 'freeloader', who only needs a place to stay but would give minimal effort to be in contact with the participant as host.

something like, "Hi, my name Puja. I want to stay in your place, but your place must have fast Wi-Fi, hot water, and so on ... I am not going to do anything with that request ... They only need the accommodation. Geez! Big no! (Miko)

For a participant with knowledge aspiration, they do not only require a sociable guest but also someone with knowledge, so they can exchange ideas or learn from guest experience.

I am a quite selective host. I only host those whom I think are impressive ... Let us say he had experience about something, let us say he works in an NGO, volunteers of a natural disaster. Is he a bio-marine? I want to know the world of others, and from them, I can learn. (Leo) 
For Priyo who is interested in improving his skill in foreign language, he prefers guest from a specific country, which speaks a particular language he has interest to learn. Therefore, he tends to choose a foreigner instead of a local guest, as his motivation requires him to choose someone with specific resources:

I am not into locals; I need to gain experience with foreigners, learning from their way of thinking and their habit. With them, I have a new experience, networking and my (improve) language skills, especially the language I am currently learning: Japan, German, France and Mandarin. (Priyo)

Two respondents acknowledge that they select their guest based on age and gender. This preference for knowledge is based on his previous experience on CouchSurfing. For Rio, he perceives that younger surfers tend to be misbehaved an immature. Therefore, he prefers someone above 30 years old:

I am not into younger people as most of them are misbehaved and inconsiderate. I am more into mature ones (above the 30s). They tend to have better awareness and [be] polite as guests. (Rio)

In addition to that, although minor, gender also takes place in the decision. The participants with this preference perceive that male guests tend to be more straightforward in their attitude. Therefore, they are easier to manage and to have as a guest.

I prefer to host male guests as they are more straightforward ... they are more independent. (Lia)

\subsubsection{Length of Stay}

While participants look into guest's profile to investigate their characters and compatibility, the findings also show that guest's length of stay is essential in determining participants' decision to accept a request. Through the length of stay, the participant will consider if the duration of stay allocates them enough 
time to accomplish their mission. Concerning that, findings show that participants with rapport and knowledge aspirations prefer guests staying for longer durations. Therefore, these participants will not accept guest who requests to have an overnight stay as they perceive that it is impossible for them to have interaction with guest and get to know them:

if only staying for one day, it is not CouchSurfing. They are just looking for free places. Why do not they go to a hostel? Because what I know in CouchSurfing, you have to share, you must have interaction with your host. By staying overnight, what interactions can you get? (Maura)

\subsubsection{Conclusion Summary}

Discussion on motivation serves as a fundamental substance for value cocreation practice, in which participants' behaviour can be predicted, and value as mutual resource exchange can be identified. The findings have elaborated the motivations to provide non-monetary accommodation sharing through CouchSurfing into three major categories, namely: emotional needs, information, and gifts. Presentation on findings in these three categories shows which values participants expect in CouchSurfing.

Under emotional needs, participants showed rapport and altruism as values they were after. CouchSurfing allows participants to gain new friends, encouragement and support, something to expect in the future from their guest, and also happiness by helping others. Meanwhile, in the knowledge category, CouchSurfing provides participants with the opportunities to experience a different culture, exchange travel experience, improve language, or even change the perspective of locals to foreigners. The last category is the gift, in which participants expect a present from their guest in exchange to their favour. While this motivation shows tangible reward, this motivation is not purely driven by the need of the participant to extrinsic value. Instead, in this motivation, the need to do something concerning the local culture is also well reflected.

Having the motivation, participants' show that there is a subjective judgment to be made before they enter social interaction, through selecting their 
guest. Each motivation shows different criteria of guests as they show different needs in value. However, for altruism and gift motivation, it is quite difficult to tell which criteria for guests they prioritise because participants with these motivations usually possess another motivation in addition. Therefore, the findings show that a purely altruistic participant tends to accept all guests without any filter needed. Chapter 4.4 will now look closely on value co-creation practice, in which social practice between participants and guests will be discussed in detail. This chapter will identify which interactions occur between host and guest in the CouchSurfing context and how motivations, which have been established in this chapter influences those interactions. Further, discussion on value co-creation practice will include the role of hosts in the interactions, which will provide insight into how they derive and determine values from social practices.

\subsection{Value Co-Creation Practice}

The previous chapter has elaborated different motivations showed by participants to host in CouchSurfing and what rewards/values are expected in each motivation. The finding also shows criteria of guest set by each motivation as a method to maximise the value. As motivations to drive participants to host in CouchSurfing have been identified, the findings on value co-creation practice will now be discussed.

To begin with, it is essential to refer back to the definition of co-creation in tourism studies. Campos et al. (2015) define co-creation as "a process of interrelated interaction and activities that connect the tourist and other actors, and experiences are the context in which those interactions and activities occur" (p. 1311). Based on this definition, it is assumed that experience can only be cocreated in surroundings in which guests and hosts make interactions and influence each other's experience (Campos et al., 2015; Schuckert et al., 2017; S. L. Vargo et al., 2008).

Regarding the research question, interrogation of value co-creation addresses the research question 2, which concerns with social practices between host and guest in the CouchSurfing context, primarily related to how and why 
these social practices occur. As social interaction plays an essential role in determining experience, identifying which interactions occur between actors provides a fundamental part of how values are co-created during the encounter. In order to accommodate these issues, research question 2 will address the following questions: Which interactions occur between host and guest? How does motivation influence interaction? What is the role of the host in the interaction?

Further, findings on value co-creation practice reveal that interaction between host and guest in CouchSurfing, namely building rapport, knowledge sharing, insight into local's lives, and least interaction. These interactions are categorized based on the primary motivation identified. However, it is found that motivations behind these interactions are dynamic. Instead of having a sole motivation in every interaction, findings show that motivations behind these interactions do not rigidly stem from one motivation only. Rapport, knowledge, and altruism are identified as motivation in every category of interaction. However, it is interesting to know that for some altruistic hosts, having least interaction or no interaction at all with the guest during the stay is enough for them to derive value from hosting in CouchSurfing.

Before further discussing findings on value co-creation practice in detail, Table 4.3 explains which interactions occur during the encounters and how they relate to motivation. While the table provides an understanding of how motivation affects social interactions, this correlation between interaction and motivation also serves as basic knowledge, which is useful in analysing value at the end of the value co-creation process.

Table 4.3 Social Interactions in CouchSurfing

\begin{tabular}{|c|c|}
\hline \multicolumn{1}{|c|}{ Interaction } & \multicolumn{1}{|c|}{ Motivation } \\
\hline Building Rapport & $\bullet$ Rapport \\
- Welcoming & $\bullet$ Altruism \\
- Cooking and eating together & $\bullet$ Knowledge \\
- Hangout together & \\
- Sharing emotion & \\
\hline
\end{tabular}




\begin{tabular}{|c|c|}
\hline - Touring with guest & \\
\hline $\begin{array}{l}\text { Knowledge Sharing } \\
\begin{array}{l}\text { - } \\
\text { - } \text { Peep conversation } \\
\text { - } \text { Learning new skills } \\
\text { - } \quad \text { Shared learning }\end{array}\end{array}$ & $\begin{array}{ll}\text { - } & \text { Knowledge } \\
\text { - } & \text { Building rapport } \\
\text { - } & \text { Altruism }\end{array}$ \\
\hline Insight into local life & $\begin{array}{ll}\text { - } & \text { Rapport } \\
\text { - } & \text { Altruism } \\
\text { - Knowledge }\end{array}$ \\
\hline Least Interaction & - Altruism \\
\hline
\end{tabular}

\subsubsection{Building Rapport}

In value co-creation practice, every interaction, which was purposed mainly to build connection and closeness with guest, is categorized as building rapport. Under this category, both host and guest are involved in joint activities, namely welcoming, cooking and eating together, 'hanging out' together, sharing emotion, and touring with the guest.

\subsubsection{Welcoming}

The first identified social practice in CouchSurfing context is welcoming. Welcoming occurs at the beginning of the host/guest relationship, and marks the first physical meeting and greeting from hosts (Camilleri \& Neuhofer, 2017). From a building rapport perspective, welcoming can be seen as a chance to introduce guests to CouchSurfing homes and local environments. However, welcoming can also be considered as a good start for hosts and guests to get to know each other.

For a welcoming, findings reveal that most participants will pick up guest at the airport or set up a meeting point. As for Leo, his picking up his guest is an expression of excitement from host to meet the guest. His previous experience as a guest in CouchSurfing made him perceive that it is necessary for hosts to pick 
up their guests. This gesture gives guests a memorable experience, as they feel welcomed and secured, especially in a new place. Thus, based on Leo's experience, welcoming can be seen as an act of building rapport but also a way to return the favour he has received in his previous experience:

if I do not have something to do (although I expect them to be independent), I will pick them up. From my previous experience as a guest, being picked up by host means something ... It means something, especially for someone who comes to the city for the first time and they do not know what the city looks like. (Leo)

For Rio, his welcoming is part of the altruistic motivation. For him, picking up his guest reduces the risk of guests to be lost on their way. Meanwhile, welcoming provides him with a good start to begin the relationship as they can build communication on the way home:

I prefer picking them up to hearing they lost on their way to my house. It will be easier for me that way. We can straight back home, or we can have a light conversation, talking about their plan here. (Rio)

However, some hosts prefer to meet their guest at home. They will give their guests the address and guide if necessary. While guests arrive at home, hosts will welcome them by showing them around, giving them a house tour and information about what is in there in the local environment:

if they have arrived, I will show them around. I will explain what is in there nearby, asking them what their daily needs are? (Ricky)

Meanwhile, for Lily, welcoming is an altruistic deed. It is a chance to promote local culture. Therefore, when guests have arrived in her accommodation, she will take them to the local food restaurant in order to have a glance of Indonesian culture. 
I will take them to street food/local food restaurant ... I will take them to local Indonesian food because I want them to know the Indonesian culture. (Lily).

\subsubsection{Cooking and Eating Together}

The findings show that half of the participants spent time with their guest by cooking and eating together. For hosts with packed schedules, cooking and eating together was the possible interaction they could do with their guest:

I will take my guest somewhere before going to work. I do not have flexibility in my working hours now. So, I guess, I can have dinner together. (Cinta)

Further, cooking and eating activities provided participants with opportunities to build intimacy and connection with their guest. For Nadia who lived apart from her family, cooking and eating together was a moment to have fun and share an intimate moment like a family, in which she could also share stories and ideas:

sometimes they cook for me. So, it was more on having a moment together, laughing together, sharing stories. It is always fun you know, going back from work and someone is waiting for you to eat together. (Nadia)

\subsubsection{Hangout Together}

Hangout together or spending time with guests for recreational purpose or socialising is another practice of building rapport in CouchSurfing. The findings show that this activity occurs in two different spaces: CouchSurfing homes and the local environment. Within CouchSurfing homes, Arie, who lives with her big family, exemplified that hanging out with the guest at home always turned out to be an intimate moment for her and her family. Especially when her family is never going abroad, spending time with foreigners allows them to have shared learning about different cultures: 
I concluded that my family was happy meeting my guest was when they have the conversation. We have seen foreigners a lot but never have any interaction. So, it is the moment I introduce my guest to my family, and we start talking to each other, introduce our traditional food ... there was also this guest who brought a postcard collection from his country and tell us about those exciting places. Moreover, my family will also tell him places in Indonesia, which is similar. (Arie)

However, outside CouchSurfing home, hosts and guests spend their time together by partying, hanging out in the café or going to movies:

we usually hang out at café, after that we go partying [to a night club]. (Miko)

\subsubsection{Sharing Emotions}

Sharing emotions is an activity, in which both participants and guests share their personal feeling and experience. As part of building rapport, this activity provides both actors with the opportunity to understand each other, gain support, and build closeness:

we have pillow talk ... share stories, personal things. Some guests of mine shared the reason why they travelled. Some were heartbroken. (Cinta)

Meanwhile, sharing emotion is also a way to release emotional burden. While gaining mental support, sharing emotion also relieves emotional stress. In case of Arie, sharing emotion with guests provided her with encouragement and support for her problems:

I also shared them my dilemma that I was nervous about going back home and all kinds. So it is like sharing all the complications in our life. (Arie) 
However, for Lily, she perceived that sharing personal stories with strangers provided her with a more objective and less judgmental opinion. For some participants, it was easier to talk about their personal life with the guests in CouchSurfing than someone they knew. Through her guests, Lily could release her emotional burden without being sentimentally judged:

we talked about family, his bad relationship with his brother but they made up, while I did not. That is one of the concepts why I do CouchSurfing. It is easier to talk about personal life with the stranger because they do not judge; they do not know you or the people that we talked about. (Lily)

\subsubsection{Touring with Guest}

This building rapport activity occurs outside CouchSurfing home, in which participant and guest visit touristic places at the local destination together. Providing a guest with a companion to visit local touristic places allowed hosts to spend time together with their guests and build the relationship. Sometimes, touring with guest also built the participant's knowledge about the local destination:

I have no ideas that Bali has lots of things. Sometimes I knew that from CouchSurfing member I was hosting, and they took me there. Because he could not ride a motorbike, I gave him a ride ... however, I thought to take a road trip with friends was always be a memorable experience. (Malik)

However, the findings found that touring with the guest was also part of altruistic behaviour. Taking part in creating guests' tourism experience provides participants with a sense of satisfaction by making others happy:

I will try to make people happy. If I have free time, I will take them anywhere they like, it is kind of normal for me. (Priyo) 
Further, touring with a guest was also driven by the need to exchange knowledge and practice altruistic behaviour. Giving the guest a companion with whom to explore touristic places allowed hosts to improve their knowledge on the places/culture, share that knowledge, and at the same time promote and preserve the culture:

when you try to introduce your culture to them, I will research this place and introduce them to the culture, history, and so on. When they get knowledge from it, they help me preserve it. (Miko)

Also, touring with guests provided hosts with the opportunity to obtain specific knowledge from their guest. It occurred in case of hosts meeting guests with particular expertise. This way, giving guests touring companion expanded hosts' knowledge through the learning of particular information about the destination, which is little known even by locals:

I did not know that there is a temple called Kendulan in Yogyakarta. If it were not because of my guest, I would not know that ... As my guest was an archaeologist, he knew that kind of stuff. For me, this is a new thing, and I expect things like this. (Nadia)

While for Rio, giving his guest travelling companion is part of his job. He uses the opportunity to capture the guest's impression about the places and use that as a substantial resource for his job:

I am writing a travel blog, which requires me to take pictures and write people's experience in that place, how they perceive the place, and so on. while spending time with them, at the same time I am doing my job too. (Rio) 


\subsubsection{Knowledge Sharing}

Every interaction in a host/guest relationship in CouchSurfing with the primary purpose to obtain knowledge or transfer knowledge is categorized into knowledge sharing. Based on these criteria, activities under knowledge sharing can be identified as deep conversation, providing tips and advice, learning new skills, and shared learning.

\subsubsection{Deep Conversation}

Most hosts with knowledge aspirations will make an effort to obtain knowledge from their guest through in-depth conversation. In a deep conversation, instead of having small talk, both actors prefer to have serious topics for their discussion that may range from politics, history, or religion according to their interest. Mostly, a deep conversation is something unplanned in social interaction. Sometimes, it spontaneously occurs during cooking and eating together, or during the hangout.

As part of knowledge sharing, deep conversation allows hosts to satisfy their curiosity and broaden their knowledge. In addition to that, having a deep conversation provides both hosts and guest with the opportunity to exchange ideas about sensitive issues and obtain a new perspective.

I got many benefits and knowledge (by talking to guest). I am interested in public policy ... I also always ask about the regulation in the country. In several countries, someone can get a tuition allowance and unemployment benefits. I am just amazed that what I think as possible here, impossible anywhere else. (Miko)

I once hosted a woman from Armenia ... When I met her, we were talking about Armenia, so she explained to me that Armenia was near Azerbaijan. She told me about the history ... I studied IR (international relations) with a focus on the Middle East, so the story just captivated me. (Cinta) 
However, the deep conversation also enabled hosts to acquire as much information as possible from their guests. In the case of having guests with specific knowledge, deep conversation provided hosts with the opportunity to explore the knowledge in an in-depth manner:

my sister and I have a target in these 2 or 3 years to go to El Camino de Santiago. Not many people know about El Camino de Santiago, it is a kind of religious pilgrimage ... There was my CouchSurfing guest who has followed the journey, and he could tell me everything in detail. I cannot find it through Google (Nadia).

In-depth conversation also occurred between the hosts' family and guests. Arie exemplifies how the impact of deep conversation on religious issues occurred between her family and her guest. While the deep conversation impacts the way guest perceive the religion, from Arie's perspective, the conversation provided her with fulfilment as it relates to her identity:

when she went home, she talked a lot with my father about Islam. My father was a religion teacher, and they had a serious discussion. She said that she got to know more about Islam by talking to my dad than by spending months even years maybe reading about Islam in a book. (Arie)

\subsubsection{Providing Tips and Advice}

As part of local culture, hosts possess factual knowledge about the destination, which enables them to share tips and advice to a guest. While sharing this knowledge to guests through providing tips and advice, this activity is also part of hosts' altruistic motivation. Avelina provided her guests with recommendations local tourism spots to help them spend their time effectively in the destination: 
I tried to have a conversation with them, and we talked about places they could go to, some natural tourism spots. They were happy because they knew where to go, by so they did not waste time here. (Avelina)

Hosts did not only recommend favourite tourism spots, but also nonmainstream tourism spots. While this may be a valuable proposition for guest, from hosts' perspective, they were helping to promote less favourite tourism spots at the destination:

I do not like taking people to mainstream places. I will recommend my guests places; which people know little like rice fields in Jatiluwih or Klungkung. Bali is not in Southern part only. (Priyo)

Besides an itinerary for exploring the local destination, tips and advice from a host may take form in how to adjust into local life. This social practice relates to hosts' recommendations on local people's trait, local transport, security tips, and price range and bargaining when it comes shopping:

[I can recommend] Trips and travelling in Indonesia, from low to high budget. I can also tell them Indonesian general trait or trait people from a specific region in Indonesia. (Insan)

I will pick them up, and I will let them know the dos and don'ts, which ATM is secure, what kind of scam happen in Bali so they will not get fooled here (Lily).

\subsubsection{Learning New Skills}

In this social practice, participants were involved in the interaction with guests to learn and obtain some skills. In questioning the types of skills learned in this social practice, findings reveal that cooking traditional food and playing a musical instrument were the most common new skills transferred from guests to hosts: 
we cooked traditional food from the Philippines. I forgot what it is called; it is chicken with vinegar. (Cinta)

Some of them gave me musical instruments from their countries; they taught me how to play it. (Agus)

Other than learning traditional food and music instrument, Agus explained that he also learned how to read a geologic map as life skill from his guest. Gaining new skill, it supports his hobby in exploring a new destination:

I learn how to read a geologic map from my Ukrainian guest. He liked climbing a mountain without a guide, by only using the map. It is fascinating to me because I like exploring. Be[ing] able to read the map is very helpful. (Agus)

\subsubsection{Shared Learning}

Shared learning is an activity, in which both hosts and guests work collaboratively in a specific project. This social practice occurs when both hosts and guests have a common ground and are passionate about the similarity. Interviews revealed that through this practice, both actors can learn from each other in order to improve the skills they have:

when they were in my place, we made a collaboration video. It was fun because I used to write music, so it was like a connection ... we all were passionate about music. Many things to share and we worked randomly but it turned out to be so good ... and they also helped my final assignment, documentaries. (Arie)

\subsubsection{Insight into Local Life}

Another common practice of value co-creation in the CouchSurfing context is an insight into local life. This practice encompasses activities, which relate to showing guests local culture and local everyday life. Most participants 
conducting this social practice are encouraged by their altruistic motivation but also the need to build rapport and share knowledge.

During interviews, the participants acknowledged that in conducting this social practice, they did not make much effort in introducing their daily lives to guests. This activity does not as affect their daily routine as guests were involved in their daily routines, such as eating together, getting along the big family and even helping hosts with chores:

celebrating Eid al-Fitr, I included them in the preparation. We went to the market, made a ketupat [rice wrapped in coconut leaves], we ate together, and after that, we went to see Takbir [a parade of celebration]. I mean that what local do, right? ... As I told you, I do not make much effort, just regular, daily activity. I taught them how to make rujak (fruit salad with peanut sauce), we picked fruit, went to the traditional market. (Maura)

He wanted to boil water, and I taught him to do that. He helped me clean up, play football with my nephew; he even wanted to help my mother bought some gas bottles to be sold in our stall. (Angga)

Further, insight into local's life also occurs through talking about Indonesia and its culture, teaching guests how to make local craft and cooking together. By doing so, hosts address their need for rapport, altruism and knowledge:

we talked about Indonesia, its customs and culture and then they asked about religious activities, how people pray. Then, they want to know how to make canang [an offering craft] ... we cooked together at home, sometimes their traditional dishes, sometimes Indonesian dishes. (Avelina)

Apart from introducing culture and daily routine, insight into local's life also takes forms in the way hosts recommend guests places to hang out. While doing so, hosts will take the opportunity to introduce which belongs to local culture and which does not: 
I usually try to introduce guests on how to hang out like a local. For example, alcohol is not our culture. Therefore, I will never take the initiative to take them to the places, whereby alcohol is hugely consumed unless they ask me to take them there. However, I will explain to them first that if they want to know the local's life, we do not hang out there. (Maura)

\subsubsection{Least Interaction}

For some altruistic hosts, hosting in non-monetary accommodation sharing is part of their enjoyment to help others. Hosts who show the need to help others as their dominant motivation in hosting, they do not require any interactions from their guests. Instead, their expectation towards guests is very low and therefore it is enough for them to provide accommodation and information needed. By doing so, they have sensed an achievement, and it also provides them with satisfaction.

\subsubsection{Conclusion Summary}

Findings on value co-creation practices identify interactions between host and guest in CouchSurfing context as building rapport, knowledge sharing, insight into local life, and least interaction. These categories are created based on the primary motivation identified, which encouraged hosts to conduct the interactions.

It can be assumed that motivation performs a significant function in shaping these interactions. Findings show that the motivation behind these social interactions is dynamic and does not rigidly stem from one motivation only. This means that in conducting these social practices, a host is driven by more than one motivation. In building rapport, knowledge sharing, and insight into local life category, three motivations are identified behind these social practices. However, some hosts had only the altruistic motivation to help others. They can derive value from hosting in CouchSurfing with the least interaction with guest. For this type of host, helping others through providing non-monetary 
accommodation sharing is sufficient as it already provides them with a sense of satisfaction and achievement.

Further, findings on value co-creation practices indicate that hosts perform a central function in their interactions with guests as they act as the primary social contact from the first physical encounter to the end of staying. It can be said that value resulting from interactions during the stay will mostly depend on how hosts practice their motivations. However, the findings also reveal that several resources influence interactions between host and guest in CouchSurfing. These resources are managed by hosts to affect how values are cocreated. Hence, given the importance of these resources in the process of cocreating value, chapter 4.5 will further elaborate these in detail. The discussion will scrutinize which resources affect the interactions and how this influences the host and guest relationship in co-creating value.

\subsection{Resources}

The literature on value co-creation postulates that value is always cocreated through an integration of a set of resources, which influence the way actors live their experience (Johnson \& Neuhofer, 2017; S. L. Vargo et al., 2008). The previous chapter on value co-creation practice has discussed the way actors live their experience in the CouchSurfing context, in which both host and guest have collaborative efforts to co-create value. However, in order to understand what and how value will be derived at the end of the finding section, it is essential to examine through what resources these social interactions between actors are conducted. Therefore, this chapter will now present findings on resources needed to co-create value in the CouchSurfing context and how these resources influence the interaction within.

Discussion on resources relates to research question 3, which asks what resources are needed to co-create value in the CouchSurfing context and how these resources are harnessed in the social interactions to derive value. In detail, this research question will investigate the resources through addressing these following issues: which resources belong to social (e.g., skills and knowledge) or physical (e.g., place/space) categories? How are social and physical resources 
utilised by the host in conducting social interaction? How do these resources affect the interaction between host and guest?

The findings reveal that in the context of CouchSurfing, both social and physical resources are identified affecting social interactions during the stay. Belong to social resources are management strategies and hosts' knowledge. Harnessing these social resources, hosts create efforts to manage the interactions to lead to positive experiences and derive value from numerous interactions. However, falling under physical resource is the CouchSurfing home. As a physical resource, how the CouchSurfing home affects the interactions depends on the effort of the actors, especially host ability to manage and harness it for supporting the interactions.

Subsequently, Figure 4.2 summarises how each resource is harnessed by the host to co-create values during social interactions.

\section{Figure 4.2 Resource for Value Co-creation}

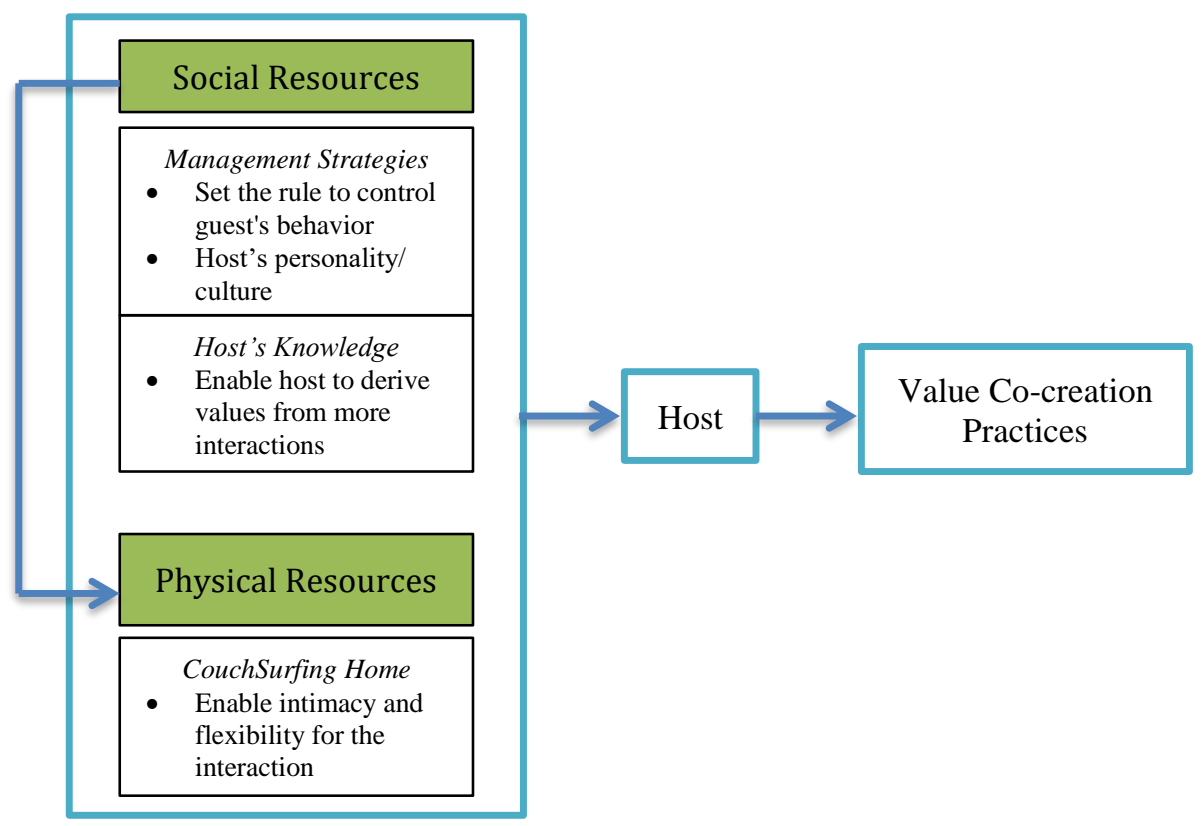

\subsubsection{Management Strategies}

Management strategy in CouchSurfing context is assumed to be a method used by hosts to manage interactions with the guest in order to achieve the expected outcome. In order to do that, findings reveal that hosts will utilise their 
authority as the accommodation owner to set up house rules. While this shows hosts' dominance toward CouchSurfing home, house rules also function as boundaries and guides for guests' dos and don'ts during the stay. In other words, these rules are an approach to control guest behaviour and to create a supportive environment for value co-creation:

I always explain to them my rules. Starting from what time I wake up every day until what time I come back from my work. During those hours, you totally cannot disturb me for something small, unless it is an emergency. (Nadia)

In term of house rule, I am quite flexible but also quite tight ... like curfew ... moreover, you cannot use shoes or sandals at home. (Insan)

In case guests misbehave or disobey the rules, hosts can decide if they want to continue or terminate the social practice. In case they decide to terminate the social practices, as a consequence, the values for both actors will be destroyed. In other words, there is no value co-created or determined during the encounters. This proves that through setting up house rule, hosts possess a significant resource in determining how interactions will proceed and how value will be co-created:

when someone is doing something that I do not like, I will switch my perception to treat him or her professionally (like in paid accommodation). So, instead of having a confrontation, I will tell him or her in politely, or I will just let it be. (Malik)

I sent my guests away eight times because they did not obey my house rule. (Leo)

Further, the findings on management strategies also reveal that hosts' personality is also an important resource to affect the interactions. Personality as a resource directs hosts' thought and behaviour to define which individual or 
environment is compatible to achieving the expected outcome. While house rules manage guests' behaviour during the stay, hosts' personality is more on how hosts behave during the contacts to create a comfortable and supporting environment:

I always try to make my guest feel comfortable around me. I will treat them as a family, close friend, instead of as a guest ... I see myself as an easy going; I do not set any rules. So, I ask my guest to feel at home while staying in my place (Cinta).

Therefore, it can be said that a good personality is a resource for hosts to provide input to create positive interactions. By doing so, they create a more significant probability of leading the interactions into positive experiences, which result in the expected outcomes.

\subsubsection{Host's Knowledge}

Knowledge as a resource enables hosts to have meaningful conversations with guests. Knowing allows hosts to engage in two-way communications. It empowers them with the resource for in-depth conversations while building closeness with the guest and promoting local culture to practice their altruistic behaviour:

I think I am pretty accommodating in the sense of knowledge. In case they want to know what they can do here in Indonesia, or they want to know the characters of local people in general ... Alternatively, they want to have an in-depth conversation with me. I am also available for that kind of conversation. (Insan)

Evidently in value co-creation practice, having knowledge enables hosts to engage in various social practices category. Specifically, from the hosts' perspective, knowledge allows them to promote local culture while giving guests touring company (see Chapter 4.4.1.5). Knowledge also acts as a resource to 
provide guests with tips and advice living in the destination (see Chapter 4.4.2.2) and insight into local life (see Chapter 4.4.3). Therefore, it can be assumed that as a resource, knowledge supports hosts in engaging with guests in every social practice category. To put it another way, knowledge provides hosts with the ability to derive value from numerous interactions.

\subsubsection{Couchsurfing Home}

The CouchSurfing home emerges as the primary physical resource exchange, which characterises the relationship of host and guest. As a physical resource, the impact of the CouchSurfing home toward the interactions depends on how hosts manage and harness it to support social practices. The findings reveal that from a hosts' perspective, CouchSurfing home provides actors with a supporting environment to have in-depth discussions or knowledge sharing. Being in a private setting provides both actors with intimacy for exchanging ideas or even talking about sensitive issues:

the setting is well supported to have an in-depth conversation, something you cannot do at café or any public spaces. Being at home makes me comfortable for talking about something we do not talk with friends or those we often meet. (Malik)

Meanwhile, as part of the local culture, most CouchSurfing homes resemble a local Indonesian house by exterior and standard amenities. For this reason, guests who come from different cultures sometimes have to adjust to these amenities due to unfamiliarity. From the host's perspective, this unfamiliarity can derive a value for them. The cultural difference acts as a bridge for both actors to learn each other's culture, which at the end leading them to cultural exchange and knowledge:

I thought my bathroom is good enough and right according to his [guest from Spain] standard. However, they still asked me a sink for tooth brushing [Indonesian houses usually do not have a sink]. Moreover, when he wanted 
to take a bath, he was confused about the big plastic bucket [in Indonesia, it is usually placed in the bathroom for washing clothes or to collect dirty clothes]. He thought it was a bathtub and used it to take a bath. When he told me that, I was quite surprised before explaining to him how to take a bath in Indonesia. For me, these cultural differences are interesting. (Rio)

However, findings on resources also reveal that this cultural difference may reduce or destroy the value, which was about to be co-created. Guests, who insist on the same standard amenities as their country, usually lead the interactions into a negative experience. By thus, they hinder hosts in co-creating and deriving value from CouchSurfing. As for Nadia, having a demanding guest leads her to conflict with the guest. It brings no value for her but a negative experience:

she complained a lot about the absence of a sink in my house. I already told her many times that it is a type of Indonesian house. However, she insisted that sink was a basic need ... She complained a lot ... I am not happy with the experience. (Nadia)

To sum up, as a physical resource, the CouchSurfing home affects and leads interaction either into a negative or a positive experience depending on how hosts harness or put meaning into it. Positively, the findings reveal the function of CouchSurfing home as a suitable setting to have an in-depth conversation and exchange culture. On the contrary, actors fail to use CouchSurfing as access to provide them with benefit. The characters of the actors, which perceive a CouchSurfing home as an unsatisfactory resource for value co-creation instead of a source of cultural learning, in turn, become generators of value destruction. Therefore, it is also essential to take into consideration that these functions of physical resource emerge as resources because hosts integrate it with social resources and harness it as access to unlocking value in co-creation practices. 


\subsubsection{Conclusion Summary}

Findings on resources identified social and physical resources for the host to co-create value in the CouchSurfing context. Falling under social resources are management strategies and the host's personality, which prove hosts to be the most significant actors in determining value. Through management strategies, hosts manage how guests as their value co-creating partner should behave, while at the same time using their personality to create a supportive environment. In addition to that, knowledge is another vital resource for hosts under the social resource category as it enables them to engage with guests and derive multiple values from numerous interactions. However, the CouchSurfing home emerges as the only physical resource in CouchSurfing. Findings show that the CouchSurfing home as a resource supports interaction by providing an intimate environment for knowledge sharing. However, the impact of CouchSurfing homes toward the interactions depends on how hosts manage and harness it to support social practices.

Overall, hosts harness and integrate these social and physical resources to manage interactions in CouchSurfing into positive experiences. By doing so, hosts can maximize values they are aiming for during social interactions. Therefore, as resources to social interactions have been identified and value cocreation practices have been elaborated upon in Chapter 4.4, the discussion will now move to value outcomes. In order to do this, the following subchapter will present and elaborate in detail how values result from and are determined by the host/guest relationship in CouchSurfing.

\subsection{Value Outcome}

The previous section on resources has elaborated which factors affecting social interaction between hosts and guests and in what way hosts harness these resources in the interactions. Consequently, as value co-creation practice has occurred and a set of resources has been integrated into it, the value will emerge as the outcome (Campos et al., 2015; S. L. Vargo et al., 2008). However, it is also important to note that in value co-creation discourse, actors are the beneficiaries that determine the value for themselves (S. L. Vargo et al., 2008, p. 146). 
Therefore, looking into how actors live their experience and how they perceive it are important factors to identify what values may result. In order to do that, this chapter will now present how values emerge as outcomes from social practices between host and guest in CouchSurfing.

Presentation on value outcome concerns with research question 4, which questioning values hosts derive from hosting in CouchSurfing. In detail, this research question addresses issues of what values are co-created from the host/guest relationship and how values emerge. Further, in order to understand the relationship between original motivation and values as outcomes, this research question examines how they are interrelated and developed during the process of value co-creation (see Table 4.4).

The findings on value outcome show that value seems to emerge only when the interactions between hosts and guests lead to positive experiences. Value does not emerge during the stay only but also after the duration of the stay. Compared to motivation, the finding shows that value outcomes do not develop much during the process of value co-creation. Rapport, knowledge, and altruism appear both as motivation and as value outcome. Meanwhile, job opportunity emerges as the new outcome as a result of knowledge exchange between host and guest. However, although gift emerges in both motivation and value outcome, it shows the different meaning of value in each category.

\section{Table 4.4 Value Outcome}

\begin{tabular}{|c|c|}
\hline Value Outcome & Motivation \\
\hline $\begin{array}{l}\text { Rapport - close friends } \\
\text { - } \quad \text { During the stay } \\
\text { - After the stay }\end{array}$ & $\begin{array}{l}\text { Rapport } \\
\text { - Making a new friend } \\
\text { - The sense of being surrounded by } \\
\text { family } \\
\text { - Mental encouragement and } \\
\text { - } \text { support } \\
\text { - Future expectation }\end{array}$ \\
\hline $\begin{array}{l}\text { Knowledge } \\
\text { • Obtain/ improved skill }\end{array}$ & $\begin{array}{l}\text { Knowledge } \\
\text { - Cultural exchange }\end{array}$ \\
\hline
\end{tabular}




\begin{tabular}{|c|c|}
\hline $\begin{array}{l}\text { - } \quad \text { Limiting the cultural gap } \\
\text { between local and foreigner } \\
\text { - } \quad \text { Being open-minded, confident }\end{array}$ & $\begin{array}{l}\text { - } \text { Travel experience } \\
\text { - Improving language } \\
\text { - Minimizing the cultural gap } \\
\text { - Knowledge transfer }\end{array}$ \\
\hline $\begin{array}{l}\text { Gift } \\
\text { - Symbol of friendship }\end{array}$ & $\begin{array}{l}\text { Gift } \\
\text { - Expected } \\
\text { - Part of introducing local culture }\end{array}$ \\
\hline $\begin{array}{l}\text { Altruism } \\
\text { - Sense of accomplishment }\end{array}$ & $\begin{array}{l}\text { Altruism } \\
\text { - Helping someone } \\
\text { - } \text { Returning favor } \\
\text { - Indirect return } \\
\text { - } \quad \text { Promoting local culture }\end{array}$ \\
\hline $\begin{array}{l}\text { Job Opportunity - new career/ } \\
\text { business opportunity }\end{array}$ & - \\
\hline
\end{tabular}

\subsubsection{Rapport}

Findings on value co-creation practice reveal that rapport is always cocreated together with the guest during encounters (see Chapter 4.4). It can be seen through rapport as a motivation, underlies interactions in every social practice category (see Table 4.2). However, as an outcome, rapport appears during the stay and after the stay. During the stay, the presence of the guest and interactions between both actors create value through a good company and positive experience. Often, the indication for both actors enjoying each other's company can be seen by extending the duration of the stay:

they asked me if they could extend their stay. I said, ok. Additionally, they stayed for a month ... I enjoyed their company. I prefer someone to stay at my place instead of being alone. (Cinta)

Meanwhile, rapport as a value also emerges when both actors maintain a close relationship after the stay. Hosts who derive this value admit that they 
remain close friends with guests, and even develop a relationship like a family and provide support to each other:

we are still in contact up to now ... at least once a week we communicate and chat through a video call. If we have a problem, we reach out to each other. (Insan)

\subsubsection{Knowledge}

Findings on value co-creation practices indicate that hosts practice their knowledge aspiration through every social practice category (see Chapter 4.4). The need for knowledge can be found through activities for building rapport, knowledge sharing, and even insight into local life. Mostly, the transfer of knowledge from guest to host can be seen in their activities, which allow them to share stories and ideas, learning something from each other (see Chapter 4.4.2.3) or through shared learning (see Chapter 4.4.2.4).

However, evidence from value co-creation practice reveal that knowledge as a value may take forms such as new skills or improved skills (see Chapter 4.4.2.3; Chapter 4.4.2.4). In addition to that, knowledge also emerges as a value when contacts with guests from different countries and cultures lead hosts to develop soft skills and confidence. As they gain these values, hosting in CouchSurfing equips them to be more communicative and sociable in dealing with various kinds of people:

hosting in CouchSurfing is like learning soft skills, which I cannot get by studying at university ... it teaches me to interact with people from a different cultural background. (Siwi)

Apart from skills, knowledge also emerges as a value when interactions with guest contribute to developing hosts' cognition and the way of thinking. It may be seen in the way knowledge sharing limits the cultural gap between local and foreigners (see Chapter 4.4.2.1). Bringing together local (host) and foreigner (guest) in a CouchSurfing home encourages each to exchange stories, ideas, and 
culture. Therefore, as they grow understanding and knowledge about each other, they become more open-minded in perceiving differences:

I become an open-minded in understanding religion, politics, any issues, understanding why people do things for a religious reason, and so on.

For hosts who happen to look down on their social and economic condition, interactions with guests bring them a different perspective on how they see themselves. Knowing that other people are interested to know and learn their culture provide them with self-pride and confidence in life:

I cursed myself for being born poor and minority, why was I born in Indonesia? ... They appreciate my culture, impressed by the way I live ... and it grows self-confidence in the way I see myself. (Wahyu)

\subsubsection{Gifts}

Almost half of the participants received a gift and perceived it as their value for hosting in CouchSurfing. Compared to motivation, a gift as a value emerges with a different meaning. In motivation, the host develops gifts as a motivation to introduce culture and as a resource exchange to accommodation sharing (see Chapter 4.3.3). Instead of be willingly given by their guests, gift as a motivation is mandatory for guests to bring.

However, as an outcome, a gift is unexpected. Hosts do not demand it from their guests. It appears as gratitude from guests toward host for letting them stay in the accommodation. Moreover, gift as a value also indicates a positive relationship, which both hosts and guests develop during the stay:

they gave me presents. I never asked, in any case. They gave it to me as a kind gesture. (Insan)

Overall, from the host's perspective, it can be assumed that a gift as a value appears as a token of appreciation from the guest for the positive 
experience, which they both have co-created. Instead of perceived as physical stuff, a gift is more on symbol of friendship between host and guest.

\subsubsection{Altruism}

Altruism as motivation takes form in the way hosts develop a desire to help other people, returning the favour for experience in CouchSurfing, expecting an indirect return and promoting local culture (see Chapter 4.3.1.2). Evidence in the value co-creation practice shows that altruism appears in every category of social practice (see Table 4.3). It drives hosts to build rapport with their guests and practice their altruistic behaviour through welcoming (see Chapter 4.4.1.1) and act as a touring buddy (see Chapter 4.4.1.5).

In knowledge sharing, hosts' altruistic behaviour emerges as help for guests by providing tips and advice in the destination (see Chapter 4.4.2.2). It also drives hosts to give insight into local's lives as part of their devotion toward local culture (see Chapter 4.4.3). Further, findings on value co-creation reveal that some hosts who develop only altruistic motivation for hosting will provide accommodation sharing without requiring any interactions from guests (see 4.4.4).

Therefore, reviewing these findings on motivation and value co-creation practices, altruism as an outcome appears as a sense of accomplishment. By doing these interactions, which are encouraged by the need to practice altruistic behaviour, hosts develop a sense of achievement by providing help and doing something for their environment. By thus, they feel that they have accomplished their urge for hosting in CouchSurfing and gain satisfaction from there.

\subsubsection{Job Opportunity}

Instead of being determined in the original motivation, job opportunity is a new value that emerges from the interactions that occur between host and guest in CouchSurfing. Specifically, job opportunity is identified as a result for sharing ideas during building rapport and knowledge exchange between both actors. As for Avelina, for whom an opportunity came when she realised that she 
had gained knowledge, soft skills, and confidence during her interactions with her guests in CouchSurfing:

I have been a tour guide for 5-6 months. When I stopped selling mixed rice [traditional food from Bali], I gave my surfers a company to explore Bali ... from there I had an idea to start working as a guide. (Avelina)

However, for Priyo, meeting guests from different cultural background allowed him to learn their travelling patterns and behaviour. Using this exploration, he used the opportunity to start and improve his current business in paid accommodation sharing:

mainly, in paid accommodation sharing, they only need Wi-Fi, air conditioner and hot water ... I know their habits ... Learning from them, I have developed many business opportunities. (Priyo)

\subsubsection{Conclusion Summary}

Reviewing original motivation, value co-creation practice, and how resources are integrated into social interactions, values for hosting in CouchSurfing are identified as rapport, knowledge, altruism, gifts, and job opportunity. Understanding these outcomes, it can be concluded that values depend on the way hosts live their experience and how they perceive their experience itself. Therefore, values can be generated from their interactions.

Further, findings reveal that these values only emerge when there are positive interactions between both actors. Concerning original motivation, outcomes from value co-creation practices are a reflection of what hosts want to achieve at the beginning of the stay. As social interactions between both actors proceed, the motivations hosts develop are portrayed through every interaction they conduct. In addition to that, social interactions between host and guest are also affected by how hosts manage and harness their resources to maximise the value they are aiming at. Identified as social and physical resources, hosts play a role in integrating these resources and managing the interactions during 
encounters to result in positive experience to derive the expected values. Often, hosts use these values as motivation to host again in CouchSurfing.

Overall, findings on motivation, value co-creation practices, resources, and value outcome portray a comprehensive explanation to understanding value co-creation in non-monetary accommodation sharing, which has been unclear before this research due to lack of academic contribution. Therefore, the following chapter will now present interpretation and describe the significance of these findings, by providing a revised conceptual framework and briefly explaining answers to each of the research questions. 


\section{Discussion}

\subsection{Introduction}

Findings in chapter 4 provide representation to the host/guest relationship in CouchSurfing context. The chapter sheds light on the value cocreation process, by examining motivation, value co-creation practices, resources, and value as an outcome. Knowing the application of these concepts in CouchSurfing, the interpretation of the findings concerning academic literature will now be discussed.

In presenting the discussion, each part of value co-creation process will be outlined in sequence, starting from motivation, then following on to value cocreation practices, resources, and value. At the beginning of each section, information related to each part will first be revisited in order to understand what has been established in the previous literature. Further, the findings of this research will be discussed and compared to the previous literature to understand how they relate. Accordingly, developed from the original conceptual framework, a revised conceptual framework will be generated at the end of the section, as an illustration, to sum up the entire process and findings of value cocreation in CouchSurfing context (see Figure 5.1).

\subsection{The Motivation for Non-Monetary Accommodation Sharing}

Generally, motivation can be seen as a driving force behind all behaviours (Fodness, 1994). In the study of tourism, discussion on motivation has gained much attention, especially from the perspective of tourists and paid accommodation sharing. However, little has been known concerning motivation from a supply perspective and in non-monetary accommodation sharing.

Therefore, in examining individuals' motivation for hosting in CouchSurfing, this study adopted a social exchange and social capital theory. Social exchange theory provided a basic substance for resource exchange by postulating that individuals were encouraged to enter social interaction as they expected certain rewards, namely love, status, information, goods, money, and services (Blau, 1964; Cropanzano \& Mitchell, 2005). Further, this theory 
postulated that before social interactions, individuals would take a subjective evaluation of reward and cost (Blau, 1964; Cropanzano \& Mitchell, 2005) as a consideration to ensure that cost they spent did not outweigh rewards they aimed at. In addition to that, social exchange theory argued that there is no guarantee of reciprocity of benefits in the exchange (Blau, 1964). Unlike the benefits in the economic exchange, which is formal and contractual, exchange of benefits in the social exchange is a voluntary action and involves unspecified future obligation (Ap, 1992; Blau, 1964). However, although this theory has been widely used in the tourism context, it has mainly focused on the economic benefits (Nunkoo, 2016) and thus has accounted for a lack of research in nonmonetary benefits using the perspective of social exchange theory. Meanwhile, social exchange theory emphasises the importance of individuals' exchange and benefits, while it was important as well to examine the motivation from the perspective of CouchSurfing as a network. Considering these factors, the social capital theory was added in conjunction with social exchange theory.

Further, the social capital theory was used in the research to explain sharing behaviours. The theory assumed that individuals' willingness to share was related to their relationship with the network they were involved in (Nahapiet \& Ghoshal, 1998; Molly McLure Wasko \& Samer Faraj, 2005). Further, this theory explained that sharing behaviour was encouraged by four conditions, namely structural factors, relational factors, cognitive factors, and individual motivation (Molly McLure Wasko \& Samer Faraj, 2005; Molly McLure Wasko \& Samer Faraj, 2005). Structural factors perceived that social interaction and ties between members within the community encouraged them to develop habit of cooperation. Cognitive capital referred to resources that enabled individuals to share. It usually related to individuals' cognitive ability. Subsequently, the relational factor concerned the personal relationship between members, which encouraged individuals towards sharing. Relational factors involve commitment and reciprocity, which arises from repeated interaction and signify a responsibility to be involved in future action (Nahapiet \& Ghoshal, 1998; Molly McLure Wasko \& Samer Faraj, 2005). Therefore, members are eager to help each other within the network as they consider that act as a duty to assist other members and a moral obligation to return work to the network (Wasko et al., 
2005; Molly McLure Wasko \& Samer Faraj, 2005). Lastly, individual motivation refers to other personal encouragements; an individual perceives that their contribution toward others will be worth the effort and their contribution will generate new values (Nahapiet \& Ghoshal, 1998; Molly McLure Wasko \& Samer Faraj, 2005). This motivation may relate to enhancing reputation and enjoyment for helping others (Molly McLure Wasko \& Samer Faraj, 2005).

However, little was known explaining individuals' motivation in nonmonetary accommodation sharing. Although in the study of reciprocity in CouchSurfing (Geiger et al., 2017) provided insight into the motivation to host in CouchSurfing and how it related to pride in the destination and social motives, but it was not adequate to provide a comprehensive overview about the motivation of Indonesian CouchSurfing members to host. In addition to that, none of what has been postulated by social exchange and social capital theory was examined and proven suitable to explain sharing behaviour in nonmonetary accommodation sharing contexts. Therefore, to see the applicability of these theories in non-monetary accommodation sharing contexts, this research asked research question 1: what is the motivation of Indonesian CouchSurfing members for hosting? This research question was concerned with the following issues:

- What rewards are hosts after and why?

- Do they have experience as a guest in CouchSurfing? How do they perceive that?

- How are embedded individuals in the network? How do they perceive CouchSurfing and its members?

Social exchange theory has mentioned five rewards as motivation to enter social interaction in general. However, which rewards contributed to hosting experience in CouchSurfing was unclear. Findings on motivation, therefore, contribute to identifying which rewards are exchanged in CouchSurfing, namely emotional needs (love), knowledge (information), and gifts (goods). Under emotional needs are rapport and altruism, through which individuals harness CouchSurfing to fulfil their needs of friends, company, or practice of their 
altruistic behaviour. Subsequently, there is knowledge and gifts, which allow individuals to exchange information and obtain a present.

Apart from rewards, both findings and the original conceptual framework identify obligation from previous experience as part in individuals' motivation to host. Findings reveal that individuals' experience as a guest provides significance to the decision to host as a contribution to the network. Thus, the finding proves that exchange benefits in the non-monetary platform context involve voluntarily action and future obligation to return to the network as postulated by both social exchange and social capital theory. Further, the contribution to the network is not limited by providing accommodation sharing, but also through the way they treat their guests during encounters.

Meanwhile, as social exchange theory postulates a subjective evaluation of rewards and costs, it was unclear how individuals evaluated the process of decision-making. Especially when rewards and other factors contributing to decision-making were unknown, it was impossible to include this subjective evaluation in the original conceptual framework explicitly. However, this research reveals that in the context of CouchSurfing, this subjective evaluation emerges as the guest selection. It appears as a mechanism for hosts to select their guests based on the needs of their motivations. Two criteria are identified in the process of selecting, namely guests' profile and guests' length of stay. Through this, individuals can consider their compatibility of character with potential guests and estimate the duration of interactions before proceeding to the decision.

Regarding the contribution of CouchSurfing as a network to individuals' motivation, findings show that relational factor contributes to decision-making. It was found that participants with a long tenure of membership in CouchSurfing, who had made positive contacts with other members, tended to harness their relationships in selecting their guest. Simply put, they gave a bigger priority to a request that was recommended by someone they already knew. By so doing, they minimise negative experiences and provide themselves with more significant opportunities to derive values.

However, in terms of the structural factor, findings show that regular contacts and social interaction between members within the community do not 
show significance in the hosting decision. The majority of the participants did not involve themselves in the CouchSurfing as a community. They tended to use CouchSurfing as a personal media to share, without being attached to it as a network. Instead, individuals tended to perceive CouchSurfing differently as a community and CouchSurfing as the only platform they knew, which provided non-monetary accommodation exchange. Thus, this finding provides evidence that as a structural factor, the relationship between individual and CouchSurfing as a network, does not relate to hosting decision.

Meanwhile, the findings did show that cognitive factors exert their influence on motivation. It was found that hosts possessed exclusive knowledge, which allowed them to engage with guests in activities, especially in knowledge sharing (see Chapter 4.4.2). The finding proves that knowledge acted as a cognitive factor that enabled individuals to meet their objective through social interactions. Meanwhile, on top of discussion on CouchSurfing as a network, it seems that individual motivation was more dominant in influencing the hosting decision compare to collective decisions. Findings show that hosting is a personal decision and achievement, encouraged by individual motivation.

In conclusion, this study confirms that to a certain extent social exchange theory is applicable in examining motivation in non-monetary accommodation sharing. The theory contributes to identifying rewards as resource exchanges and provides a fundamental knowledge to formulate a subjective evaluation and future obligation. Due to lack of financial involvement in CouchSurfing, social exchange theory does not provide a comprehensive foundation to exchange occurring in CouchSurfing. Therefore, social capital theory can be used adjacent to the social exchange theory to explain sharing behaviour in CouchSurfing.

Meanwhile, social capital theory provides a foundation in evaluating the contributing factor in hosting decisions. The findings show that the hosting decision is personal, instead of due to a collective network. Therefore, this study only identifies relational factors, cognitive factors, and individual motivation as relevant factors to explain sharing behaviour in CouchSurfing. In other words, it can be concluded that it seems social capital theory does not apply on a community level but more on the individual level in CouchSurfing. 


\subsection{Value Co-Creation Practice}

Chapter 5.2 discussed motivation to host in CouchSurfing, and explained the academic contribution to literature in motivation, especially in the collaborative consumption context. The discussion encompasses factors contributing to' individuals' decision to host, comprised of rewards, an obligation from past action and to what extent that decision related to CouchSurfing as a network. Following on from motivation, this chapter will now interpret the findings on value co-creation practices and how these provide an academic contribution to the discourse of value co-creation practice.

Co-creation in tourism studies can be defined, as "a process of interrelated interactions and activities that connects the tourist and other actors, and experience is the context in which those interactions and activities occur" (Campos et al., 2015, p. 1311). The value in these findings thus emerged as a result of interaction, active participation, and collaborative effort that built upon the experience (Agrawal \& Rahman, 2015; Saarijärvi et al., 2013; S. Vargo \& Lusch, 2004; S. L. Vargo et al., 2008). Therefore, co-creation emphasised the joint value creation with other actors.

Co-creation experience is a process, in which actors influence each other experience to co-create value through interaction and active participation with other subjects. Experiencescape Framework (see Figure 2.1) depicted the process of value co-creation in the tourism setting, in which Campos et al. (2015) portrayed the relationship of the tourist with other actors in building co-creation experiences. In that sphere, tourists are the centres, acting as active subjects, who engage mentally and physically in the interaction and active participation with the environment and other subjects. The value thus emerged as psychological states and process such as sensations, perceptions, images, feeling and emotion.

The study of value co-creation has gained so much attention in tourism studies to depict the relationship between actors to co-create value in a wide range of contexts, including the accommodation sector (Bharwani \& Jauhari, 2013; Cabiddu et al., 2013; P. Chathoth et al., 2013). However, as most empirical research in the study highlights paid accommodation from the perspective of a guest, little attention is paid to hosts in non-monetary accommodation sharing 
and how they derive value from typical accommodation sharing. Hence, in order to fill the gap, this study formulated research question 2: through what practices are values co-created in the host/guest relationship in CouchSurfing context? Some issues considered in this research question are:

- How do hosts interact with guests? Which interactions occur?

- How does motivation influence interaction?

- How do the members see themselves as hosts? What is their role?

While little was gained from previous literature in regards to how values are co-created in non-monetary accommodation sharing, findings of this study identify types of interactions, which characterise host/guest relationship in the CouchSurfing context. These social practices are grouped into four categories, namely building rapport, knowledge sharing, insight into local's life, and least interaction (see Chapter 4). Regarding the spatial dimension of social interaction, the findings show that the process of value co-creation occurs in CouchSurfing homes and local environments, such as tourism spots or hangout places. During these encounters, especially in CouchSurfing homes, it was found that interaction does not occur between host and guest only, but also between guests and hosts' family as the third party.

Findings on value co-creation practices depict how co-creation experiences occur between host and guest in the context of CouchSurfing as a joint value creation (Prahalad \& Ramaswamy, 2004). Identifying the type of social interactions that occurs between both actors, this study shows that the notion of sharing or exchange in CouchSurfing is not limited to accommodation sharing only. Instead, hosting requires individuals to share their time, emotions, and knowledge with guests. This premise explains the finding why hosts make an effort to spend time with their guest during their packed schedule. This finding also aligns with Decrop et al. (2017) and Geiger et al. (2017), who postulated that time is a necessary condition for social practices to occur, and for the value to emerge.

Regarding motivation, in conducting social interactions, hosts are firmly encouraged by the motivation they have. Simply put, hosts conduct social interactions as their effort to accomplish their motivation. Therefore, it can be 
assumed that motivation performs a significant function in shaping the interactions (see Table 4.3). To fulfil the emotional needs of having rapport, for example, hosts are encouraged to build a connection with guests through welcoming, cooking and/or eating together, hanging out together, sharing emotion, and touring with the guest. Similarly, in order to obtain or transfer knowledge, hosts will involve themselves in the sharing of knowledge activities such as deep conversation, providing tips and advice, learning new skills, and shared learning. However, findings found that the actual motivations which underlie each activity are dynamic and do not stem from one motivation only. Therefore, in each social interaction, there is more than one motivation underlying the interactions (see Table 4.3). This finding also aligns with Tourism Career Pattern developed by P. L. Pearce and Lee (2005), which suggested that the individuals' behaviour might be dominated by a set of needs rather than a hierarchical ladder.

Further, findings on value co-creation practices indicate that in such interactions, hosts perform a central function. They act as the primary social contact from the first physical encounter to the end of staying. Regarding value co-creation, hosts see themselves as a responsible actor to manage the interaction with guests during the encounter, so it results in positive experience. Therefore, they create an effort to influence the experience by setting up house rules or harnessing their other resources.

Following up the process of value co-creation, the following section will discuss resources for value co-creation. Knowing the interpretation of the finding on the resource will provide holistic knowledge to analyse value as the outcome of the process of value co-creation.

\subsection{Resources}

Discussion on value co-creation practices show that during the stay, actors are physically and mentally engaged in the interaction with other actors to co-create value. In this stage also, the exchange process between actors occurs in which both actors harness their resources to affect the way they live their experience (Johnson \& Neuhofer, 2017; S. L. Vargo et al., 2008). While the 
discussion on value co-creation practice has been initiated, as a follow up this chapter will now elaborate and interpret findings on resources.

Resources can be defined as "anything that can serve as a source of power in the interaction" (B. Edvardsson \& B. Tronvoll, 2011, p. 294). S. L. Vargo et al. (2008) distinguished resources in value co-creation into social (operant) and physical (operand) resources. Social resources related to social components or human resources such as knowledge and skill. Further, B. Edvardsson and B. Tronvoll (2011) postulated that social resource was dynamic as it acted on other social resources and physical resources. Therefore, Shaw et al. (2011); S. Vargo and Lusch (2004); S. L. Vargo et al. (2008) referred to social resources as an integrator and a key element in the value co-creation. On the other hand, the physical resource is a physical entity (such as place, space, and so on), on which an act was performed to create an effect (Bo Edvardsson \& Bård Tronvoll, 2011; B. Edvardsson \& B. Tronvoll, 2011; S. Vargo \& Lusch, 2004; S. L. Vargo et al., 2008). Put differently, the literature on value co-creation suggested that physical resources only produced effects to co-create value in case of social or human resource acting on or using it. According to this premise, involved resources needed to successfully take action in value co-creation comprised of an integrated entity of social and physical resources (Bo Edvardsson \& Bård Tronvoll, 2011; B. Edvardsson \& B. Tronvoll, 2011; S. Vargo \& Lusch, 2004; S. L. Vargo et al., 2008).

In the hospitality context, Bharwani and Jauhari (2013) postulated that in order to derive value, hospitality employees should have a set of skills needed to deliver an interactive interaction and achieved a significant level of engagement with guest, namely hospitality intelligence (see Chapter 2.3.2.2). Acting as social resource, this hospitality intelligence, which comprised of emotional, cultural, and hospitality experiential intelligence, would equip hospitality employees with competencies and behaviour needed to create emotional connections with guests (Bharwani \& Jauhari, 2013). Emotional intelligence is the ability to harness emotions to guide interpersonal effectiveness and manage emotion to be neutral in a multi-cultural setting. Cultural intelligence is the flexibility of individuals to deal with multicultural situations. Meanwhile, hospitality 
experiential intelligence is the ability gained through experience to solve problems in the hospitality context.

However, in the context of CouchSurfing, which resources were needed to co-create value and how they were harnessed in the interactions were questionable. While P. K. Chathoth et al. (2016); Johnson \& Neuhofer (2017) conducted the study in the context of paid accommodation and from the demand perspective, it was doubted if the context applies to non-monetary accommodation sharing as CouchSurfing. In addition to that, hosting in nonmonetary accommodation sharing was considered as a personal matter encouraged by individual motivation, instead of business driven. Considering the facts, this research provides contribution by examining resources in CouchSurfing and formulates this in research question 3: which resources are needed for these social practices? Some issues to be considered in this research question are:

- Which resources belong to the social and physical categories?

- How do social and physical resources utilized by hosts in conducting social interaction?

- How do these resources affect the interaction between host and guest?

Findings in chapter 4.3 identify resources needed for value co-creation practices in the CouchSurfing context as social and physical resources. Belonging to social resource are management strategies and knowledge. Through management strategies, hosts make an effort to manage the interactions, especially within CouchSurfing home by controlling guest behaviour. In order to do so, hosts set up house rules and create a positive, supportive environment by harnessing their personality. Meanwhile, being knowledgeable is another advantage to conducting value co-creation practice, as knowledge enables hosts to engage with guests in numerous activities. Therefore, knowledge provides the host with the opportunity to derive multiple values from these social practices. On the other hand, a CouchSurfing home emerges as the only physical resource to affect interactions in CouchSurfing. As a resource, it supports interactions by providing an intimate environment for the interactions. However, findings show 
that CouchSurfing homes cannot directly exert their effects on the interactions. Its function in the value co-creation practice depends on the way host harness and put meaning into it.

Findings on resources show that these resources may affect the interaction either in positive or negative ways. Positively, the resource provides the opportunity for hosts to conduct more interactions with guests, which in turn will lead to another value co-creation. Resources enable hosts to anticipate negative encounters with guests through management strategies. However, a CouchSurfing home exemplifies how the resource can negatively affect interactions. As a representation of culture, a CouchSurfing home may destroy the value co-creation process in the way it may cause a cultural gap between actors. Again, it depends on how actors put meaning into and harness this cultural difference as a resource to value co-creation practice.

Reviewing the findings on resources, it can be seen that emotional and cultural intelligence take part in creating a positive interaction between host and guest. These findings are compatible with study conducted by Bharwani and Jauhari (2013) that argued that the ability of hosts to deal and engage with multi-cultural people, their ability to understand cultural differences, and how the react to unexpected encounters are critical for the value to emerge. However, it is argued that the hospitality intelligence framework proposed by Bharwani \& Jauhari (2013) only applies to the extent of emotional and cultural intelligence in CouchSurfing context. Meanwhile experiential hospitality intelligence was not found as a resource in the findings. It is assumed that, as there is no financial involvement in CouchSurfing, both actors develop a different characteristic of hospitality relationship unlike commercial accommodation in Bharwani and Jauhari (2013) study.

Considering how each resource functions to support the interactions, it can be concluded that social resource plays a vital role in value co-creation practices in CouchSurfing. Aligning with the literature, these findings prove that as an actor, the host has a role to control and take action on other resources. The host acts as an integrator of both social and physical resources to successfully affect the interactions. Thus, the finding agrees with S. L. Vargo et al. (2008) that the favourability of co-created experience depends on the ability of actors in 
harnessing resources. Meanwhile as a physical resource, a CouchSurfing home cannot directly exert its influence on the interaction. The finding proves the premise of value co-creation that as a physical resource; it functions as delivery for a host as a social resource to gain benefits (S. L. Vargo et al., 2008).

Knowing resources to social interactions in CouchSurfing context and how they exert their influence on the interactions between hosts and guests provides an illustration that in non-monetary accommodation sharing, social resources place a significant and dominant role. Although physical resources are also identified as dedicated resources, their ability to affect the interaction is minor and depends on the actors as social resources. Therefore, it is assumed that as there is no money involved, the "power of source" (B. Edvardsson \& B. Tronvoll, 2011, p. 294) for the interaction is more on social component and their ability to integrate all these resources.

\subsection{Value Outcome}

Previous sections have provided a comprehensive overview of how value co-creation process occurs in the CouchSurfing context, starting from the original motivations, the social interactions, to a set of resources affected the interactions. Therefore, value as the last part of the sequential process of value co-creation value will now be discussed.

In general, the literature on value co-creation postulated that value emerged from the beneficial use of social resources, which were sometimes performed through physical resources (S. L. Vargo et al., 2008). Value then appears as beneficiaries integrated a set of resources in the value co-creation practice (Schuckert et al., 2017; Shaw et al., 2011; S. L. Vargo et al., 2008). In this consumption process, S. L. Vargo et al. (2008) argued that value was co-created and therefore, experience and perception are crucial in value determination.

Being applied in the study of tourism in monetary accommodation sharing, Johnson and Neuhofer (2017) examined the process of value co-creation between host and guest in an Airbnb context. Although this empirical research delineated value gained by the host, the research was conducted from the perspective of guest with monetary involvement as resource exchange. 
Therefore, these findings may not be applicable in a CouchSurfing context as CouchSurfing is non-monetary accommodation sharing and therefore what value emerges for hosts through CouchSurfing remains vague. Concerning this, this research offers academic contribution by identifying values as outcomes from the host/guest relationship in non-monetary accommodation sharing through research question 4: what value emerges as the outcome for hosting in CouchSurfing? Some issues to be considered in this research question are:

- What values are co-created?

- How do hosts compare values to motivations?

Findings presented in chapter 4.4 identified several values resulting from the host/guest relationship in the CouchSurfing context, notably rapport (love), knowledge (information), gifts (presents), altruism (love), and job opportunity (information). Rapport, knowledge, altruism and gifts were identified as the original motivations of hosts. However, a gift as a value emerges with a different meaning. Instead of expected by the host, gift appears as gratitude from guest toward host, which also can be perceived as a symbol of friendship between actors. Meanwhile, unlike previous values, job opportunity appears as a new value in the co-creation practice.

From the findings, the conclusion can be drawn that motivation provides a significant role in the process of value co-creation to the determination of value itself. Compared to the original motivation, it can be seen that most of the findings on value are the refection to what hosts hope to achieve through hosting experience. This indicates that these motivations to host in CouchSurfing might be values hosts have achieved. Thus, it also explains the function of guest selection to guarantee positive value outcome in the decision-making processes of hosts. Meanwhile, findings show that value also results from the perception of host toward the interactions emerging as new values. Job opportunity and gifts exemplify how value appears as knowledge exchange and building rapport between host and guest. This practice confirms the findings of (Johnson \& Neuhofer, 2017), and their view that co-creation does not inherently have to take place instantly between actors; rather, value can be derived from the effort of a previous activity to encourage another value (Johnson \& Neuhofer, 2017). 
Reflecting on the process of value co-creation in the CouchSurfing context, motivation becomes an integral part of the process. At the beginning of the process, motivations are reflected in every social interaction during host/guest encounters. This means that this interaction between host and guest carries social meaning as they are related to the motivations of hosts toward pursuing rewards they have previously established. Meanwhile, motivations are also reflected in the way hosts apply their resources in the value co-creation practice. Through management strategies, for example, hosts build closeness with guests using their personality and maintaining an enjoyable environment for these interactions. By so doing, hosts can achieve their emotional need of having a rapport. Similarly, with knowledge, using knowledge as their resource, hosts can build connection through having a meaningful conversation or sharing ideas with guests. Therefore, they gain rapport and knowledge at the same time.

Compared to the previous literature on value co-creation, motivation has never been a highlight in the study of value co-creation both in monetary and non-monetary accommodation sharing. The driving force for the actors' behaviour during the encounters has never been explained. Therefore, which resources exert their influence and how these resources are utilised in social interaction have never been examined or understood comprehensively either.

The entire discussion on value emphasises the importance of motivation in the process of value co-creation. As the driving force to share accommodation with other, motivations are reflected in every part of the process. The social meanings of motivations are reflected through the whole process of value cocreation and thus characterise the relationship of host/guest in CouchSurfing. Therefore, it can be concluded that the process of value co-creation in nonmonetary accommodation sharing is a personal decision and socially driven, structured through personal motivation, selecting guests, harnessing resources, and integrating all of the components into value co-creation practice to derive value.

To conclude the discussion chapter, a revised conceptual framework will be presented. This framework will explain and illustrate the interpretation of findings in this chapter, as compared to the original conceptual framework. 


\subsection{A Revised Conceptual Framework}

The previous sections have elaborated the contributions of this thesis to academic literature regarding the process of value co-creation in non-monetary accommodation sharing. Although Chapter 2 provided a comprehensive literature review of the fundamental study in motivation and value co-creation practice in various academic contexts, little was known regarding its applicability in the context of CouchSurfing. Therefore, this research provides a chronological process of value co-creation in the setting by considering motivation as the triggering factor. Further, a revised conceptual framework is presented in Figure 5.1 as an interpretive summary of the research.

The original conceptual framework illustrated fundamental aspects that contribute to analysing the motivation of hosts and the process of value cocreation in CouchSurfing. However, the original conceptual framework also reflected the lack of research in the discourse and confirmed that the previous research was not adequate to explain comprehensively how the process of value co-creation occurred in the CouchSurfing context. Therefore, based on aspects of the original conceptual framework, the revised conceptual framework is generated by adding some aspects based on findings from the research.

Findings on motivation detailed which rewards were exchanged in the host/guest relationship, and validated obligation and network related factors as parts of motivation to host. However, the motivation of the original conceptual framework has been revised by adding guest selection to accommodate the subjective evaluation conducted by hosts before proceeding to the social interactions. Although social exchange theory has postulated this subjective evaluation as part of the motivation (Blau, 1964; Geiger et al., 2017; B. F. Meeker, 1971), it is not known through what mechanism this evaluation is conducted.

When it comes to value co-creation, the original conceptual framework did not mention the type of interactions occurring between host and guest, and how motivation influenced social interaction during the stay. In the revised conceptual framework, these interactions are detailed, and the premise that motivation exerts its influence in social interaction is validated. Similarly, the original conceptual framework assumed that social and physical resources influenced the interaction through the action of hosts. Which resource belonged 
to which category - social or physical resources - was not clear, and therefore this revised conceptual framework emerges includes this detail from the research. In addition to that, the original conceptual framework did not show the connection between motivation and resource, while in the revised conceptual framework, motivation is linked to resources to accommodate the premise that the way hosts use their resources is also affected by the motivation they have.

The last part of both the original and revised conceptual frameworks is value outcome. The revised conceptual framework adds and details value as the outcome from the process of value co-creation. The interrelated relationship between value and motivation proposed by the original conceptual framework is validated in the revised conceptual framework. This shows that value emerges as the reflection of the motivation, while at the same time, value can act as motivation for individuals to host in the future. In addition to that, the revised conceptual framework links value to guest selection, to accommodate the premise that hosts will consider their previous experiences of value co-creation and select guests based on their experience to guarantee a positive outcome.

To sum up, a revised conceptual framework shows the contribution of this research to academic literature, through a sequential process of value cocreation in non-monetary accommodation sharing. By including motivation as an encouraging factor to host, this research provides a holistic analysis of the value co-creation process, by proving that the entire process of value co-creation is a matter of personal choice. 
Figure 5.1 Revised conceptual framework of Value Co-creation in CouchSurfing

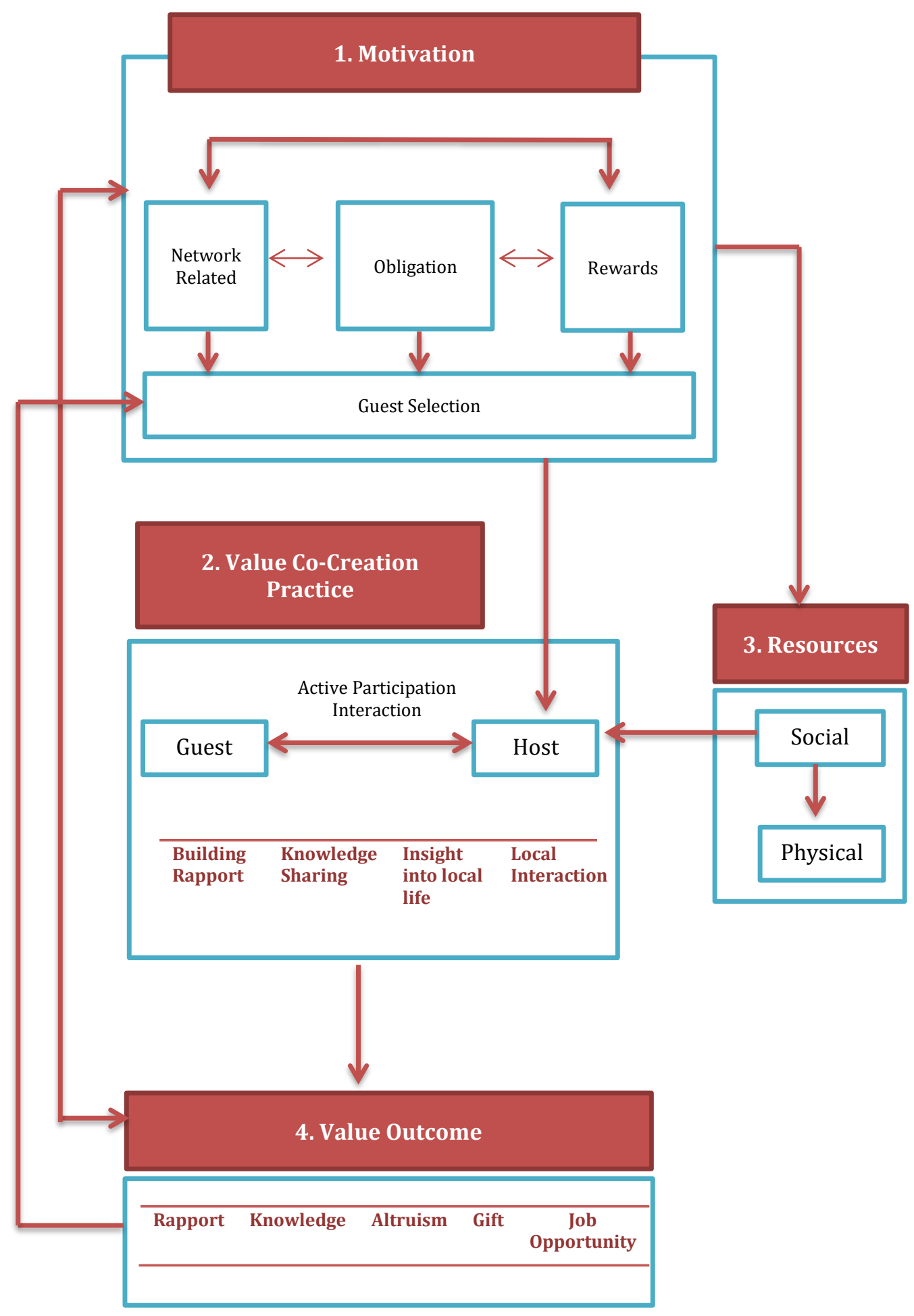




\section{Conclusion}

\subsection{Summary of the Study}

This research aimed to examine social interactions in non-monetary accommodation sharing through the perspective of hosts based upon the concept of value co-creation. Four research questions (see Chapter 1.3) were formulated to guide the analysis of the study and address the research gaps identified in the literature. This concluding chapter will begin with a brief review and answer of these research questions to provide a summary of the study.

\section{What is the motivation of Indonesian CouchSurfing members to host?}

The findings on motivation identified three contributing factors that encouraged individuals to host, namely rewards, obligation, and network related matters. Reviewing these factors, individuals decided to host predominantly for personal reasons. Although the decision may relate to a specific network, it was found that this was relevant not on a CouchSurfing community level, but more on an individual level. This premise also highlights that hosting in CouchSurfing is a personal act instead of a collective decision.

The research also found that, before deciding to engage in social interactions, hosts conduct a 'guest selection' to choose the criteria for their guests. This part of the motivation is considered a subjective evaluation to secure a positive outcome and a mechanism to maximise the co-created value. Further, discussion on motivation reveals that motivation plays a crucial role in value cocreation process in CouchSurfing interactions. Motivation is reflected in the way hosts conduct social interactions, utilise their resources, and determine the outcome.

\section{Through what practices is value co-created between host and guest in a CouchSurfing context?}

Previous literature mostly focused on value co-creation in monetary accommodation sharing and neglected the non-monetary accommodation context. Therefore, little was known regarding the process of value co-creation in non-monetary accommodation sharing. 
By examining the practices of value co-creation, this research identified the types of social interactions that characterise the host/guest relationship in CouchSurfing exchanges from the hosts' perspective. The findings show that value co-creation practices through CouchSurfing are conducted through building rapport, knowledge sharing, and insight into local life, and even occur with little personal interaction

\section{Which resources are required for these social practices?}

In order to co-create value, the integration of a set of resources is needed (S. L. Vargo et al., 2008). Findings reveal that social resources appear as management strategies and knowledge, while physical resources emerge as the hosts' CouchSurfing home. Applied in the interaction, the research confirms the previous literature on value co-creation, i.e., that social resources play an essential role in integrating and harnessing all other resources (Campos et al., 2015; Johnson \& Neuhofer, 2017; Schuckert et al., 2017; S. L. Vargo et al., 2008). Meanwhile, physical resources function as a vehicle for the social resource to unlock their value (Campos et al., 2015; Johnson \& Neuhofer, 2017; Schuckert et al., 2017; S. L. Vargo et al., 2008). Therefore, a CouchSurfing home's function will depend on the host to manage and harness for the interaction.

\section{What values emerge as outcomes for hosting in CouchSurfing?}

In general, the literature on value co-creation postulated that value emerged from the beneficial use of social resources, which were sometimes performed through physical resources (Campos et al., 2015; S. L. Vargo et al., 2008). The findings identified several values resulting from the host/guest relationship in the CouchSurfing context, specifically rapport (love), knowledge (information), gifts (presents), altruism (love, and job opportunity (information). Rapport, knowledge, altruism and gift were identified as the original motivations of hosts. However, a gift as a value emerges with a different meaning from the original motivation. Instead of something expected by the host, a gift appears as gratitude from guest toward host, which also can be perceived as a symbol of friendship between actors. Meanwhile, unlike previous values, job opportunity appears as a new value in the co-creation practice. 
However, the entire discussion on value emphasises the importance of motivation in the process of value co-creation. Motivations are reflected in every phase of the process and thus characterise the relationship of host-guest in CouchSurfing. Meanwhile, motivation is interrelated with value, meaning that hosts can use value as their motivation for hosting in the future. In addition to that, the host also relates value to guest selection as an effort or standard to guarantee the outcome.

\subsection{Limitation and Future Research}

This research has some limitations in both the methodology and the findings, which can be briefly summarized as follows:

1) The resources of this research did not allow for a sampling design that took into account the geographical distribution of CouchSurfing hosts across Indonesia. Consequently, the numbers of participants in each region are not equal in number and do not reflect the wider population. $50 \%$ of the participants are from Bali, while the rest are from Yogyakarta and Bandung. It is assumed that the findings will mostly reflect the hosting experience in these select regions, and neglect CouchSurfing hosting experience in other regions that take place within different environmental and societal contexts. Therefore, the applicability of findings to other areas in Indonesia should be done with caution.

2) Having Indonesian CouchSurfing members as the respondents, the findings of the research strongly reflected upon Indonesian culture and non-western perspectives. Therefore, transferring the results of this research to another cultural context, especially western, may come with limitations reflected especially in the motivations, social interactions and resources. 
In order to address these limitations and gaps, future research could be conducted as follows:

1) While the findings of the research reflect certain touristic regions in Indonesia, the perspective from less touristic regions is needed. With fewer international and domestic tourist arrivals in a region, it would be insightful to know how the process of value co-creation occurs and what value hosts or the destination can derive.

2) While this research takes the hosts' perspective in the value co-creation, how and what values guests desire and derive is needed as a counter perspective to provide a comprehensive and holistic picture of value cocreation in non-monetary accommodation sharing.

3) Section $\underline{1.4 .1}$ has delineated that Indonesia was a country with at least 1,300 ethnic groups and thus indicates there is no generalised Indonesian culture. The future research should see if hosts' culture has impacted and reflected in the hosting behaviour, the way hosts treat their guests.

4) In addition, research on value co-creation based on gender can be considered in the future. Although the participants of the research show an equal gender distribution, the research does not accommodate gender perspective in the findings. Bearing in mind that gender-based social pressure occurs in many sectors in Indonesia, social interactions conducted by both genders might signify different values.

\subsection{Implications}

The primary objective of the research was to understand how social interaction occurs in non-monetary accommodation sharing, by investigating the motivation and value co-creation from the hosts' perspective. In order to provide the theoretical foundation in conducting the research, the literature review in section 2 has elaborated relevant theory on motivation and value co-creation. Meanwhile, the findings show that by providing non-monetary accommodation sharing, hosts achieve their goals in their original motivation and also maintain and derive new value from their interactions with guests. Therefore, reviewing 
the entire process of the research, practical and theoretical implications are identified, which will be presented in the following sections.

\subsubsection{Theoretical implication}

This research provides theoretical implications for the notion of collaborative consumption. By situating itself within a non-monetary collaborative consumption context, this research provides a comprehensive description of what encourages individuals to function as hosts. While in the previous literature the motivation to join non-monetary collaborative platforms was unclear, this research provides an understanding that instead of being business-driven, the motivation of hosting in CouchSurfing is solely based on personal and emotional reasons.

Further, this research provides a theoretical implication for value cocreation. Previous research on value co-creation did not elaborate on the process of value co-creation in the context of non-monetary collaborative consumption and what value hosts can derive. The findings of this research provide a theoretical contribution to how social interactions occur between host and guest and further examine what value hosts can derive by providing free accommodation sharing. In addition to that, the findings of this research reveal that hosts' motivations play a significant role throughout the whole process of value co-creation. Motivation exerts its influence in the way hosts select their guests, use and harness their resources, and determine the outcome. While previous research on value co-creation neglects motivation in the process of value co-creation, inserting motivation into the process, this research provides a theoretical contribution and a comprehensive investigation toward co-created value in the context of non-monetary accommodation sharing.

\subsubsection{Practical implications}

CouchSurfing provides the opportunity for both host and guest to meet directly and conduct social interactions. In co-creating experiences, both actors are involved in activities, which influence their respective experiences. This intimacy and knowledge sharing between actors may elevate the emotional 
engagement, and thus provide destination marketing with an opportunity to promote tourism by offering an authentic experience of living in the destination. In order to do that, marketing managers can capture the intimacy between host and guest into the marketing campaign to show how destinations offer emotional and cognitive engagement in the experience. By visiting the destination, tourists can derive not only personal connections as value, but also meeting with like-minded people, the opportunity to increase knowledge, gain new skills and insight into other people's lives, culture and experiences. In addition to that, this research provides an insight for marketing managers into an innovative approach to provide memorable experiences for tourists by partnering with hosts to encourage active participations with guests.

Aside from marketing, destination managers can harness the host/guest relationship to improve the skills of the residents or create job opportunities. Findings show that by hosting, individuals are involved in knowledge sharing activities. Hosts can increase or gain new knowledge and skills and positive selfidentity, which will be helpful in the development of tourism destination. Meanwhile, for paid accommodation, the relationship between host and guest in CouchSurfing can be an insight to build intimacy and provide authentic experience for guests to create emotional bonding and increase financial benefits. Training employees to build emotional and cognitive connections may improve the degree of host/guest relationship and thus impact on experience memorability.

However, for the CouchSurfing platform, this research may contribute to creating guidelines for its members regarding do's and don'ts while staying in Indonesia. For hosts, this guideline is an insight to harness CouchSurfing as an opportunity to achieve their personal goals and which interactions they can conduct to achieve the motivation. For guests, this guideline provides an insight into Indonesian hosts' culture and expectations in Indonesia. Understanding the culture and expectation, the guideline will minimise the negative encounters between host and guest. For the CouchSurfing platform, as the negative encounters between host and guest can be minimised, it may encourage more people to join CouchSurfing and make payment to verify their membership. While the CouchSurfing platform is financially promoted, the mission of 
CouchSurfing to enable people to meet new acquaintances and make connections through the platform will also be successfully achieved. 


\section{Bibliography}

Agrawal, A. K., \& Rahman, Z. (2015). Roles and Resource Contributions of Customers in Value Co-creation. International Strategic Management Review, 3(1-2), 144-160. doi:10.1016/j.ism.2015.03.001

Andriotis, K., \& Vaughan, R. D. (2016). Urban Residents' Attitudes toward Tourism Development: The Case of Crete. Journal of Travel Research, 42(2), 172-185. doi:10.1177/0047287503257488

Ap, J. (1992). Residents' perceptions on tourism impacts. Annals of Tourism Research, 19(4), 665-690. doi:10.1016/0160-7383(92)90060-3

Argyle, M. (1981). Social situations / Michael Argyle, Adrian Furnham, Jean Ann Graham. Cambridge, U.K.: Cambridge, U.K. : Cambridge University Press.

$\mathrm{Au}, \mathrm{K}$. H. (1998). Social constructivism and the school literacy learning of students of diverse backgrounds. Journal of literacy research, 30(2), 297 319.

Becker, G. (1975). Human capital : A theoretical and empirical analysis (2nd ed.). New York: Columbia University Press.

Belk, R. (2014). You are what you can access: Sharing and collaborative consumption online. Journal of Business Research, 67(8), 1595-1600. doi:10.1016/j.jbusres.2013.10.001

Bharwani, S., \& Jauhari, V. (2013). An exploratory study of competencies required to co - create memorable customer experiences in the hospitality industry. International Journal of Contemporary Hospitality Management, 25(6), 823-843. doi:10.1108/ijchm-05-2012-0065

Blau, P. (1964). Exchange and power in social life. New York: J Willey.

Botsman, R., \& Rogers, R. (2010). What's mine is yours. The rise of collaborative consumption.

BPS. (2018). Number of Foreign Visitor to Bali by Gate, 2009-2018

Retrieved from

https://bali.bps.go.id/statictable/2018/03/05/46/jumlah-wisatawanasing-ke-bali-menurut-pintu-masuk-2009-2018.html

Braun, V., \& Clarke, V. (2006). Using thematic analysis in psychology. Qualitative Research in Psychology, 3(2), 77-101. doi:10.1191/1478088706qp063oa

Burr, V. (2015). Social Constructionism: Taylor and Francis.

Cabiddu, F., Lui, T.-W., \& Piccoli, G. (2013). Managing Value Co-Creation in the Tourism Industry. Annals of Tourism Research, 42, 86-107. doi:10.1016/j.annals.2013.01.001

Camilleri, J., \& Neuhofer, B. (2017). Value co-creation and co-destruction in the Airbnb sharing economy. International Journal of Contemporary Hospitality Management, 29(9), 2322-2340. doi:10.1108/ijchm-09-20160492

Campos, A. C., Mendes, J., do Valle, P. O., \& Scott, N. (2016). Co-Creation Experiences: Attention and Memorability. Journal of Travel \& Tourism Marketing, 33(9), 1309-1336. doi:10.1080/10548408.2015.1118424

Campos, A. C., Mendes, J., Valle, P. O. d., \& Scott, N. (2015). Co-creation of tourist experiences: a literature review. Current Issues in Tourism, 1-32. doi:10.1080/13683500.2015.1081158 
Cavana, R., Delahaye, B., \& Sekaran, U. (2001). Applied business research : Qualitative and quantitative methods / Robert Y. Cavana, Brian L. Delahaye, Uma Sekaran. Milton, Qld: J. Wiley.

Chathoth, P., Altinay, L., Harrington, R. J., Okumus, F., \& Chan, E. S. W. (2013). Coproduction versus co-creation: A process based continuum in the hotel service context. International Journal of Hospitality Management, 32, 1120. doi:10.1016/j.ijhm.2012.03.009

Chathoth, P. K., Ungson, G. R., Harrington, R. J., \& Chan, E. S. W. (2016). Cocreation and higher order customer engagement in hospitality and tourism services. International Journal of Contemporary Hospitality Management, 28(2), 222-245. doi:10.1108/ijchm-10-2014-0526

Chen, D.-J. (2012). Global concept, local practice: Taiwanese experience of CouchSurfing. Hospitality \& Society, 1(3), 279-297. doi:10.1386/hosp.1.3.279_1

Coleman, J. S. (1990). Foundations of social theory / James S. Coleman. Cambridge, Mass.: Cambridge, Mass. : Belknap Press of Harvard University Press.

Constant, D., Sproull, L., \& Kiesler, S. (1996). The kindness of strangers: The usefulness of electronic weak ties for technical advice. Organization science, 7(2), 119-135.

Couchsurfing. (2017). Retrieved from www.couchsurfing.com

Couchsurfing. (2018). Retrieved from www.couchsurfing.com

Creswell, J. W. (2013). Qualitative inquiry and research design: Choosing among five designs: Thousand Oaks, CA: Sage.

Cropanzano, R., \& Mitchell, M. S. (2005). Social Exchange Theory: An Interdisciplinary Review. Journal of Management, 31(6), 874-900. doi:10.1177/0149206305279602

Cropanzano, R., \& Mitchell, M. S. (2016). Social Exchange Theory: An Interdisciplinary Review. Journal of Management, 31(6), 874-900. doi:10.1177/0149206305279602

Decrop, A., Del Chiappa, G., Mallargé, J., \& Zidda, P. (2017). “Couchsurfing has made me a better person and the world a better place": the transformative power of collaborative tourism experiences. Journal of Travel \& Tourism Marketing, 35(1), 57-72. doi:10.1080/10548408.2017.1307159

Ecclestone, K., \& Field, J. (2003). Promoting Social Capital in a 'Risk Society'- a new approach to emancipatory learning or a new moral authoritarianism? British Journal of Sociology of Education, 24(3), 267282.

Edvardsson, B., \& Tronvoll, B. (2011). Value Co-Creation and Value-in-Context: Understanding the Influence of Duality of Structures. Paper presented at the Service Sciences (IJCSS), 2011 International Joint Conference on.

Edvardsson, B., \& Tronvoll, B. (2011, 25-27 May 2011). Value Co-Creation and Value-in-Context: Understanding the Influence of Duality of Structures. Paper presented at the 2011 International Joint Conference on Service Sciences.

Ek, R., Larsen, J., Hornskov, S. B., \& Mansfeldt, O. K. (2008). A Dynamic Framework of Tourist Experiences: Space - Time and Performances in the Experience Economy. Scandinavian Journal of Hospitality and Tourism, 8(2), 122-140. doi:10.1080/15022250802110091 
Flora, C. B. (2004). Community dynamics and social capital. Agroecosystems analysis(agroecosystemsa), 93-107.

Fodness, D. (1994). Measuring tourist motivation. Annals of Tourism Research, 21(3), 555-581. doi:10.1016/0160-7383(94)90120-1

Galletta, A. (2013). Mastering the semi-structured interview and beyond : from research design to analysis and publication / Anne Galletta

foreword by William E. Cross, Jr. In W. E. Cross (Ed.): New York : New York University Press.

Geiger, A., Horbel, C., \& Germelmann, C. C. (2017). “Give and take”: how notions of sharing and context determine free peer-to-peer accommodation decisions. Journal of Travel \& Tourism Marketing, 35(1), 5-15. doi:10.1080/10548408.2016.1231101

Getz, D. (1986). The tourism system: An introductory text: By Robert Christie Mill and Alastair M. Morrison, Prentice-Hall, Inc. (Englewood Cliffs, NJ 07632, USA) ISBN 0-13-925645-8, 1985, XX + 457 pp. (tables, illustrations, index) $\$ 25.95$ (cloth). Annals of Tourism Research, 13(1), 143-146. doi:10.1016/0160-7383(86)90070-8

Goeldner, C. R. (2009). Tourism principles, practices, philosophies / Charles R. Goeldner, J.R. Brent Ritchie. In J. R. B. Ritchie \& C. Ebooks (Eds.), (Eleventh ed.. ed.). Hoboken, N.J.: Hoboken, N.J. : John Wiley.

Guttentag, D. (2015). Airbnb: disruptive innovation and the rise of an informal tourism accommodation sector. Current Issues in Tourism, 18(12), 11921217. doi:10.1080/13683500.2013.827159

Haley, A. J., Snaith, T., \& Miller, G. (2005). The social impacts of tourism a case study of Bath, UK. Annals of Tourism Research, 32(3), 647-668. doi:10.1016/j.annals.2004.10.009

Hamari, J., Sjöklint, M., \& Ukkonen, A. (2016). The sharing economy: Why people participate in collaborative consumption. Journal of the Association for Information Science and Technology, 67(9), 2047-2059.

Hammersley, M. (2013). What is Qualitative Research? London: Bloomsbury Academic.

Hellwing, K., Morhart, F., Kocher, B., \& Zisiadis, G. (2014). Share Your Life and Get More of Yourself. Experience Sharing in Couchsurfing. Advances in Consumer Research, 42, 510.

Hippel, E. V., \& Krogh, G. V. (2003). Open source software and the "privatecollective" innovation model: Issues for organization science. Organization science, 14, 2.

Holtz, G. (2014). Generating social practices. Journal of Artificial Societies and Social Simulation, 17(1), 17.

Homans, G. C. (1961). Social behavior. New York: Harcourt, Brace and World.

Homans, G. C. (1961). Social behaviour : its elementary forms / by George Caspar Homans. New York: New York : Harcourt, Brace.

Hsieh, C.-M., Tsai, B.-K., \& Chen, H.-S. (2017). Residents' Attitude toward Aboriginal Cultural Tourism Development: An Integration of Two Theories. Sustainability, 9(6). doi:10.3390/su9060903

Huysman, M., \& Wulf, V. (2004). Social Capital and Information Technology. Cambridge, MA: MIT Press. 
Huysman, M., \& Wulf, V. (2005). IT to support knowledge sharing in communities, towards a social capital analysis. Journal of Information Technology, 21(1), 40-51. doi:10.1057/palgrave.jit.2000053

Johnson, A.-G., \& Neuhofer, B. (2017). Airbnb - an exploration of value cocreation experiences in Jamaica. International Journal of Contemporary Hospitality Management, 29(9), 2361-2376. doi:10.1108/ijchm-08-20160482

Jones, S. (2005). Community-Based Ecotourism. Annals of Tourism Research, 32(2), 303-324. doi:10.1016/j.annals.2004.06.007

Kim, S., Lee, K. Y., Koo, C., \& Yang, S.-B. (2017). Examining the influencing factors of intention to share accommodations in online hospitality exchange networks. Journal of Travel \& Tourism Marketing, 35(1), 16-31. doi:10.1080/10548408.2016.1244024

Lampinen, A., \& Cheshire, C. (2016). Hosting via Airbnb. Paper presented at the Proceedings of the $2016 \mathrm{CHI}$ Conference on Human Factors in Computing Systems - CHI ' 16.

Leana III, C. R., \& Van Buren, H. J. (1999). Organizational social capital and employment practices. Academy Of Management Review, 24(3), 538-555.

Leinbach, T. R., Mohamad, G. S., Wolters, O. W., \& Adam, A. W. (2019). Indonesia Britannica: Encyclopædia Britannica, inc.

Lewicki, R. J., \& Bunker, B. B. (1996). Developing and maintaining trust in work relationships. Trust in organizations: Frontiers of theory and research, 114, 139.

Lusch, R. F., \& Vargo, S. L. (2006). Service-dominant logic: what it is, what it is not, what it might be The Service-Dominant Logic of Marketing (pp. 6174): Routledge.

Marwell, G., Oliver, P. E., \& Prahl, R. (1988). Social Networks and Collective Action: A Theory of the Critical Mass. III. American Journal of Sociology, 94(3), 502-534. doi:10.1086/229028

Meeker, B. F. (1971). Decisions and exchange. American Sociological Review, 485495.

Meeker, B. F. (1971). Decisions and Exchange. American Sociological Review, 36(3), 485-495. doi:10.2307/2093088

Minichiello, V. (2008). In-depth interviewing : principles, techniques, analysis / Victor Minichiello, Rosalie Aroni, Terrence Hays (3rd ed.. ed.). Sydney: Sydney : Pearson Education Australia.

Molm, L. (2003). Theoretical comparisons of forms of exchange. Sociol. Theor., 21(1), 1-17.

Molm, L. D. (1994). Dependence and risk: transforming the structure of social exchange. (Special Issue: Conceptualizing Structure in Social Psychology ). Social Psychology Quarterly, 57(3), 163. doi:10.2307/2786874

Molz, J. G. (2012). CouchSurfing and network hospitality: 'It's not just about the furniture'. Hospitality \& Society, 1(3), 215-225.

doi:10.1386/hosp.1.3.215_2

Moscardo, G. (2014). Social capital, trust and tourism development.

Moscardo, G., Konovalov, E., Murphy, L., McGehee, N. G., \& Schurmann, A. (2017). Linking tourism to social capital in destination communities. Journal of 
Destination Marketing \& Management, 6(4), 286-295.

doi:10.1016/j.jdmm.2017.10.001

Moscardo, G., Morrison, A. M., Pearce, P. L., Lang, C.-T., \& O'Leary, J. T. (1996).

Understanding vacation destination choice through travel motivation and activities. Journal of Vacation Marketing, 2(2), 109-122.

doi:10.1177/135676679600200202

Myers, M. D. (2009). Qualitative research in business and management / Michael D. Myers. Los Angeles

London: Los Angeles

London : SAGE.

Nahapiet, J., \& Ghoshal, S. (1998). Social capital, intellectual capital, and the organizational advantage. Academy of Management Review, 23(2), 242266.

Neuhofer, B., Buhalis, D., \& Ladkin, A. (2012). Conceptualising technology enhanced destination experiences. Journal of Destination Marketing \& Management, 1(1-2), 36-46.

Nunkoo, R. (2016). Toward a more comprehensive use of social exchange theory to study residents' attitudes to tourism. Procedia Economics and Finance, 39, 588-596.

Paraskevaidis, P., \& Andriotis, K. (2017). Altruism in tourism: Social Exchange Theory vs Altruistic Surplus Phenomenon in host volunteering. Annals of Tourism Research, 62, 26-37. doi:10.1016/j.annals.2016.11.002

Pearce, D. G. (2012). Frameworks for tourism research / Douglas Pearce. Wallingford, Oxfordshire

Cambridge, MA: Wallingford, Oxfordshire

Cambridge, MA : CABI.

Pearce, P. L. (1998). Tourism : bridges across continents / Alastair Morrison, Philip Pearce, Joy Rutledge. Sydney: Sydney : McGraw-Hill.

Pearce, P. L., \& Lee, U.-I. (2005). Developing the Travel Career Approach to Tourist Motivation. Journal of Travel Research, 43(3), 226-237. doi:10.1177/0047287504272020

Pietilä, O. (2011). CouchSurfers' motivations to host travelers in Spain.

Prahalad, C. K., \& Ramaswamy, V. (2004). Co-creation experiences: The next practice in value creation. Journal of Interactive Marketing, 18(3), 5-14. doi:10.1002/dir.20015

Putnam, R. D. (1995). Tuning In, Tuning Out: The Strange Disappearance of Social Capital in America. PS: Political Science and Politics, 28(4), 664-683. doi:10.2307/420517

Rihova, I., Buhalis, D., Moital, M., \& Gouthro, M.-B. (2015). Conceptualising Customer-to-customer Value Co-creation in Tourism. International Journal of Tourism Research, 17(4), 356-363. doi:10.1002/jtr.1993

Rosen, D., Lafontaine, P. R., \& Hendrickson, B. (2011). CouchSurfing: Belonging and trust in a globally cooperative online social network. New Media \& Society, 13(6), 981-998.

Saarijärvi, H., Kannan, P. K., \& Kuusela, H. (2013). Value co-creation: theoretical approaches and practical implications. European Business Review, 25(1), 6-19. doi:10.1108/09555341311287718 
Schuckert, M., Peters, M., \& Pilz, G. (2017). The co-creation of host-guest relationships via couchsurfing: a qualitative study. Tourism Recreation Research, 1-15. doi:10.1080/02508281.2017.1384127

Sekaran, U. (2010). Research methods for business : a skill-building approach / Uma Sekaran and Roger Bougie (5th ed.. ed.). Chichester: Chichester : Wiley.

Shaw, G., Bailey, A., \& Williams, A. (2011). Aspects of service-dominant logic and its implications for tourism management: Examples from the hotel industry. Tourism Management, 32(2), 207-214. doi:10.1016/j.tourman.2010.05.020

Svendsen, G. L. H. (2006). Studying social capital in situ: A qualitative approach. Theory and Society, 35(1), 39-70. doi:10.1007/s11186-006-6780-3

Tussyadiah, I. P., \& Pesonen, J. (2016). Impacts of Peer-to-Peer Accommodation Use on Travel Patterns. Journal of Travel Research, 55(8), 1022-1040. doi:10.1177/0047287515608505

Urry, J. (2002). The tourist gaze / John Urry (2nd ed.. ed.). London: London : SAGE.

Vargo, S., \& Lusch, R. (2004). Evolving to a new dominant logic for marketing J. Mark. (Vol. 68, pp. 1-17).

Vargo, S. L., Maglio, P. P., \& Akaka, M. A. (2008). On value and value co-creation: A service systems and service logic perspective. European Management Journal, 26(3), 145-152. doi:10.1016/j.emj.2008.04.003

Waitt, G. (2003). Social impacts of the Sydney Olympics. Annals of Tourism Research, 30(1), 194-215. doi:10.1016/s0160-7383(02)00050-6

Ward, C., \& Berno, T. (2011). Beyond social exchange theory. Annals of Tourism Research, 38(4), 1556-1569. doi:10.1016/j.annals.2011.02.005

Wasko, McLure, M., \& Faraj, S. (2005). Why should I share? Examining social capital and knowledge contribution in electronic networks of practice. MIS Quarterly, 29(1), 35-37.

Wasko, M. M., \& Faraj, S. (2000). "It is what one does": why people participate and help others in electronic communities of practice. The journal of strategic information systems, 9(2-3), 155-173.

Wasko, M. M., \& Faraj, S. (2005). Why should I share? Examining social capital and knowledge contribution in electronic networks of practice. MIS Quarterly, 35-57.

Wasko, M. M., \& Faraj, S. (2005). Why should I share? Examining social capital and knowledge contribution in electronic networks of practice. MIS Quarterly, 29(1), 35-37.

Wilson, D. S. (2015). Does Altruism Exist?: Culture, Genes, and the Welfare of Others: Yale University Press.

WTTC. (2018). Travel and tourism economic impact: Indonesia 2018. Retrieved from https://www.wttc.org/-/media/files/reports/economic-impactresearch/countries-2018/indonesia2018.pdf 


\section{Appendices}

Appendix A: Information sheet for participants

Appendix B: Information sheet for focus group

Appendix C: Consent form

Appendix D: Consent form for Focus Group

Appendix E: Interview Guidelines

Appendix F: Interview Guidelines for Focus Group 


\title{
Appendix A: Information sheet for participants
}

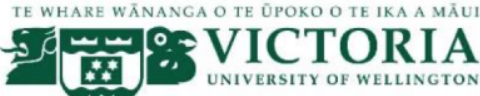 \\ Social Interaction in Non-Commercial Accommodation Sharing: Host's Motivation and Value Co- \\ Creation
}

\section{Information Sheet for Participants}

Dear participant,

Thank you for showing an interest in this project. My name is Undhan Sevisari and I am currently preparing my master of Tourism Management thesis at the School of Management at Victoria University of Wellington. I would like to invite you to take part in my research project. Please read this information sheet carefully before deciding whether or not to participate. If you decide to participate I thank you and please sign the attached consent form. Otherwise, if you decide not to participate, I thank you for considering my request.

The aim of the project:

As part of my thesis project, I am looking at social interactions among host and guest in noncommercial's accommodation in Indonesia. Further, I am examining the motive of the host participating in CouchSurfing activities and how social interaction between host and guest derives values for hosts.

\section{How can you help?}

Participation in this project involves a one-on-one interview with the researcher (myself), at a time and place that would be convenient to you. The interview will be recorded and take approximately 30-45 minutes but will only begin with your consent. Please be aware that you may decline to answer any particular questions and that you may withdraw from participation in the project at any time. You also have the opportunity to withdraw your participation until Monday, $17^{\text {th }}$ September 2018 , either by phone, email or mail. In this case, the information provided will be destroyed.

\section{What will happen to information you give?}

The interview recording and transcripts will be securely stored on a password-protected computer. Only my supervisor and myself will have access to it. Data from interviews will be analyzed and the research findings will be discussed in my thesis. The raw data will be destroyed two years after the completion of the project, approximately on or before $31^{\text {st }}$ March 2021 . After the completion of the degree or earlier, the thesis or parts thereof might be published in journal articles or at conferences.

I assure confidentiality of all participants. Participants will only be revealed by pseudonyms. Your name and identity will remain confidential and will not be used in the thesis.

If you have any questions about the project, please feel free to contact:

Student:

Undhan Sevisari

sevisaundh@myvuw.ac.nz (+64) 221296503
Supervisor:

Dr. Ina Reichenberger

ina.reichenberger@vuw.ac.nz (+64) 44635375

This project has been review and approved by the Victoria University of Wellington, Pipitea Ethics Committee (HEC).

Convener:

judith.loveridge@vuw.ac.nz

(+64) 44636028 


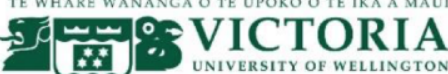 \\ Interaksi sosial di Situs Sharing Akomodasi Tak Berbayar: Motivasi Host dan Kolaborasi dalam menciptakan manfaat bersama}

\section{Informasi bagi peserta}

Halo,

Terima kasih atas ketertarikan saudara dalam project ini. Nama saya Undhan Sevisari dan saat ini saya sedang mengambil master of Tourism Management di Sekolah Management, Victoria University of Wellington, New Zealand. Saya sedang dalam tahapan menyelesaikan thesis saya dalam rangka merampungkan studi saya. Oleh karena itu, saya mengundang Saudara untuk ambil bagian dalam project riset saya. Berikut adalah informasi terkait dengan riset saya, mohon dapat dibaca dengan seksama sebelum memutuskan untuk berpartisipasi. Jika Saudara memutuskan untuk ambil bagian dalam riset ini, saya sangat berterima kasih dan mohon untuk menandatangani form kesediaan berpartisipasi. Namun, jika Saudara memutuskan untuk tidak mengambil bagian, saya juga berterimakasih sudah mempertimbangakan undangan berpartisipasi dari saya.

\section{Tujuan Riset Project:}

Thesis saya adalah tentang interaksi social antara host dan guest di akomodasi sharing tidak berbayar (CouchSurfing) di Indonesia. Saya ingin tahu apa yang menjadi motive dari host tersebut untuk ambil bagian dalam aktifitas CouchSurfing. Lebih lanjut, saya mempelajari bagaimana interaksi social tersebut menciptakan manfaat bagi host.

\section{Bagaimana saudara dapat membantu?}

Berpartisipasi dalam project ini berarti saudara bersedia untuk diwawancara dengan researcher (saya sendiri) dalam waktu dan tempat yang nyaman bagi anda. Interview akan direkam dan berlangsung kira-kira 30-45 menit. Namun, wawancara ini hanya akan dimulai atas persetujuan anda. Anda diperbolehkan untuk menolak untuk menjawab pertanyaan tertentu dan anda juga berhak untuk mengundurkan diri dalam project ini hingga Monday, 17 September 2018 baik melalui telefon, email maupun surat. Jika ini terjadi, semua informasi yang anda berikan akan dihapus.

\section{Apa yang akan terjadi dengan informasi yang anda berikan?}

Rekaman interview dan transcript akan disimpan dengan aman di computer yang sudah dilengkapi dengan password, yang hanya bisa diakses oleh saya dan supervisor saya. Data dari interview akan dianalisis dan hasil riset akan dibahas dalam thesis saya. Data mentah hasil interview akan dihapus setelah dua tahun saya menyelesaikan studi saya, kita-kira pada atau sebelum $31^{\text {st }}$ March 2021. Setelah atau sebelum gelar saya selesai, thesis saya atau bagian dari thesis tersebut akan diterbitkan di jurnal artikel atau di konferensi.

Saya memastikan kerahasiaan partisipan dalam riset ini. Partisipan akan dirujuk melalui nama samaran. Nama asli dan identitas lainnya akan dijamin kerahasiaanya dan tidak akan dipublikasikan dalam thesis.

Jika saudara memiliki pertanyaan tentang project ini, mohon untuk dapat disampaikan kepada saya atau supervisor saya berikut:

\author{
Mahasiswa: \\ Undhan Sevisari \\ sevisaundh@myvuw.ac.nz \\ (+64) 221296503
}

Supervisor:

Dr. Ina Reichenberger

ina.reichenberger@vuw.ac.nz

(+64) 44635375

Project ini telah mendapatkan review dan disetujui oleh Victoria University of Wellington, Pipitea Ethics Committee (HEC)/ Komite Etik Pipitea.

Yang berwenang:

judith.loveridge@vuw.ac.nz - (+64) 44636028 


\title{
Appendix B: Information sheet for Focus Group Discussion
}

\author{
TE WHARE WÃNANGA O TE ÖPOKO O TE IKA A MÃUI \\ T \\ Social Interaction in Non-Commercial Accommodation Sharing: Host's Motivation and Value Co-Creation
}

\section{INFORMATION SHEET FOR FOCUS GROUP}

You are invited to take part for Focus Group on Monday, $13^{\text {th }}$ August 2018, place to be advised by the Yogyakarta CouchSurfing community coordinator. Please read this information before deciding whether or not to take part. If you decide to participate, thank you. If you decide not to participate, thank you for considering this request.

Who am I?

My name is Undhan Sevisari and I am a Master student in School of Management at Victoria University of Wellington. This research project is work towards my Thesis.

\section{What is the aim of the project?}

As part of my thesis project, I am looking at interactions among host and guest in non-commercial's accommodation in Indonesia. Further, I am examining the motive of the host participating in CouchSurfing activities and how these interactions between host and guest generate benefits for hosts.

The Victoria University of Wellington Human Ethics Committee has approved this research with application reference number 0000026085 .

How can you help?

You have been invited to participate because you are part of CouchSurfing community and your opinion regarding this community will provide valuable inputs to my research. If you agree to take part you will be part of a focus group in Yogyakarta CouchSurfing community. I will ask you and other participants question about activities within CouchSurfing community and the importance of the community to its members. The focus group will take circa 30-45 minutes. I will audio record the focus group with your permission and write it up later.

The information shared during the focus group is confidential. That means after the focus group, you may not communicate to anyone, including family members and close friends, any details about the focus group. You can withdraw from the focus group at any time before the focus group begins. You can also withdraw while the focus group it is in progress. However it will not be possible to withdraw the information you have provided up to that point, as it will be part of a discussion with other participants.

\section{What will happen to the information you give?}

This research is confidential. This means that the researcher named below will be aware of your identity but the research data will be combined and your identity will not be revealed in any reports, presentations, or public documentation.

Only my supervisors and I will read the notes or transcript of the focus group. The focus group transcripts, summaries and any recordings will be kept securely and destroyed on 31th March 2021.

\section{What will the project produce?}

The information from my research will be used in Masters and after the completion of the degree or earlier, the thesis or parts thereof might be published in journal articles or at a conferences.

\section{If you accept this invitation, what are your rights as a research participant?}

You do not have to accept this invitation if you don't want to. If you do decide to participate, you have the right to:

- $\quad$ Choose not to answer any question;

- $\quad$ Ask for the recorder to be turned off at any time during the focus group;

- Withdraw from the focus group while it is taking part however it will not be possible to withdraw the information you have provided up to that point;

- $\quad$ Ask any questions about the study at any time;

If you have any questions or problems, whom can you contact?

If you have any questions, either now or in the future, please feel free to contact:

\section{Student:}

Name: Undhan Sevisari
Supervisor:

Name: Ina Reichenberger 


\section{TE WHARE WĀNANGA O TE ÜPOKO O TE IKA A MĀUI ITOMICTORIA

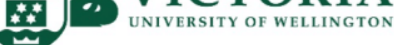 \\ Interaksi sosial di Situs Sharing Akomodasi Tak Berbayar: Motivasi Host dan Kolaborasi dalam menciptakan manfaat bersama}

\section{INFORMATION SHEET FOR FOCUS GROUP}

Halo,

Dengan ini saya mengundang anda untuk ambil bagian dalam focus group pada Senin, 13 Agustus 2018, tempat akan diupdate kemudian. Mohon dapat membaca informasi ini dengan seksama sebelum memutuskan untuk berpartisipasi. Jika anda memutuskan untuk berpartisipasi, saya berterima kasih. Namun, jika anda memutuskan untuk tidak ambil bagian, terima kasih telah mempertimbangkan undangan saya.

\section{Tentang saya:}

Nama saya Undhan Sevisari dan saya adalah mahasiswa master of tourism di School of Management di Victoria University of Wellington. Riset ini dalam rangka untuk menyelesaikan thesis saya.

\section{Tujuan riset ini:}

Thesis saya adalah tentang interaksi social antara host dan guest di akomodasi sharing tidak berbayar (CouchSurfing) di Indonesia. Saya ingin tahu apa yang menjadi motive dari host tersebut untuk ambil bagian dalam aktifitas CouchSurfing. Lebih lanjut, saya mempelajari bagaimana interaksi social tersebut menciptakan manfaat bagi host.

The Victoria University of Wellington, Human Ethics Committee telah menyetujui riset ini dengan nomor aplikasi 0000026085 .

\section{Bagaimana anda bisa membantu?}

Anda telah terpilih untuk berpartisipasi karena anda adalah bagian dari komunitas CouchSurfing dan opini anda terkait komunitas CouchSurfing akan memberikan input berharga terhadap riset saya. Jika anda bersedia untuk ambil bagian, saya akan bertanya tentang aktifitas dalam CouchSurfing community dan pentingnya komunitas ini bagi anggotanya. Focus group ini akan berlangsung kurang lebih 30-45 menit. Jika anda berkenan, saya akan merekam percakapan ini dan membuat transcript.

\section{What will happen to the information you give?}

Riset ini bersifat rahasia. Artinya, saya sebagai peneliti akan menjaga identitas anda dan identitas anda tidak akan dipublikasikan dalam laporan, presentasi maupun dokumentasi. Akses hanya diberikan kepada saya dan supervisor saya untuk membaca catatan maupun transcript diskusi. Transcrip, summary maupun rekaman akan disimpan dengan aman dan dihapus pada 31 Maret 2021.

\section{Bagaimana Prosedur Riset?}

Informasi dari riset saya akan digunakan dalam thesis dan setelah saya menyelesaikan gelar, thesis saya akan dipublikasikan dalam journal atau dipresentasikan dalam konferensi.

\section{Hak anda sebagai participant:}

Jika anda bersedia untuk berpartisipasi, anda memiliki hak untuk:

- $\quad$ Memilih untuk tidak menjawab pertanyaan;

- Meminta recorder untuk dimatikan ketika sedang melakukan diskusi;

- Menarik diri dari focus group selama berlangsung, namun anda tidak bisa menarik informasi yang anda berikan;

- Menanyakan tentang riset

Jika ingin mengetahui lebih lanjut tentang riset ini, anda dapat menghubungi: 


\section{Appendix C: Consent Form}

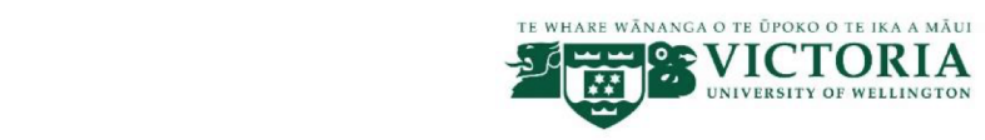

Social Interaction in Non-Commercial Accommodation Sharing: Host's Motivation and Value CoCreation

\section{Consent form}

I have read the Information Sheet concerning this student project and understand what it is about. All my questions have been answered to my satisfaction.

I know that:

- My participation in this project is entirely voluntary

- In the event that the line of questioning during the interview develops in such a way that I feel hesitant or uncomfortable I may decline to answer any particular question [s] and/ or may withdraw from the project immediately. As a consequence, my contribution to the study will immediately be destroyed.

- I may withdraw my contribution to the project until Monday, $17^{\text {th }}$ September 2018, either by phone, email or mail. As a consequence, my contribution to the study will be destroyed immediately.

- The interview is expected to last circa $30-45$ minutes and it is being electronically recorded.

- All recordings, notes and transcripts will be destroyed two years after the completion of the study approximately in $31^{\text {st }}$ March 2021 .

If you would like a copy of interview summary [please tick box as required]:

口 an interview summary to be sent to my email address

Email:

$\square \quad$ an interview summary to be sent to my postal address

Postal address:

You may provide feedback to interview's summary and send it through email address provided in the information sheet before Monday, $17^{\text {th }}$ September 2018

I agree to take part in this project. 


\section{TE WHARE WÃNANGA O TE ŨPOKO O TE IKA A MĀUI

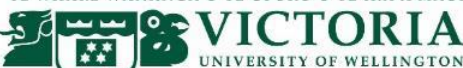 UNIVERSITY OF WELLINGTON \\ Interaksi sosial di Situs Sharing Akomodasi Tak Berbayar: Motivasi Host dan Kolaborasi dalam menciptakan manfaat bersama \\ Pernyataan Kesediaan Berpartisipasi}

Dengan ini, saya telah membaca informasi terkait dengan project mahasiswa yang bersangkutan dan saya telah mengerti dan memahami tujuan dari project tersebut. Saya telah memperoleh jawaban yang memuaskan atas informasi terkait project tersebut.

Saya mengerti bahwa:

- Partisipasi saya dalam project ini bersifat sukarela.

- Jika dalam interview saya merasa ragu atau saya merasa tidak nyaman dalam pertanyaan yang diajukan, saya berhak untuk menolak menjawab pertanyaan tertentu dan/ atau menarik diri dari project sesegera mungkin. Sebagai konsekuensinya, kontribusi saya dalam project ini akan segera dihapuskan/ ditiadakan.

- Saya bisa untuk menarik kontribusi saya dalam project ini hingga Senin, 17 September 2018, baik melalui telefon, email atau surat. Sebagai konsekuensinya, kotribusi saya terhadap project ini akan segera dihapuskan atau ditiadakan.

- Interview akan berlangsung sekitar 30 - 45 menit dan akan direkam secara elektronik.

- Semua rekaman, catatan maupun transcripts wawancara akan dihapus dalam kurun waktu dua tahun setelah yang bersangkutan menyelesaikan study-nya, kira-kira pada $31^{\text {st }}$ March 2021.

Saya mengharapkan [mohon centang yang diperlukan]:

Ringkasan ringkasan interview agar dikirim ke alamat email.

Email:

Ringkasan ringkasan interview agar dikirim ke alamat berikut.

Alamat:

Anda diperbolehkan memberikan feedback terhadap ringkasan interview dan mengirimkannya melalui alamat e-mail yang tersedia di lembaran informasi sebelum Senin, 17 September 2018.

Saya bersedia untuk berpartisipasi dalam project ini:

[Nama participant]
[Tandatangan participant]

[Tanggal] 


\title{
Appendix D: Consent Form for Focus Group
}

\author{
TE WHARE WĀNANGA O TE ŨPOKO O TE IKA A MĀUI \\ S⿹𠃌丨 \\ Social Interaction in Non-Commercial Accommodation Sharing: Host's Motivation and Value Co- \\ Creation
}

\section{CONSENT TO PARTICIPATE IN FOCUS GROUP}

This consent form will be held for 2 years.

Researcher: $\quad$ Undhan Sevisari, School of Management, Victoria University of Wellington.

I have read the Information Sheet and the project has been explained to me. My questions have been answered to my satisfaction. I understand that I can ask further questions at any time.

I agree to take part in a video recorded focus group.

understand that:

I acknowledge that I am agreeing to keep the information shared during the focus group confidential. I am aware that after the focus group, I must not communicate to anyone, including family members and close friends, any details about the focus group.

I can withdraw from the focus group while it is in progress however it will not be possible to withdraw the information I have provided up to that point as it will be part of a discussion with other participants

Any information I provide will be kept confidential to the researcher and the supervisor and will be destroyed in $31^{\text {st }}$ March 2021.

I understand that the results will be used for a Masters Thesis and/or academic publications and/or presented to conferences.

My identity will not be used in reports, nor will any information that would identify me.

Signature of participant:

Name of participant:

Date:

Contact details: 
政

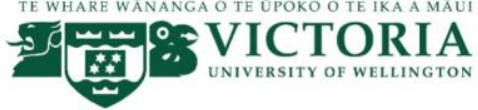

Social Interaction in Non-Commercial Accommodation Sharing: Host's Motivation and Value CoCreation

\section{Pernyataan kesedian untuk berpasrtisipasi dalam focus group}

Dokumen ini akan disimpan selama 2 tahun

Peneliti: Undhan Sevisari, School of Management, Victoria University of Wellington.

Saya telah membaca lembar informasi dan saya telah mendapatkan penjelasan tentang project ini. Pertanyaan saya telah dijawab. Saya mengerti bahwa saya dapat bertanya sewaktu-waktu.

Saya bersedia untuk ambil bagian dalam focus group ini

xya mengerti bahwa:

Saya menyetujui untuk menjaga kerahasiaan informasi selama focus group. Saya menyadari bahwa setelah focus group, saya tidak akan mengkomunikasikan dengan siapapun, termasuk anggota keluarga dan teman dekat, detil pelaksanaan focus group.

Saya dapat menarik diri dari focus group ketika focus group sedang berlangsung, namun saya mengetahui bahwa saya tidak bisa menarik informasi yang telah saya berikan karena hal tersebut telah menjadi bagian dari diskusi dengan participant lain.

Segala informasi yang saya berikan bersifat rahasia. Akses hanya diberikan kepada peneliti dan supervisor. Informasi yang saya berikan akan dihapus pada 31 Maret 2021.

Saya mengerti bahwa hasil riset ini akan digunakan untuk master thesis dan/ atau publikasi akademik dan/ atau dipresentasikan dalam konferensi.

Identitas saya tidak akan digunakan dalam laporan, dan tidak akan ada informasi yang menggunakan identitas saya.

Tandatangan participant:

Nama participant:

Tanggal:

Contact details: 


\section{Appendix E: Interview Guidelines}

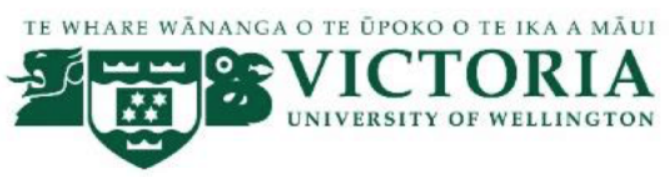

Social Interaction in Non-Commercial Accommodation: Host's Motives and Value Co-Creation

\section{Interview Guidelines}

\section{Introduction}

- Personal introductions

- Thank you for taking the time

- Research purpose: Through this interview, I am looking for the motivations of hosts participate in CouchSurfing. Further, I am exploring the reason for giving free accommodation to strangers. Next, I am interested to know how is your relationship with guest and how you interact with them during their stay. Lastly, I am also interested to know how important is the interaction to you.

- Confirmation of agreement to information sheet

\section{Question on CouchSurfing}

- How long have you been a CouchSurfing member?

- Why are you a CouchSurfing member? Prompt:

- What does it benefit you?

- Have you had any experience as guest in CouchSurfing?

- How does it feel to you to be a guest in someone else's house?

- Why do you want to give free accommodation? What does it mean to you? Prompt:

- Do you have expectation toward guest?

\section{Social Interaction in CouchSurfing}

- Can you describe yourself as a host?

- Could you tell me your latest hosting experience in CouchSurfing? Prompt:

- What do you usually do when the guest arrive/ during their stay?

- Can you share some memorable experience with guest during their stay?

- Can you share any experience that you do not perceive in such a positive way?

\section{Conclusion}

- Anything else you would like to add?

- Thank you for taking the time

- Explaining further research process. How their data will be analyzed and how they can withdraw and provide feedback to the interview's summary 


\section{Interview Guidelines}

\section{Pengenalan}

- Perkenalan diri

- Terima kasih atas waktunya

- Tujuan riset: melalui interview ini, saya tertarik untuk mempelajari motivasi host berpartisipasi in CouchSurfing. Lebih jauh, saya ingin mempelajari alasan host memberikan akomodasi gratis kepada orang asing. Selanjutnya, saya tertarik untuk mengetahui bagaimana interaksi dan hubungan host dengan tamu selama mereka menginap. Terakhir, saya tertarik untuk mengetahui bagaimana pentingnya interaksi ini untuk host.

- Konfirmasi persetujuan atas lembar informasi.

\section{Pertanyaan tentang CouchSurfing}

- Berapa lama kamu telah menjadi CouchSurfing member?

- Mengapa kamu menjadi CouchSurfing member? Prompt:

- Apakah manfaatnya untuk anda?

- Pernahkah anda memiliki pengalaman sebagai tamu di CouchSurfing?

- Bagaimana rasanya menjadi tamu di rumah orang lain?

- Mengapa kamu memberikan akomodasi gratis? Apa artinya untuk anda? Prompt:

- Kamu punya harapan terhadap tamu?

\section{Interaksi social di CouchSurfing}

- Bagaimana kamu melihat diri kamu sebagai seorang host?

- Bisakah anda ceritakan pengalaman sebagai host terakhir di CouchSurfing? Prompt:

- Apa yang biasanya kamu lakukan ketika tamu datang/ selama mereka stay?

- Bisakah anda menceritakan beberapa pengalaman yang berkesan dengan tamu ketika mereka tinggal?

- Bisakah anda menceritakan pengalaman yang anda anggap kurang positive?

\section{Kesimpulan}

- Ada hal lain yang ingin kamu tambah?

- Terima kasih atas waktunya

Jelaskan tentang proses riset. Bagaimana data mereka akan dianlisis dan bagaimana mereka dapat menarik data dan memberikan feedback terhadap summary of the interview. 


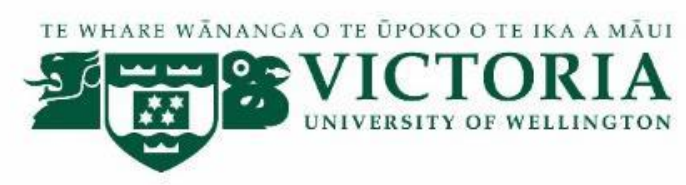

Social Interaction in Non-Commercial Accommodation: Host's Motives and Value Co-Creation

\section{Interview Guidelines for Focus Group}

\section{Introduction}

- Personal introductions

- Thank you for taking the time

- Research purpose: Through this interview, I am looking for the motivations of hosts participate in CouchSurfing. Further, I am exploring the reason for giving free accommodation to strangers. Next, I am interested to know how social interaction between host and guest occur in CouchSurfing and what values host's derive from the interaction.

- Confirmation of agreement to information sheet

\section{General Question on CouchSurfing}

- Why are you a CouchSurfing member?

- How important is this membership to you?

- Could you tell me, how does this CouchSurfing community established?

- Please describe the activities within the CouchSurfing community? Prompt:

If the participant mentions sharing: what does sharing mean to you? What do you share?

- Do you see any benefits from these activities?

- In which role do you see yourself in the community as member of CouchSurfing?

\section{Conclusion}

- $\quad$ Anything else you would like to add?

- Thank you for taking the time

- Explaining further research process. How their data will be analyzed and how 
Social Interaction in Non-Commercial Accommodation: Host's Motives and Value Co-Creation

\section{Interview Guidelines for Focus Group}

\section{Perkenalan}

- Personal introductions

- Thank you for taking the time

- Research purpose: Through this interview, I am looking for the motivations of hosts participate in CouchSurfing. Further, I am exploring the reason for giving free accommodation to strangers. Next, I am interested to know how social interaction between host and guest occur in CouchSurfing and what values host's derive from the interaction.

- Confirmation of agreement to information sheet

\section{General Question on CouchSurfing}

- Why are you a CouchSurfing member?

- How important is this membership to you?

- Could you tell me, how does this CouchSurfing community established?

- Please describe the activities within the CouchSurfing community? Prompt: If the participant mentions sharing: what does sharing mean to you? What do you share?

- Do you see any benefits from these activities?

- In which role do you see yourself in the community as member of CouchSurfing?

\section{Conclusion}

- Anything else you would like to add?

- Thank you for taking the time 